\title{
Designing Biopolymer Microgels to Encapsulate, Protect and Deliver Bioactive Components: Physicochemical Aspects
}

\section{David Julian McClements}

Department of Food Science, University of Massachusetts, Amherst, MA 01003

Journal: Advances in Colloid and Interface Science

Submitted:

Revised:

Contact Information: David Julian McClements, Department of Food Science, University of Massachusetts Amherst, Amherst, MA 01003, USA. mcclements@foodsci.umass.edu; Tel: 4135451019. 


\begin{abstract}
Biopolymer microgels have considerable potential for their ability to encapsulate, protect, and release bioactive components. Biopolymer microgels are small particles (typically $100 \mathrm{~nm}$ to $1000 \mu \mathrm{m}$ ) whose interior consists of a three-dimensional network of cross-linked biopolymer molecules that traps a considerable amount of solvent. This type of particle is also sometimes referred to as a nanogel, hydrogel bead, biopolymer particles, or microsphere. Biopolymer microgels are typically prepared using a two-step process involving particle formation and particle gelation. This article reviews the major constituents and fabrication methods that can be used to prepare microgels, highlighting their advantages and disadvantages. It then provides an overview of the most important characteristics of microgel particles (such as size, shape, structure, composition, and electrical properties), and describes how these parameters can be manipulated to control the physicochemical properties and functional attributes of microgel suspensions (such as appearance, stability, rheology, and release profiles). Finally, recent examples of the utilization of biopolymer microgels to encapsulate, protect, or release bioactive agents, such as pharmaceuticals, nutraceuticals, enzymes, flavors, and probiotics is given.
\end{abstract}

Keywords: microgels; hydrogel beads; biopolymer particles; encapsulation; release 


\section{Introduction}

There is considerable interest in the oral delivery of bioactive agents in the food, supplement, and pharmaceutical industries [1-6]. These bioactive agents include a diverse range of materials, including pharmaceuticals, nutraceuticals, vitamins, minerals, macronutrients, antimicrobials, probiotics, and enzymes. There are numerous challenges associated with incorporating these materials into many commercial products intended for oral ingestion, such as poor dispersibility or stability characteristics, or the need for a particular release or activity profile [7]. Many bioactive agents are physically or chemically unstable and may undergo undesirable changes in the product or within the gastrointestinal tract (GIT) that negatively impact their bioavailability and bioactivity. Enzymes may lose their activity when solution or environmental conditions are altered e.g., $\mathrm{pH}$, ionic strength, solvent quality, or temperature [8]. Polyunsaturated lipids may undergo chemical degradation due to oxidation when exposed to high oxygen levels, elevated temperatures, light, or pro-oxidants $[9,10]$. Probiotics may lose viability during storage within a functional food product, or during their passage through the GIT $[11,12]$. Many of the challenges associated with incorporating bioactive agents into foods, supplements, and pharmaceutical products can be overcome using well designed delivery systems $[7,13]$. In this manuscript, the focus is on the design, fabrication, and application of biopolymer microgels (also sometimes referred to as biopolymer particles, nanogels, hydrogel beads, or microspheres) as oral delivery systems for bioactive agents. This type of microgel consists of relatively small $(0.1$ to $1000 \mu \mathrm{m})$ particles whose interior is comprised of a network of cross-linked biopolymer molecules that traps a considerable amount of solvent (usually water) [14]. Microgels can easily be prepared from food-grade ingredients (such as proteins and polysaccharides) using simple processing operations (such as mixing and injection) and are therefore particularly suitable for this type of application [14-18]. A brief overview of progress in the utilization of microgels for encapsulation, protection, and delivery of nutraceuticals has recently been published [19]. The present article provides a more comprehensive review of the fabrication, characteristics, physicochemical properties, and applications of biopolymer microgels intended for oral ingestion, with particular emphasis on food-grade systems. 


\section{Microgel Constituents}

The constituents used to form biopolymer microgels have a major impact on their functional attributes [20]. The most common materials used to fabricate food-grade microgels intended for oral ingestion are proteins and polysaccharides. Different types of biopolymer molecules vary considerably in their molecular and physicochemical properties, such as molar mass, conformation, branching, electrical characteristics, polarity, solubility, viscosity enhancement, gelation mechanism, hydrogel properties, and surface activity. Even within a particular class of biopolymers (such as pectin), there are considerable variations in molecular and physicochemical properties depending on its source, as well as the isolation, purification, and processing methods used.

Consequently, it is always important to select the most appropriate biopolymer ingredient for a particular application.

The properties of the different types of biopolymers that can be utilized to form microgel particles have been reviewed extensively elsewhere [7, 21-23]. For this reason, only a brief summary of their molecular, physicochemical, and functional attributes are given here (Table 1). Some of the most important physicochemical properties to consider when selecting an appropriate biopolymer are summarized below:

- Solubility characteristics: The range of solution conditions where the biopolymer molecules are soluble in aqueous solutions should be known, e.g., $\mathrm{pH}$, ionic strength, solvent type, and temperature. This is important because the biopolymer molecules must frequently be dissolved in aqueous solutions before they can be utilized to form microgels. For example, some polysaccharides (such as agar) and proteins (such as gelatin) have to be heated above their helix-coil transition temperature to breakdown residual helical structures before they can be used to form hydrogels through a cold-set mechanism [21]. Moreover, the low solubility of biopolymers in specific solvents can be utilized to form microgels, e.g., the formation of zein nanoparticles by the antisolvent precipitation method [24, 25].

- Gelling mechanism: The factors that impact the gelation of a solution of biopolymer molecules should be thoroughly understood when designing an optimum method of producing microgels [21]. For example, the critical temperature that a solution must be heated above or cooled below to induce 
gelation/melting of heat-set biopolymers (such as whey or soy protein) or cold-set biopolymers (such as agar, carrageenan, or gelatin) should be known. Similarly, the critical mineral ion concentration where a sol-gel transition occurs for biopolymers that undergo ionic gelation should be known, e.g., the critical calcium concentration for alginate [26] or the critical potassium concentration of carrageenan [27].

- Electrical properties: The change in surface potential ( $\zeta$-potential) of the biopolymer molecules with $\mathrm{pH}$ should be known because this often influences their ability to form microgels, e.g., by cross-linking with oppositely charged mineral ions or biopolymer molecules [28]. Changes in the internal electrical properties of microgels (such as the charge density and sign) can also be used to control the retention and release of charged bioactive molecules [29]. Moreover, the $\zeta$-potential versus $\mathrm{pH}$ profile of the microgels themselves influences their aggregation stability by altering the electrostatic interactions between them [30], as well as their interactions with other charge species. For example, charged microgels can be coated with oppositely charged polyelectrolytes using electrostatic deposition [31].

- Degradability: Knowledge of the factors that impact the degradation of the biopolymer chains may be important in designing microgels with specific release properties or biodegradability. Many proteins, lipids, and starches are degraded in the upper GIT due to the presence of proteases, lipases, and amylases in the gastrointestinal fluids [32,33]. Conversely, many polysaccharides (dietary fibers) are indigestible in the upper GIT, but are degraded by enzymes released by bacteria in the colon [34]. This information can be used to create microgels that release their payloads in particular regions of the GIT, such as the mouth, stomach, small intestine, or colon [35].

- Practical Factors: There are a number of economic, regulatory, and consumer factors that should be considered when selecting an appropriate biopolymer for a particular microgel application. For example, the cost-in-use, reliability of supply, and label-friendliness (e.g., vegetarian, vegan, or Kosher) may have to be considered. 
Selection of the most appropriate biopolymer or combination of biopolymers to fabricate a microgel suspension is one of the most critical decisions that must be made, and will largely determine the potential success of the resulting encapsulation system. Ideally, the molecular characteristics and physicochemical properties of the particular biopolymer ingredients used should be determined, because there are appreciable variations from batch to batch.

\section{Microgel Fabrication}

The fabrication of microgels loaded with bioactive agents usually involves two steps that may occur sequentially or consecutively: particle formation and particle gelation (Figure 1). In the particle formation step, an aqueous solution containing bioactive agents and biopolymer molecules is converted into small biopolymer-rich particles (typically between about $100 \mathrm{~nm}$ and $1 \mathrm{~mm}$ in diameter). Hydrophilic bioactives are usually directly dissolved in this aqueous solution, whereas hydrophobic bioactives first have to be dissolved within lipid droplets or other hydrophobic vehicles (Figure 2). In the particle-gelation step, the small biopolymer-rich particles are converted into a microgel by altering solution or environmental conditions to cross-link the biopolymer chains. The particle formation and gelation methods utilized to form a particular type of microgel depend on the nature of the constituents and fabrication method used [7, 15]. In general, fabrication methods rely on a combination of physiochemical phenomenon (such as molecular association, cross-linking, or phase separation) and mechanical processes (such as extrusion, atomization, or shearing). A number of the most common methods of forming microgels are briefly discussed below, and more details can be found in other recent reviews $[7,15,16,36-38]$.

\subsection{Injection Methods}

A common feature of this group of methods is that the particles are formed by passing an aqueous solution containing bioactive agents and biopolymers through a narrow orifice into a water-immiscible medium (such as air or oil). This process may lead to biopolymer-rich particles being formed one at a time, or multiple particles being 
formed simultaneously. After the particle formation step, the biopolymers are crosslinked using a suitable method to produce microgels.

\subsubsection{Simple Extrusion Methods}

In this method, an aqueous solution containing a mixture of bioactives and biopolymers is first prepared, and then injected into a gas phase. The particles formed by simple extrusion methods typically have a roughly spherical shape because of the relatively high surface tension at the air-water interface. Particle-gelation may occur after the biopolymer-rich particles travel through the gas phase or after they come into contact with a gelling solution (Figure 3).

Particle-gelation may occur within the gas phase due to a change in temperature or due to solvent evaporation $[39,40]$. Some biopolymers form cross-links when they are cooled below a critical temperature (such as gelatin) whereas others form cross-links when they are heated above a critical temperature (such as many globular proteins). Thus microgel particles can be formed by cold- or hot-gelation by controlling the temperature of the gas phase that the particles are injected into. Conversely, particle-gelation may occur within the gas phase due to evaporation of the water [41]. The removal of water from the particles increases the concentration of biopolymer molecules within them, as well as any other components in the aqueous phase (such as acids, bases, minerals, or cross-linking agents). In this case, the system is designed to remain in a liquid state at high water contents, but gel when the water content decreases below a critical value. The resulting microgels can then be collected and used as is or they can be dispersed within an aqueous solution [39].

Particle-gelation can also be induced by directing the liquid particles into an aqueous gelling solution containing a cross-linking agent for the biopolymer chains, such as specific mineral ions, acids/bases, or enzymes [15]. For example, multivalent cations (such as calcium and magnesium) or monovalent cations (such as sodium or potassium) have been included in gelling solutions to cross-link negatively charged polysaccharides (such as alginate, carrageenan, or pectin) and proteins (above their isoelectric point). Specific chemicals (such as gluteraldehyde or genipin) or enzymes (such as transglutaminase or laccase) can also be included in gelling solutions to cross link biopolymers with appropriate functional groups [42, 43]. For some biopolymers, 
controlling the temperature of the gelling solution can induce physical cross-linking. The formation of gelatin microgel particles is an example of cold-gelation. In this case, the microgel particles are formed by injecting liquid particles containing a hot-gelatin solution into a cold environment [44]. Below their helix-coil transition temperature, the gelatin molecules form physical cross-links due to hydrogen bonding, which leads to particle gelation and microgel formation. The formation of globular protein microgel particles is an example of hot-gelation. In this case, the microgel particles are formed by injecting liquid particles containing a solution containing globular proteins (e.g., whey, egg, or soy proteins) into a hot environment $[45,46]$. The native globular proteins unfold above their thermal denaturation temperature $\left(\mathrm{T}_{\mathrm{m}}\right)$, which promotes aggregation with their neighbors due to strong hydrophobic attraction and disulfide bond formation. It should be noted that this approach may not be suitable for thermally labile bioactives, such as probiotics, enzymes, or some nutraceuticals.

Rather than injecting the solution of bioactives and biopolymers into a gas phase, it is also possible to directly inject it into an oil phase [39]. Approximately spherical biopolymer-rich particles are formed at the tip of the nozzle because of the relatively high oil-water interfacial tension. These particles can then be gelled by altering the temperature (heating or cooling) or by including cross-linking agents in the oil phase (such as organic forms of acids, bases, or multivalent ions). This method has many similarities to the emulsion-templating method discussed later. The main disadvantage of this method is that the oil phase must be removed after the microgels are formed, which typically involves the use of an organic solvent. On the other hand, relatively small droplets with well-defined particle sizes can be produced using this method, which is a major advantage for certain applications.

Including different components within the biopolymer solution prior to injection enables one to vary the internal composition of the microgels produced using the simple extrusion method. For example, one could include non-gelling biopolymers, lipid droplets, antioxidants, and buffering agents to control the retention, protection, and release of bioactives. The diameter of the nozzle used to inject the bioactive/biopolymer solution mainly determines the dimensions of the microgels produced using this method. Core-shell structures can be formed using simple extrusion methods by utilizing a co- 
axial nozzle with one type of gelling biopolymer solution on the inside and another on the outside [47].

One of the most common applications of the simple extrusion method is to fabricate calcium alginate beads containing bioactive substances. This approach has been used to control the rate of lipid digestion and bioactive release under simulated small intestine conditions [48-50]. In addition, this type of microgel has also be used to encapsulate and protect a variety of bioactive components, including insulin [51], thyme oil [52], flavor molecules [53] and curcumin [54]. There is considerable scope for controlling the composition and structure of calcium alginate beads so as to tailor their functional properties for specific applications [55]. A similar method can be used to form microgels from other combinations of biopolymers and mineral ions, such as carrageenan and potassium [56].

\subsubsection{Atomization Methods}

Atomization methods typically generate a huge number of particles when the aqueous solution containing the bioactives and biopolymers is passed through the orifice, which is in contrast to simple extrusion methods that usually generate particles one at a time $[15,39,57]$. The most commonly used atomization methods for producing microgels are spray drying and spray chilling. Spray drying of a biopolymer solution typically leads to the formation of a powder that contains dehydrated biopolymer-rich particles. Conventionally, the powder particles produced by spray drying are designed to simply dissolve when they are added to an aqueous solution and thereby release the biopolymer molecules [57]. However, microgels can be formed using the spray drying method using a number of approaches. First, a preexisting microgel suspension can be converted into a powder using spray drying so that it will release microgels when reconstituted in water. Second, a solution of biopolymers can be converted into a powdered form, and then the powder particles can be dispersed into water containing a cross-linking agent, which leads to the formation of microgels provided that the gelation rate is higher than the dissolution rate [39]. Third, it is also possible to cross-link the biopolymer chains within the particles formed during the spray drying process, so that microgels are released when the powder particles are dispersed in water [58]. 
Multiple steps are associated with particle formation by the spray drying method [57]. First, the bioactives and biopolymers have to be dissolved or dispersed in a suitable solvent (water or organic solvent) that has a low enough viscosity to by pumped through a small nozzle. When the solution passes through the nozzle it is atomized, i.e., converted into a mist of multiple small biopolymer-rich drops. The nozzle is located inside a heated chamber, which causes the solvent to rapidly evaporate. The resulting solidified biopolymer-rich particles are then collected using a cyclone or filter bag. Thermally-sensitive bioactive agents can often survive this process because it occurs very quickly and because solvent evaporation is an endothermic process that inhibits excessive heating of the particles. Typically, the solid particles present in the powders produced by spray drying are somewhere in the range 10 to $100 \mu \mathrm{m}$ [57].

Spray drying is already widely used in the food and pharmaceutical industries and has been used to encapsulate numerous types of bioactive agents, including proteins, peptides, flavors, lipids, and probiotics $[57,59,60]$. The bioactive agents can be protected from degradation during storage by selecting appropriate wall materials. Typically, it is advisable to use carbohydrates or proteins that form a glass matrix in the dried form that inhibits the molecular diffusion of oxygen. The properties of the spray dried powder must also be carefully controlled to obtain desirable handling and reconstitution properties.

Spray chilling methods also involve the formation of multiple particles by passing a biopolymer solution through a nozzle, but in this case particle gelation is performed by cooling [40]. These methods are usually utilized to produce fat particles by spraying a liquid oil into a cold environment to convert the liquid oil droplets into solid fat particles. However, it may also be possible to utilize them to form microgels by spraying an aqueous solution containing the bioactives and biopolymers through a small nozzle into a cold chamber that promotes particle gelation. Cold-set biopolymers such as gelatin or agar could be used to form microgel suspensions using this approach. However, there are few examples of this approach being used for this purpose, and this could be an interesting area for future studies. 


\subsubsection{Microfluidic and Microchannel Methods}

Microgel particles with finely controlled diameters, compositions, and surface characteristics can be formed using microfluidic and microchannel devices [61-64]. Typically, an aqueous solution containing bioactives and biopolymers is injected into an oil phase to form the particles, and then the environmental conditions are altered to cross-link the biopolymers and gel the particles (Figure 4). These approaches are therefore somewhat similar to the simple injection methods discussed earlier, but specially designed devices with well-defined internal architectures are required to carry them out. These devices are typically manufactured from polymers using nanofabrication methods. Microfluidic and microchannel devices can be used to create microgels with specific compositions, dimensions, morphologies, and internal structures (e.g., dispersion or core-shell structures) by altering flow rates and channel properties (e.g., diameters, shapes, polarity, and architecture). A simple example of a microfluidic device that can be used to fabricate microgels is illustrated in Figure 4. An aqueous solution containing bioactives and biopolymers is forced through a narrow channel into a flowing oil phase, which leads to the formation of spherical biopolymer-rich particles due to interfacial tension effects. The biopolymers are then cross-linked by altering environmental conditions (such as temperature) or by adding an appropriate gelling agent (such as an organic acid, base, or salt). Microfluidic and microchannel devices are particularly suitable for research and development applications because of the close control over the characteristics of the microgels formed. Nevertheless, they are less suitable for widespread commercial applications because of difficulties associated with economically scaling the process up.

Microfluidic devices have recently been used to produce alginate nanogels and microgels with controllable particle compositions, dimensions, shapes, and pore sizes by varying fabrication parameters such as channel design, mixing time, flow rates, and solution compositions [65-67]. These devices can be used to produce microgels with well-defined diameters ranging from around $100 \mathrm{~nm}$ to $1000 \mu \mathrm{m}$ [65], and that can have a high degree of droplet monodispersity [68]. 


\subsubsection{High-Pressure Microfluidization Methods}

Recently, it has been shown that very small $(<100 \mathrm{~nm})$ microgel particles can be produced using a dual-channel high-pressure microfluidization method that involves impinging a stream of alginate into a stream of calcium ions at high velocity [69]. When the two fluid streams collide they generate intense turbulent forces that leads to the formation of small particles containing alginate chains cross-linked by calcium ions. This type of technique may be important for certain commercial applications where small microgel particles are desirable. This method can also be scaled up as there are commercial dual-channel microfluidization devices available.

\subsection{Templating Methods}

A common feature of this group of methods is that some form of template is used to create particles consisting of bioactive agents and biopolymers dispersed in an aqueous solution (particle formation), and then the environmental conditions are changed to induce cross-linking of the biopolymer chains (particle gelation) and form microgels [16, 55]. A potential advantage of templating methods is that they can produce microgels with well defined dimensions, shapes, and structures. However, many of them are currently only capable of producing small batches of samples, and are therefore more suitable for small-scale research studies than for large-scale commercial production of microgels.

\subsubsection{Emulsion-based Methods}

In this case, the particle-formation step involves creating a water-in-oil (W/O) emulsion by homogenizing an aqueous phase containing bioactive components and biopolymer molecules with an oil phase containing oil and a hydrophobic surfactant (Figure 5) [70, 71]. This process leads to the generation of biopolymer-rich water droplets that are dispersed in an oil phase. The particle-gelation step involves crosslinking the biopolymer molecules inside the water droplets by changing system conditions in an appropriate manner. For instance, the W/O emulsion could be cooled below a critical temperature to cross-link a cold-setting biopolymer (such as gelatin), or heated above a critical temperature to cross-link a heat-setting biopolymer (such as whey protein). An alternative approach is to incorporate a chemical cross-linking agent into the 
oil phase that will diffuse into the water droplets and cross-link the biopolymer chains, such as organic acids, bases, or minerals ions. After formation, the microgel particles can be isolated from the oil phase using centrifugation, filtration, or solvent extraction methods. The selection of a suitable oil-soluble surfactant to form the initial water droplets in the $\mathrm{W} / \mathrm{O}$ emulsion is an important consideration.

The composition of the microgel particles formed using this method can be varied by including different constituents in the aqueous phase used to prepare the initial water-inoil emulsion, such as non-gelling biopolymers, lipid droplets, antioxidants, or buffers. The dimensions of the microgel particles can be controlled by producing water droplets in the W/O emulsion with different particle size distributions, which can be achieved by using different homogenization methods, operating conditions, and emulsifiers. One of the major advantages of the inverse emulsion templating approach is that microgels can be produced with a range of different well-defined sizes (e.g., 0.1 to $1000 \mu \mathrm{m})$.

This approach has been used to encapsulate a variety of substances in microgels, including bioactive lipids in protein microgels [71-73], bioactive proteins in alginate microgels [74], an Alzheimer drug in alginate-CMC microgels [75, 76], and ibuprofen in alginate-locust bean gum microgels [77].

\subsubsection{Lithographic Methods}

Microgels with highly controlled dimensions and morphologies can be fabricated using lithographic methods $[20,62,78,79]$. An aqueous solution containing the bioactives and biopolymers is poured into a mold (template) that has a well-defined size and shape (particle formation), and then the environmental or solution conditions are altered to promote cross-linking of the biopolymer chains (particle gelation) (Figure 6). Altering the temperature (heating or cooling) or adding a gelling agent (such as an acid, base, mineral ion, or enzyme) are two of the most common methods of promoting the gelation of food biopolymers. After gelation, the microgels are removed from the mold, and can then be utilized in this form or they can be dispersed into an aqueous solution. Nanofabrication methods are typically used to create molds that contain multiple indentations with well-defined shapes and so it is possible to simultaneously produce multiple small uniform microgels. 
The utilization of this approach to fabricate microgels with different morphologies and compositions from food-grade biopolymers has been reported [80, 81]. For example, alginate microgels with different sizes (diameters ranging from to 10 to $400 \mu \mathrm{m}$ ) have been fabricated using templates with different dimensions [81]. This method is highly suitable for studying the major factors impacting the functional performance of microgel particles since particles with different sizes and morphologies can be prepared [82]. However, it is less suitable for large-scale fabrication of microgel particles on a commercial level due to difficulties in economic scale-up.

\subsection{Biopolymer Phase Separation Methods}

Mixtures of two or more different kinds of biopolymers in aqueous solutions may undergo phase separation and form two separate phases. These phase-separated systems can then be converted into "water-in-water" emulsions by applying mechanical forces (particle formation). Microgel beads can then be formed by changing environmental or solution conditions to promote cross-linking of the biopolymers in the internal water phase (particle gelation). The two most common approaches of forming microgels utilize either attractive biopolymer-biopolymer interactions (electrostatic complexation) or repulsive ones (thermodynamic incompatibility).

\subsubsection{Electrostatic Complexation}

In this case, particle-formation and particle-gelation usually occur simultaneously when solution conditions are altered due to an electrostatic attraction between two oppositely charged biopolymers [28, 46, 83, 84]. Typically, a solution is prepared that contains the bioactive agent and two types of biopolymers with different electrical characteristics ( $\zeta$-potential versus $\mathrm{pH}$ profiles). A number of alternatives are possible for the biopolymer pair selected: an anionic polysaccharide and cationic protein $(\mathrm{pH}<\mathrm{pI})$; a cationic polysaccharide and anionic protein $(\mathrm{pH}>\mathrm{pI})$; a cationic protein and anionic protein; a cationic polysaccharide and anionic polysaccharide. When a protein is one of the pair, the two biopolymers can be directly mixed together at the final $\mathrm{pH}$ where they have opposite charges, or they can be mixed together at a $\mathrm{pH}$ where they have similar charges and then adjusted to the final $\mathrm{pH}$ to induce complexation. The bioactive agents should associate with one or both of the biopolymer molecules in order to be 
encapsulated inside the microgel particles formed. Different kinds of electrostatic complexes may be formed from oppositely charged biopolymers depending on solution conditions, including soluble complexes, complex coacervates and precipitates [28]. Soluble complexes are relatively small clusters of biopolymers that remain readily dispersed in aqueous solutions. Complex coacervates are typically formed under conditions where soluble complexes associate with each other and form a separate biopolymer-rich phase. As mentioned earlier, mechanical forces can be applied to this phase separated system to form a W/W emulsion whose inner droplets can be gelled by changing environmental or solution conditions. Precipitates at densely packed clusters of biopolymers that normally sediment from solution, and are therefore typically less useful for encapsulation purposes.

One of the potential drawbacks of using electrostatic complexes is that their stability is highly sensitive to $\mathrm{pH}$ and ionic strength $[85,86]$. If the $\mathrm{pH}$ is adjusted to a value where the two biopolymers have similar charges, then they will tend to dissociate. Similarly, if the ionic strength is increased above a certain level the complexes tend to dissociate because of the weakening of the electrostatic attraction due to electrostatic screening effects. The $\mathrm{pH}$ and salt sensitivity of electrostatic complexes may be a problem in some applications, but in other applications it may be utilized to develop delivery systems that release bioactive agents in response to a $\mathrm{pH}$ or salt trigger. The stability of electrostatic complexes to dissociation can often be improved after they have been formed by carrying out an additional internal cross-linking step or by coating them with biopolymer shells [87]. Internal cross-linking of the biopolymers may be carried out using various processes, including adding chemical (such as gluteraldehyde), enzymatic (such as transgluteminase), or ionic (such as calcium) cross-linking agents or by altering the temperature to induce cold or hot gelation of one or both of the biopolymers. The coating of coacervates is often carried out by electrostatic deposition of biopolymers only oppositely charged electrostatic complexes.

Microgels formed by electrostatic complexation of biopolymers have been utilized to encapsulate and protect various types of bioactive agents, including vitamins (e.g., A, C, $\mathrm{D}$ and $\mathrm{K}$ ) [88-90], nutraceuticals (e.g., anthocyanins, astaxanthin, $\beta$-carotene, and curcumin) [91-94], proteins and peptides [95, 96], lipids [97, 98], and flavors [99-101]. 


\subsubsection{Thermodynamic incompatibility}

Microgels can also be formed from mixtures of biopolymers using a thermodynamic incompatibility method $[20,28,84,102]$. Unlike the electrostatic complexation method, which relies on attractive interactions between two or more biopolymers, the thermodynamic incompatibility method relies on repulsive interactions between biopolymers. An aqueous solution containing two biopolymers (A and B) that have a strong net repulsion between them (usually due to steric exclusion effects) may separate into two phases. One of the phases $\left(\mathrm{W}_{1}\right)$ is rich in biopolymer-A and depleted in biopolymer-B, while the other phase $\left(\mathrm{W}_{2}\right)$ is rich in biopolymer-B and depleted in biopolymer-A. This phase separated system can be converted into a W/W emulsion by applying mechanical forces, such as shearing. The nature of the W/W emulsion formed depends on the relative volume fraction of the $\mathrm{W}_{1}$ and $\mathrm{W}_{2}$ phases. When $\mathrm{W}_{1}>\mathrm{W}_{2}$, then a $\mathrm{W}_{2} / \mathrm{W}_{1}$ emulsion is formed, and vice versa. One of the main limitations of this type of system is that the dispersed phase particles are highly susceptible to coalescence, which eventually leads to phase separation. This problem can be overcome by cross-linking the dispersed phase particles after formation of the W/W emulsion. Cross-linking of the biopolymers can be achieved using physical, chemical, or enzymatic approaches as discussed for other systems [103]. Alternatively, the disperse phase particles can be stabilized against aggregation by covering them with a coating of different biopolymer molecules $[87,104]$. This can often be achieved by electrostatic deposition of an oppositely charged biopolymer onto the surfaces of the charged microgel particles.

As with other methods, hydrophilic bioactives can be directly mixed with the aqueous biopolymer solution before particle-formation, whereas hydrophobic bioactives can be trapped in oil droplets (or other hydrophobic particles) before mixing with the biopolymer solution used for particle formation. The phase separation approach has been used to form caseinate microgel particles containing $\omega$-3-rich fish oils and other lipids $[105,106]$.

\subsection{Antisolvent Precipitation}

Microgels can be formed based on the fact that some biopolymers have good solubility in one solvent, but poor solubility in another solvent. Initially, a mixture of bioactives and biopolymers is dissolved in a good solvent, and then this solution is 
injected into an antisolvent [107-109]. For hydrophobic biopolymers (such as zein or gliadin), the solvent is usually an organic liquid (such as ethanol, acetone, or supercritical carbon dioxide) whereas the antisolvent is water. Conversely, for hydrophilic biopolymers (such as whey protein), the solvent is usually water and the antisolvent is an organic liquid. When the solution surrounding the biopolymer molecules is changed from a good solvent to a poor solvent the biopolymer molecules associate with each other and form nanoparticles or microparticles. In this case, the bioactives are usually mixed with the biopolymer solution containing the good solvent, prior to injection into the poor solvent. As a result, they are trapped inside the biopolymer particles formed when the biopolymer molecules associate with each other in the poor solvent. A major advantage of the antisolvent approach is that relatively small bioactive-loaded microgels can be formed $(d<500 \mathrm{~nm})$, which is difficult using many other fabrication methods. The antisolvent precipitation method has been widely investigated for its potential to fabricate bioactive-loaded biopolymer particles from hydrophobic proteins, e.g., zein or gliadin [24, 110-112]. The bioactive molecules and hydrophobic proteins are dissolved within a concentrated ethanol solution, which is then titrated into an aqueous solution. This leads to the spontaneous generation of bioactive-loaded protein nanoparticles that can be used as is or converted into a powdered form.

The antisolvent precipitation method has been used to form microgels to encapsulate a wide range of bioactive agents, including carotenoids [113], curcumin [114, 115], quercetin [116]and resveratrol [117, 118]

\subsection{Particle Reduction Methods: Shearing and Grinding}

Microgels can be formed by breaking down macroscopic materials into smaller fragments using mechanical forces such as shearing or grinding [20, 39, 103, 119]. The dimensions and morphology of the microgels produced depends on the properties of the biopolymers, gelation mechanism, and mechanical forces used.

As discussed earlier, microgels can be formed from solutions of phase separated biopolymers by shearing them to create a W/W emulsion (particle formation), and then cross-linking them by changing environmental or solution conditions (particle gelation). The properties of the microgels formed depend on the intensity of the shearing forces used during the particle formation and gelation steps. As the shearing rate is increased, 
microgel size often decreases initially due to enhanced particle fragmentation, but then increases due to enhanced particle aggregation [87, 105]. Spherical microgels tend to be formed at relatively low applied shear rates, but ellipsoids or fibers are formed at relatively high shear rates due to shear-induced elongation of the particles $[119,120]$. The nature of the microgels produced using this approach also depends on other factors, such as solution viscosity, interfacial tension, and gelation rate relative to shear rate [103].

A microgel suspension could also be formed by creating a macroscopic gel consisting of bioactives trapped within a network of cross-linked biopolymer molecules, and then applying shearing forces to breakdown the system into small particles [15]. Alternatively, microgels could be fabricated using grinding methods that breakdown solid materials containing bioactives and biopolymers. For instance, an aqueous bioactive/biopolymer solution could be converted into a solid form using an appropriate drying method, and then converted into a powder containing small particles using a mechanical grinding device. Nevertheless, it is important to ensure that the biopolymer molecules are cross-linked at some stage during the production of the powder, otherwise the biopolymer molecules will simply dissolve when rehydrated.

\subsection{Selection of an appropriate fabrication method}

The various approaches that can be used to manufacture microgels have advantages and disadvantages that make them more or less suitable for particular applications. It is difficult to give specific guidelines about the selection of microgel fabrication methods, because each application has different requirements. For example, a particular application may require microgels with specific compositions, sizes, shapes, charges, and release characteristics. For this reason, only some general comments about the differences between different fabrication methods is given here. The range of sizes of microgels that can be produced is highly dependent on the fabrication method used: $d \approx$ 50 to $5000 \mu \mathrm{m}$ for simple injection methods; $d \approx 0.1$ to $100 \mu \mathrm{m}$ for inverse emulsion templating methods; $d \approx 0.1$ to $10 \mu \mathrm{m}$ for antisolvent precipitation methods; $d \approx 0.5$ to $100 \mu \mathrm{m}$ for electrostatic complexation; $d \approx 0.5$ to $100 \mu \mathrm{m}$ for thermodynamic incompatibility methods. The shape of microgels also depends on the fabrication method 
used, with some methods mainly producing spherical particles (such as emulsion templating), whereas others being capable of producing non-spherical particles (such as lithographic methods or phase separation methods with shearing). If microgels of a specific size and shape are required, then it will be necessary to select a fabrication method that is capable of producing these characteristics. The manufacturing method selected also determines the type of constituents needed to fabricate microgels. Injection methods require that the biopolymers can be cross-linked when injected into air or oil. Electrostatic complexation methods require the utilization of biopolymers that have opposite charges, such as negative (such as alginate, carrageenan, pectin, xanthan, and proteins above their isoelectric point) or positive (such as chitosan, polylysine, or proteins below their isoelectric point). Antisolvent precipitation methods require that a biopolymer be used that has good solubility in one type of food-grade solvent and poor solubility in another type (such as zein, which is soluble in ethanol solutions but insoluble in water). Thermodynamic incompatibility methods require the use of biopolymers that will spontaneously phase separate when mixed together under appropriate conditions in solution (such as maltodextrin and gelatin). If microgels of a specific composition are required, then it will be necessary to select a fabrication method that can be utilized with the desired components.

There are also considerable legal, economic, and practical differences in the utilization of different fabrication methods. The capital and running costs, the product throughput, scalability, environmental friendliness, and ease of implementation of different fabrication methods will determine their commercial viability. Consequently, many different factors have to be considered when selecting an appropriate fabrication method for a particular application.

\section{Microgel Particle Characteristics}

The retention, protection, and release of bioactive agents depend on the properties of the microgels that they are encapsulated within [17]. In this section, some of the major particle characteristics of microgels that can be altered to obtain specific functional attributes are highlighted. 


\subsection{Microgel Shape and Structure}

Most microgels tend to be spherical, but they can be fabricated to have different shapes if required, such as ellipsoids or fibers [103]. Non-spherical microgels can be prepared by applying mechanical forces (such as shearing) to the system during the particle formation and gelation processes, e.g., using the phase separation methods discussed in section 3.2.1 [120, 121]. Alternatively, non-spherical microgels can be fabricated using the lithographic methods discussed in section 3.2.2 [82]. The shape of a microgel has a major impact on a number of its functional attributes [122]. For example, fiber-like microgels tend to form highly viscous solutions at low effective volume fractions, but have rapid release characteristics. Conversely, spherical microgels tend to form lower viscosity solutions, but have slower release properties (at the same effective volume fraction). The reason for the faster release of an encapsulated component from a fiber-shaped microgel is that it has a smaller radius (but longer length) than a sphere of equivalent volume [123, 124].

The internal structure of microgels can also be controlled during the manufacturing process to alter their functional attributes. The interior of a microgel may be have the same composition throughout (homogeneous), or have some regions with one composition and other regions with a different composition (heterogeneous). The most common types of heterogeneous microgels have either core-shell or dispersion structures (Figure 7). The core-shell structure can be formed by co-extrusion of two gellingsolutions through an orifice [125]. Alternatively, coating homogeneous microgels with a layer of another material can form them, e.g., electrostatic deposition can be used to coat anionic microgels (such as calcium alginate beads) with a layer of cationic biopolymers (such as chitosan) [126, 127]. The dispersion structure can be formed by controlled phase separation of a biopolymer mixture, or by trapping small homogeneous microgel particles within larger microgel particles. For example, protein nanoparticles have been encapsulated in calcium alginate microgels to form composite microgel particles with a dispersion structure [128]. Typically, homogeneous beads are quicker and simpler to fabricate, but they have more limited functionality than heterogeneous beads. 


\subsection{Microgel Dimensions}

Microgels can be prepared with dimensions ranging from a few hundred nanometers to a few millimeters, depending on the constituents, fabrication method, and operating conditions used. In this section, the focus will be primarily on the impact of the external dimensions of the microgels (diameter) on their properties, but it should be noted that the dimensions of the internal structure of the microgels may also change [129], which will also influence their properties (Section 4.3). For many commercial applications it is important to carefully control the dimensions of the microgels, since particle size plays a major role in determining their functional attributes:

Appearance: The optical properties of a microgel suspension depend on the dimensions of the microgel particles compared to the wavelength of light (Section 5.1). Typically, light scattering is strongest when the particle dimensions are similar to the wavelength of light (around $500 \mathrm{~nm}$ ), and weaken for smaller or larger particles [130]. Consequently, microgels with dimensions around this value give suspensions that appear the most turbid or opaque. A suspension containing relatively small microgels $(d<100$ $\mu \mathrm{m})$ appears homogeneous to the human eye since they are below the limit of resolution, but larger microgels are discernable as discrete particles. It should be stressed that the optical properties of a microgel suspension also depend on the refractive index and concentration of the particles [130]. The degree of light scattering, and therefore opacity, tends to increase with increasing bead concentration and refractive index contrast.

Stability: The stability of a microgel suspension to gravitational separation and aggregation is strongly influence by particle dimensions (Section 5.2). In particular, the sedimentation rate of microgel particles increases as their dimensions increase, which may be an important consideration in applications where the viscosity of the medium surrounding the microgels is relatively low.

Mouthfeel: Human beings perceive ingested fluids as having either a smooth or grainy texture depending on the size, shape, and rheology of any particles present (Section 5.4). Consequently, it may be important to ensure that the microgels used in commercial products intended for oral ingestion are below a critical size (around $50 \mu \mathrm{m}$ ) to ensure that the product has a smooth and desirable mouthfeel. 
Retention and Release: Microgel dimensions have a major impact on their ability to retain and release bioactive agents (Section 5.5). Typically, the ability of a microgel to retain a bioactive agent increases as microgel dimensions increase. Conversely, the rate of bioactive release decreases with increasing microgel size, since the molecules then have a further distance to travel until they meet the surrounding medium. Careful control of microgel dimensions is therefore often needed to ensure an appropriate retention and release profile of an encapsulated bioactive agent.

Protection: The ability of microgels to protect encapsulated bioactives from chemical degradation also depends on their dimensions. The degradation of a bioactive agent is often initiated by specific components in the surrounding medium, e.g., the oxidation of encapsulated polyunsaturated lipids is promoted by transition metals in the surrounding water. In these cases, increasing the dimensions of the microgels leads to greater protection of the encapsulated bioactives because the contact area between the microgel surfaces and surrounding medium is reduced.

\subsection{Biopolymer Packing and Matrix Porosity}

The nature of the network of biopolymer molecules within the interior of microgel particles plays an important role in determining many of their functional attributes, including retention, release, protection, rheology, stability, and optical properties. The biopolymer chains may be linked together by covalent bonds or physical interactions, such as electrostatic, hydrogen bonding, or hydrophobic forces. The conformation and organization of the chains within the biopolymer network, as well as the number, strength, and location of the cross-links between them determines many of their properties, and should therefore be understood and controlled to create microgels with specific functional attributes.

The porosity of the biopolymer network inside microgels plays a major role in determining their ability to retain, protect, and release encapsulated components [131, 132]. The porosity ("mesh size") can be characterized in terms of the dimensions of the pores $(\zeta)$ between the biopolymer chains (Figure 8). The pore dimensions determine the rate at which bioactive molecules travel through the biopolymer network, and therefore they determine the retention and release of bioactive components (provided the bioactives do not strongly interact with the biopolymer molecules). If the bioactive dimensions are 
much larger than the pore dimensions, then they will be retained within the beads. Conversely, if the bioactive dimensions are much smaller than the pore dimensions, then they will easily diffuse through the biopolymer network and be released from the beads. The pore size of a hydrogel matrix can be related to the properties of the biopolymer molecules using appropriate models [133]. In addition, the release of bioactive agents from microgels can be predicted using appropriate mathematical models, which is useful for establishing the relative importance of specific biopolymer network properties on release rates (Section 5.5).

The packing on biopolymer molecules inside microgels can be described by a volume ratio, $R_{\mathrm{V}}[7]$.

$$
R_{V}=\frac{V_{\text {Microgel }}}{V_{\text {Biopolymers }}}
$$

Here, $V_{\text {Microgel }}$ is the volume occupied by the entire microgel particle (biopolymer chains + water) and $V_{\text {Biopolymers }}$ is the volume occupied by only the biopolymer chains within the microgel. The value of the volume ratio is close to unity for a microgel where the biopolymer molecules are densely packed so that there is little water present. On the other hand, the volume ratio may be much greater than unity for a system where the biopolymers are only loosely packed and there is a large amount of water present inside the beads. The packing of the biopolymer molecules within microgels influences the density, refractive index, and rheology of the beads, and thus impacts their stability to gravitational separation, their optical properties, and their mechanical properties (See Section 5). Studies indicate that the packing of biopolymers within microgels increases as the biopolymer and cross-linking concentration in the solutions used to fabricate them increases $[48,134]$. Just as with macroscopic gels, the packing of biopolymer chains within microgels depends on their concentration, the type and concentration of crosslinking agent used, and solution conditions (such as $\mathrm{pH}$, ionic strength, and temperature) [133]. Knowledge of the volume ratio of microgels is therefore often an important parameter to consider when designing delivery systems for bioactive agents. 


\subsection{Electrical Properties}

The electrical characteristics of microgels play an important role in determining their physicochemical properties, stability, and functional performance [7, 18]. The number and type of ionizable groups on the biopolymer molecules used to assemble microgel particles, as well as the nature of any associated ionic species (such as mineral ions), ultimately determine the electrical characteristics of microgels. In particular, the $\mathrm{pK}_{\mathrm{a}}$ values of the ionizable groups determines how the electrical properties of microgels changes with $\mathrm{pH}$. Microgel particles may have a negative, positive, or neutral charge depending on the type of biopolymers used to fabricate them. Anionic biopolymers, such as alginate, carrageenan, pectin and xanthan, have negatively charged groups (e.g., $\mathrm{COO}^{-}$or $-\mathrm{SO}_{4}^{-}$). Cationic biopolymers, such as chitosan or polylysine, have positively charged groups $\left(\right.$ e.g., $\left.-\mathrm{NH}_{3}{ }^{+}\right)$. The overall electrical characteristics of a microgel particle may be different from that of the biopolymers used to fabricate it due to charge neutralization and conformational effects. For example, the electrical properties of an alginate microgel will be different from those of the individual alginate molecules in solution because some of the anionic groups in the microgel are bound to calcium ions $[128,135]$. Moreover, if an anionic microgel (such as alginate) is coated with a cationic biopolymer (such as chitosan) the overall charge will be dominated by the cationic biopolymer chains that dangle into the aqueous phase [136].

The overall electrical properties of individual microgel particles is mainly determined by their surface potential, which depends on the nature of the charged molecules located at their surfaces [30]. The net charge on microgel particles governs their interactions with other microgel particles as well as other charged substances (such as biopolymers, mineral ions, lipid droplets, and surfaces). The surface potential of microgels can be conveniently characterized by measuring the change in $\zeta$-potential with $\mathrm{pH}$. The stability of microgel particles to aggregation depends on the strength of any repulsive or attractive electrostatic interactions operating between them (Section 5.2). To prevent microgel aggregation, it may be important to ensure that the $\zeta$-potential of the microgel particles exceeds a particular value to ensure that the electrostatic repulsion is stronger than any attractive interactions (such as van der Waals, hydrophobic, or depletion). The functional attributes of microgel particles can often be improved by 
coating them with a layer of charged biopolymers using an electrostatic deposition approach. In this case, it is important to ensure that the $\zeta$-potential of the microgels has the appropriate sign and is sufficiently high to ensure sufficient biopolymer attraction to the microgel surfaces.

The electrical characteristics of the interior of microgel particles is also important for many applications, and is mainly determined by the linear charge density of the biopolymer molecules in the gel network, as well as any associated mineral ions [29]. The internal electrical properties influence the retention and release of any charge bioactive agents. When the electrical potential of the bioactive agents is different from that of the biopolymer network (e.g., positive and negative), then they will be strongly attracted and therefore retained within the microgel. Conversely, when bioactive agents have the same sign of the electrical potential as the biopolymer network (e.g., negative and negative) they will be strongly repelled and therefore released from the microgel. This effect has been demonstrated for globular proteins encapsulated within alginate microgels [86], as discussed in the following section.

\subsection{Microenvironment}

The environment within a microgel may be very different from that of the surrounding medium, which can be utilized to control the retention, release and protection of encapsulated substances. In an aqueous solution, a bioactive agent is mainly surrounded by water molecules, but in a microgel it may be surrounded by a complex mixture of different molecular species, including biopolymers, mineral ions, buffering agents, antioxidants, and water molecules. Controlling the composition and properties of the interior of microgels can therefore be used to enhance the functional performance of bioactive components. For example, certain types of bioactives (e.g., enzymes and bacteria) tend to be more stable when their microenvironment is similar to that of their natural habitats. Enzymes are usually found in cells or tissues that have a specific $\mathrm{pH}$, ionic strength, and osmotic pressure. Consequently, it may be beneficial to design the interior of microgels so that their microenvironment is optimized to enhance the stability of any encapsulated components [137].

Many bioactive agents are chemically stable over a certain range of $\mathrm{pH}$ values but unstable under another. For example, many enzymes are denatured and lose activity 
when exposed to low or high $\mathrm{pH}$ values [8]; $\beta$-carotene degrades under highly acidic conditions [138]; curcumin degrades under neutral and alkaline conditions [139]; probiotics are deactivated under highly acid conditions [4]. The stability of these bioactive agents could be improved by encapsulating them in microgels that have an internal $\mathrm{pH}$ that is different from that of the external $\mathrm{pH}$.

Computer simulations show that the $\mathrm{pH}$ and ion distribution within the interior of a hydrogel matrix may be quite different from that of the surrounding aqueous phase, which alters the ionization of both the biopolymer molecules and bioactive agents [29, 140]. For example, the $\mathrm{pH}$ inside a hydrogel matrix consisting of anionic biopolymers may be appreciably less than that of the surrounding aqueous phase. This effect arises because of the strong electrostatic repulsion between the negatively charged groups on the biopolymer chains in the hydrogel network. The system reduces the magnitude of this repulsion by increasing the local proton concentration, thereby causing some of the anionic groups to become protonated and lose their charge. The magnitude of the difference in $\mathrm{pH}$ between the inside and outside of the biopolymer matrix increases with decreasing ionic strength and increasing biopolymer charge density [29]. This phenomenon may be utilized to retain charged bioactive molecules (such as proteins or peptides) inside microgels over a certain range of external $\mathrm{pH}$ conditions, but then release them under another set of $\mathrm{pH}$ conditions. Alternatively, it could be used to protect encapsulated bioactive components from $\mathrm{pH}$-induced chemical degradation by ensuring that the internal $\mathrm{pH}$ is maintained at a value where the bioactive is stable. Nevertheless, the ionic strengths in many commercial products (such as foods) are relatively high, and therefore the $\mathrm{pH}$ gradient may not be sufficient for many applications.

Buffering agents can also be utilized to control the internal $\mathrm{pH}$ of microgels. Recent experimental studies in our laboratory have shown that the incorporation of an insoluble basic buffer (magnesium hydroxide) inside calcium alginate beads creates a $\mathrm{pH}$ inside the beads that can be appreciably different from that of the $\mathrm{pH}$ of the external solution [141]. Magnesium hydroxide $\left(\mathrm{Mg}(\mathrm{OH})_{2}\right)$ is insoluble at neutral $\mathrm{pH}$, but dissolves under acidic conditions. Consequently, when $\mathrm{Mg}(\mathrm{OH})_{2}$-loaded microgel particles are exposed to a highly acidic gastric environment the microenvironment within the beads remains close to neutral because as hydrogen ions $\left(\mathrm{H}^{+}\right)$enter the beads they are neutralized by hydroxyl 
ions released from the buffer $\left(\mathrm{OH}^{-}\right)$. Studies have shown that the $\mathrm{pH}$ inside the microgel particles can be maintained neutral for more than 2 hours under simulated gastric conditions. This approach therefore has considerable potential for protecting bioactive components that are unstable under acidic gastric conditions, such as some enzymes and probiotics. Indeed, this approach has recently been shown to be capable of stabilizing lipase and lactase from deactivation in simulated gastric fluids, and retaining their activity in simulated intestinal fluids.

The nature of the microgel microenvironment may also impact the retention and release of certain bioactive components. If the bioactive components bind strongly to the biopolymer network, then they will tend to be retained within the microgel particles. Conversely, if there is a strong repulsion between the bioactive molecules and the biopolymer network, then the bioactive molecules will tend to be released (provided they can diffuse through the pores in the hydrogel matrix). For food-grade microgels, the forces acting between the bioactive components and the biopolymer network are usually non-specific physical interactions, such as electrostatic, hydrophobic, or hydrogen bonding. Electrostatic interactions are one of the most common types of physical interaction used to control the retention and release of bioactive components in microgel particles. For example, the electrical charge on protein molecules changes from positive to negative as the $\mathrm{pH}$ is increased from below to above their isoelectric point (pI). Consequently, proteins will tend to be retained within anionic microgels at relatively low $\mathrm{pH}$ values (where the beads are negative and the proteins are positive), but be released at relatively high $\mathrm{pH}$ values (where both the beads and proteins are negative). Recent studies have shown that proteins or protein-coated lipid droplets are retained within anionic alginate beads under acidic conditions, but released under alkaline conditions due to this effect $[135,142]$.

In principle, various other types of functional ingredients can be incorporated into microgels to improve the stability of encapsulated bioactive agents or to control release/retention, such as antioxidants, chelating agents, prebiotics, lipid droplets, solid particles, or non-gelling biopolymers. For example, antioxidant proteins (caseins) have been co-encapsulated with bioactive lipids (w-3 oils) inside alginate beads to improve their chemical stability [97]. Lipid droplets or solid particles could be incorporated 
within microgels to increase the diffusion path length (tortuosity), and thereby slow down the release of encapsulated bioactives. To the authors knowledge, there have been few studies on the rational design of microgel interiors to create specific microenvironments for protection, retention, or release of bioactives, but this should be a fruitful area of future research.

\section{Physicochemical Properties of Microgel Suspensions}

In this section, a brief overview of the physicochemical properties of microgel suspensions is given because this has important implications for many of their commercial applications. There have been relatively few systematic studies in this area, and this would certainly be a fruitful area for future work.

\subsection{Optical Properties}

The optical properties of a microgel suspension often determine the type of products it can be successfully utilized in. For example, commercial food products may be transparent (e.g., some soft drinks), slightly turbid (e.g., some sauces), or opaque (e.g., some dressings). In general, a particle suspension may have an appearance that varies from transparent to opaque depending on the size, concentration, and refractive index of the particles it contains [130]. Moreover, the human eye can discern the presence of discrete particles when their dimensions exceed about $100 \mu \mathrm{m}$ (the limit of resolution). Consequently, it may be important to carefully control the properties of the particles in a microgel suspension to obtain a desirable appearance in the final product.

The most important factors influencing the overall appearance of a microgel suspension are the concentration, size, and refractive index of the microgels, as well as the type and concentration of any chromophores present [130]. The microgels affect appearance due to their ability to scatter light waves in different directions, whereas the chromophores affect appearance because of their ability to preferentially absorb light waves at specific wavelengths. For dilute systems, the appearance is often simply characterized in terms of the turbidity, which depends on the fraction of light transmitted through the system. For concentrated systems, the appearance can be conveniently quantified in terms of tristimulus color coordinates, such as the $L^{*} a^{*} b^{*}$ system [143]. 
The $L^{*}$ value represents lightness, which varies from 0 (black) to 100 (white). The $a^{*}$ and $b^{*}$ values are color coordinates, with $+a^{*}$ corresponding to red, $-a^{*}$ corresponding to green, $+b^{*}$ corresponding to yellow, and $-b^{*}$ corresponding to blue. Thus the color of a suspension can be defined by specifying the three color coordinates. Mathematical models have been developed to relate the optical properties of a colloidal suspension to the characteristics of the particles and chromophores it contains [130]. Expressions for suspension turbidity $(\tau)$ and tristimulus color coordinates $\left(\mathrm{L}^{*}, \mathrm{a}^{*}\right.$, and $\mathrm{b}^{*}$ ) can be simply represented by the following expressions:

$$
\begin{aligned}
& \tau=\mathrm{f}(\phi, d, \Delta n, \alpha(\lambda), \lambda) \\
& L^{*}, a^{*}, b^{*}=\mathrm{f}(\phi, d, \Delta n, \alpha(\lambda))
\end{aligned}
$$

Here $\phi$ is the particle concentration, $d$ is the particle diameter, $\Delta n$ is the refractive index difference between the particles and surrounding medium, $\alpha(\lambda)$ is the visible absorption spectrum of the chromophores, $\lambda$ is the wavelength of light, and $f()$ means "a function of'. A more detailed description of the mathematical models that can be used to relate the optical properties of suspensions to particle properties is given elsewhere [130, 144]. Mathematical models (such as the Mie theory) are also available on-line that can be used to analyze the optical properties of colloidal suspensions (www.philiplaven.com/mieplot.htm). These models require information about the structure, composition, and properties of the system, such as the size, structure, shape, refractive index, and concentration of the particles, as well as the absorption spectra of the chromophores.

The dependence of the lightness of suspensions on particle concentration, size, and refractive index are shown schematically in Figure 9. Typically, the lightness increases as the particle concentration increases, has a maximum value at a particular particle size (around the wavelength of light), and increases as the refractive index contrast increases. Experimental measurements of the optical properties of microgel suspensions support these predictions. For example, the lightness of biopolymrt microgel suspensions has been reported to increase with increasing particle concentration and refractive index 
contrast $[145,146]$. Typically, the lightness increases steeply from around 0 to $5 \%$ particles and then increases more gradually or levels out at higher particle concentrations (Figure 9). Studies have shown that the lightness of suspensions of swollen starch granules is much lower than that of protein particles, which was attributed to the fact that the starch granules contained much more water, and therefore had a lower refractive index contrast [147, 148]. The lightness of microgel suspensions fabricated from caseinate and pectin was shown to increase when the packing of the biopolymers in the microgel particles increased due to an increase in ionic strength [146].

\subsection{Stability}

Microgel suspensions are thermodynamically unstable systems that will eventually breakdown through a number of physicochemical mechanisms, with the most important being gravitational separation and aggregation [7].

\subsubsection{Gravitational separation}

Microgel particles typically have a different density than the surrounding medium and may therefore move downwards (sedimentation) or upwards (creaming) due to gravitational forces. The most common form of gravitational separation in microgel suspensions is sedimentation because the beads usually have a higher density than the surrounding aqueous phase, but creaming may occur when the beads contain a high level of low-density particles (such as oil droplets or air bubbles). The densities of microgel particles depends on the density and concentration of the different components inside them $[20,106]$.

In general, the sedimentation velocity of an isolated rigid spherical particle in an ideal liquid is given by Stokes' law:

$$
v_{\text {Stokes }}=\frac{g d^{2}\left(\rho_{2}-\rho_{1}\right)}{18 \eta_{1}}
$$

Here, $v_{\text {Stokes }}$ is the sedimentation velocity, $g$ is the gravitational acceleration constant, $d$ is the particle diameter, $\rho_{2}$ is the density of the particles, $\rho_{1}$ is the density of the surrounding aqueous phase, and $\eta_{1}$ is the shear viscosity of the surrounding aqueous phase. The sign of $v$ specifies the direction that the particle tends to move due to gravity: downwards (+) or upwards (-). This expression indicates that the sedimentation velocity increases with 
increasing particle size, increasing density contrast, and decreasing continuous phase viscosity. Most types of microgel particles tend to be relatively large (> $10 \mu \mathrm{m})$, and therefore they will tend to sediment rapidly when dispersed within pure water (Table 2). Indeed, experimental studies have shown that different types of microgel particles tend to sediment rapidly when dispersed in aqueous solutions due to their relatively high density and large size, e.g., chitosan [149], pectin/caseinate [106], alginate [86], and carrageenan [56]. Consequently, they may only be suitable for application in highly viscous or gelled products. If microgel particles are going to be utilized in low viscosity products, then it is important to ensure that have small dimensions and low biopolymer levels in order to inhibit sedimentation. Alternatively, it may be possible to retard sedimentation by density matching the beads to the surrounding water (see later) [106]. Stokes' Law is only strictly applicable for dilute suspensions of non-interacting rigid spheres dispersed in an ideal fluid. More sophisticated theories are required to take into account the effects of particle size distributions, non-spherical particles, particle fluidity, particle interactions, and non-Newtonian fluids [150].

Many microgel suspensions are relatively concentrated systems, and therefore particle interactions may impact their sedimentation stability. The following semiempirical expression can be used to predict the sedimentation velocity of particles in concentrated suspensions:

$$
v=v_{\text {Stokes }}\left(1-\frac{\phi_{e f f}}{\phi_{C}}\right)^{k \varphi_{c}}
$$

Typically, $\phi_{\mathrm{c}}$ is taken to be the volume fraction $(\approx 0.585)$ at which spherical particles become close packed and $k$ is as an adjustable parameter with a value around 5.4 [151]. This equation predicts that the sedimentation velocity of the microgel particles decreases as the effective volume fraction increases, until the particles become so close packed and then they cannot move any further because of jamming effects. Thus a concentrated microgel suspension may be stable to gravitational separation because the particles are so closely packed together. Indeed, experimental studies have shown that concentrated microgel suspensions form viscoelastic solids [152], which would therefore be expected to be stable to sedimentation. 
Microgel particles typically contain a number of constituents that contribute to the overall particle density, including biopolymers, water, and oil droplets. The density of a multi-component microgel particle can be estimated using the following expressing assuming that ideal mixing occurs:

$$
\rho_{\text {particle }}=\sum_{i} \phi_{i} \rho_{i}
$$

Here, $\phi_{\mathrm{i}}$ and $\rho_{\mathrm{i}}$ are the volume fraction and density of the $i^{\text {th }}$ component. Thus, for a microgel particle that contains biopolymers $(\mathrm{B})$, water $(\mathrm{W})$ and oil droplets $(\mathrm{O})$ the overall particle density is:

$$
\rho_{\text {bead }}=\phi_{B} \rho_{B}+\phi_{W} \rho_{W}+\phi_{o} \rho_{O}
$$

Here, $\phi_{\mathrm{B}}+\phi_{\mathrm{W}}+\phi_{\mathrm{O}}=1$. The above equation can be rearranged to give the density contrast between the bead and the surrounding water:

$$
\Delta \rho_{\text {microgel }}=\phi_{B} \Delta \rho_{B}+\phi_{O} \Delta \rho_{O}
$$

Here, $\Delta \rho_{\text {microgel }}\left(=\rho_{\text {microgel }}-\rho_{\mathrm{W}}\right), \Delta \rho_{\mathrm{B}}\left(=\rho_{\mathrm{B}}-\rho_{\mathrm{W}}\right)$ and $\Delta \rho_{\mathrm{O}}\left(=\rho_{\mathrm{B}}-\rho_{\mathrm{W}}\right)$ are the density contrasts of the microgel particles, biopolymers, and oil with water. This equation shows that the density contrast of a microgel particle depends on its composition and the density contrast of the biopolymers and oils present. Typically, the density of biopolymer chains is around $1600 \mathrm{~kg} \mathrm{~m}^{-3}$, the density of oil is around $920 \mathrm{~kg} \mathrm{~m}^{-3}$, and the density of water is around $1000 \mathrm{~kg} \mathrm{~m}^{-3}$, thus $\Delta \rho_{\mathrm{B}}=600 \mathrm{~kg} \mathrm{~m}^{-3}$ and $\Delta \rho_{\mathrm{O}}=-80 \mathrm{~kg} \mathrm{~m}^{-3}$ (but these values depend on oil type, biopolymer type, and aqueous phase composition). In the absence of oil droplets, microgel particles will always tend to sediment because they are denser than water, with the rate of sedimentation increasing with biopolymer concentration (Table 1). However, sedimentation can be prevented if the density of the beads is made to be the same as the surrounding aqueous phase $\left(\Delta \rho_{\text {microgel }}=0\right)$ by incorporating a sufficiently 
high level of oil droplets. Density matching can be achieved when the oil to biopolymer ratio in the microgel particles has the following value:

$$
\frac{\phi_{O}}{\phi_{B}}=-\frac{\Delta \rho_{B}}{\Delta \rho_{O}}
$$

This would suggest that the oil level inside the microgel particles should be about $7.5 \times$ higher than the biopolymer level. This approach has been used to improve the stability of biopolymer microgel particles to gravitational separation [106]. Sedimentation of caseinpectin microgel particles was inhibited by increasing the amount of oil droplets encapsulated inside them to reduce the density contrast (Figure 10). Density matching of microgel particles may be essential for those commercial applications where the viscosity of the final product should remain relatively low.

\subsubsection{Particle aggregation}

\subsubsection{Colloidal interactions}

Another factor that is particularly important in determining the stability and properties of microgel suspensions is the tendency for the beads to aggregate with each other or with other kinds of particles. In general, the balance of attractive and repulsive forces that act between the particles determines the aggregation stability of colloidal suspensions [7]. The most important types of colloidal interactions for microgel particles are summarized below:

$$
w(h)=w_{V D W}(h)+w_{E}(h)+w_{S}(h)+w_{H}(h)+w_{D}(h)
$$

Here $w(h)$ refers to the interaction potential at a surface-to-surface particle separation of $h$, and the subscripts VDW, E, S, H and D refer to van der Waals, electrostatic, steric, hydrophobic, and depletion interactions, respectively.

Van der Waals: This type of force acts between all types of colloidal particles and is usually attractive, long range, and relatively strong. To a first approximation the van der Waals interaction potential ( $\mathrm{W}_{\mathrm{VDW}}$ ) is given by the following expression: 


$$
w_{V D W}(h)=-\frac{A r}{12 h}
$$

Here, $r$ is the radius of the microgel particles and $A$ is the Hamaker function. The Hamaker function depends on the physicochemical properties of the particles and the surrounding medium [150]. Typically, the magnitude of the Hamaker function increases as the difference in the refractive index and dielectric constant of the particles and surrounding medium increase. Microgel particles typically contain a considerable amount of water and so the van der Waals interactions may be relatively weak because the refractive index and dielectric constant contrast is relatively low. As an example, the Hamaker functions for microgel particles with biopolymer contents of $10 \%, 25 \%, 50 \%$, $75 \%$ and $100 \%$ were calculated to be $0.034,0.21,0.84,1.92$ and $3.59 \mathrm{kT}$ using the equations given elsewhere [150]. The impact of microgel composition on the strength and range of van der Waals attraction is shown in Figure 11a. As the biopolymer level in the microgel particles decreases, the van der Waals attraction decreases.

Electrostatic: This type of interaction plays an important role in determining the stability of microgel particles to aggregation. To a first approximation, the electrostatic interaction potential $\left(\mathrm{w}_{\mathrm{E}}\right)$ between two similarly charged spheres is given by the following expression (assuming constant surface charge):

$$
w_{E}(h)=-2 \pi \varepsilon_{R} \varepsilon_{0} r \psi^{2} \ln (1-\exp (-\kappa h))
$$

Here, $\varepsilon_{0}$ is the dielectric constant of a vacuum, $\varepsilon_{\mathrm{R}}$ is the relative dielectric constant of the aqueous phase, $\psi$ is the surface electrical potential of the particles, and $\kappa$ is the reciprocal of the Debye screening length, which determines the distance that the electrical field radiates into the surrounding aqueous solution. For aqueous solutions around room temperature, $\kappa^{-1} \approx 0.304 / \sqrt{ } I \mathrm{~nm}$, where $I$ is the ionic strength expressed in moles per liter [30]. The electrostatic interaction is repulsive when the particles involved have similar signed charges (both negative or both positive), but attractive when they have opposite charges. The range of the electrostatic interaction is strongly dependent on ionic 
strength, e.g., $\kappa^{-1}=0.30,3$ and $30 \mathrm{~nm}$ for ionic strengths of 1000, 10, and $0.1 \mathrm{mM}$, respectively. The impact of ionic strength on the electrostatic repulsion between two microgel particles $(r=10 \mu \mathrm{m})$ is shown in Figure 11b. The strength and range of the electrostatic repulsion decreases as the ionic strength increases, which is important to know because the salt level in many commercial products varies considerably. For many types of microgel particles, the electrostatic interaction is also highly dependent on $\mathrm{pH}$ since many of the charged groups on the biopolymer molecules have functional groups with dissociation constants ( $\mathrm{pK}_{\mathrm{A}}$ values) in the $\mathrm{pH}$ range typically found in commercial products ( $\mathrm{pH} 2$ to 8 ). The impact of $\mathrm{pH}$ on the electrical characteristics of the beads is usually characterized by measuring the change in $\zeta$-potential with $\mathrm{pH}$. The importance of electrostatic interactions is clearly shown for protein-based microgels, such as whey protein or zein particles dispersed in water, which may be fairly stable to aggregation at $\mathrm{pH}$ values far from their isoelectric point ( $\mathrm{pI}$ ) due to strong electrostatic repulsion, but unstable at the $\mathrm{pI}$ due to weak electrostatic repulsion $[153,154]$. Theoretical models based on thermodynamic analysis has been developed to describe the electrostatic interactions between microgel particles in the presence of monovalent ions [155].

Steric: Steric interactions are usually relatively strong short-range repulsive interactions that only become important when the microgel particles come into close proximity. To a first approximation the steric interaction potential $\left(w_{\mathrm{S}}\right)$ can be given by the following expressions:

$$
\begin{array}{ll}
w_{S}(h)=k T\left(\frac{2 \partial}{h}\right)^{12} & \text { for } \mathrm{h}<\delta \\
w_{\mathrm{S}}(h)=0 & \text { for } \mathrm{h} \geq \delta
\end{array}
$$

Here, $\delta$ is taken to be the thickness of the outer layer of biopolymers at the surfaces of the microgel particles, which will be approximately equal to the radius of hydration of the biopolymers in solution. The steric repulsion is negligible when the microgel particles are not in contact, but increases steeply as soon as they come close enough for the outer layer of biopolymer molecules to overlap (Figure 11c). The range of the steric repulsion increases as the dimensions of the biopolymer molecules at the outer surfaces increases. In practice, the magnitude of the steric repulsion is likely to depend on the rheological properties of the beads, being stronger for more rigid beads [14]. In addition, it depends 
on the solvent quality of the biopolymers - if the solvent is poor then the outer layer of biopolymers may actually be attracted to each other.

Hydrophobic: Hydrophobic interactions are only important in microgel particles that have appreciably amounts of non-polar groups exposed on their surfaces. The contact between these non-polar groups and water is thermodynamically unfavorable due to the hydrophobic effect, and so the system tries to reduce the contact area by bringing the non-polar groups together. In systems with an appreciable surface hydrophobicity, this interaction manifests itself as a relatively strong and long-range attractive force. The strength of the attraction typically increases as the surface hydrophobicity of the microgel particles increases. This type of interaction is important for hydrophobic proteins (such as gliadin and zein), which are often used to form microgel particles by the antisolvent precipitation method $[24,156]$. After formation, the protein particles have a strong tendency to aggregate with each other due to the strong hydrophobic attraction associated with their high surface hydrophobicity. It is for this reason, that this type of microgel particle is often stabilized by adding emulsifiers to the aqueous phase so as to adsorb to the bead surfaces and cover any non-polar patches $[110,156]$.

Depletion: Depletion interactions are important in systems where there is a relatively high concentration of non-adsorbed colloids in the aqueous phase surrounding the microgel particles. These colloids are usually highly hydrated biopolymer molecules (such as polysaccharides), but they may also be surfactant micelles or protein aggregates. In this section, we only consider non-adsorbed biopolymers because they are likely to be the most common type of colloids found in microgel suspensions. The non-adsorbed biopolymers are excluded from a narrow zone (approximately equal to the radius of hydration) surrounding each of the microgel particles. Consequently, there is a region around each of the microgel particles where the non-adsorbed biopolymer level is close to zero, and another region in the aqueous phase when it is relatively high. This concentration gradient leads to an osmotic pressure that tends to drive the microgel particles together. The strength of the depletion attraction typically increases as the concentration of non-adsorbed biopolymer molecules increases, as well as their relative 
volume increases. Depletion flocculation of the microgel particles will occur when the depletion attraction overcomes any repulsive interactions.

Overall interaction: The overall aggregation stability of the microgel particles is therefore a balance of the various attractive and repulsive forces that operate in the particular system, which will depend on the composition of both the microgel particles and the surrounding aqueous phase, as well as on the particle dimensions. Theoretical predictions of the van der Waals, electrostatic, steric and total interactions between two microgel particles are shown in Figure $12(\mathrm{I}=10 \mathrm{mM}, r=10 \mu \mathrm{m}, \delta=20 \mathrm{~nm})$. These predictions show that the curves for the steric and total interactions are very similar, which suggests that steric interactions dominate the overall interaction for microgels.

\subsubsection{Flocculation and Coalescence}

Microgel particles may become aggregated when the attractive forces acting between them dominate the repulsive forces [7]. The particles flocculate when they stick together, but each particle retains its original integrity, but they coalesce when two or more particles merge together to form a larger particle. Whether flocculation or coalescence occurs depends on the nature of the colloidal interactions operating in the system, as well as on the molecular and physicochemical properties of the biopolymer network that makes up the surface and interior of the microgel particles. Both flocculation and coalescence can be prevented by manipulating the colloidal interactions so that the repulsive interactions outweigh the attractive interactions. This can be achieved in a number of ways depending on the system:

- Increase the electrostatic repulsion: There are a number of ways to increase the electrostatic repulsion between microgel particles. The repulsion can be enhanced by increasing the magnitude of the surface potential ( $\zeta$-potential) on the particles, e.g., by using biopolymers with a high charge density or by altering the solution $\mathrm{pH}$. Alternatively, it can be achieved by decreasing the ionic strength of the aqueous solution surrounding the microgel particles, and especially by avoiding the presence of multivalent counter-ions. 
- Increase the steric repulsion: This can be achieved by ensuring that the microgel particles are coated by a layer of biopolymer molecules that extend a relatively large distance into the surrounding aqueous phase or that they have a high elastic modulus.

- Reduce the hydrophobic attraction: The hydrophobic attraction can be reduced by decreasing the surface hydrophobicity of the microgel particles. This can be achieved by using highly hydrophilic biopolymers to assemble the particles, or by covering any exposed non-polar regions using surfactants or biopolymers.

- Reduce the depletion attraction: The magnitude of the depletion attraction can be reduced by decreasing the concentration of any non-adsorbed biopolymers in the aqueous phase surrounding the microgel particles.

- Reducing any bridging mechanisms: Microgel particles may become aggregated when a single biopolymer adsorbs to the surfaces of two or more particles. For example, a single positively charged biopolymer may adsorb to the surfaces of a number of different negatively charged microgel particles causing them to become linked together. This process can be inhibited by ensuring that there are no oppositely charged biopolymers present in the aqueous phase surrounding the beads.

Bead coalescence can be inhibited by ensuring that there are strong cross-links between the biopolymer molecules within the interior of the microgel particles, or by covering the microgel particles with a protective coating $[87,106]$. Bead coalescence is a major problem in the formation of microgel particles by both thermodynamic incompatibility and complex coacervation methods (Section 3.3). In these methods, the biopolymer-rich particles generated during the particle-formation stage have to be rapidly stabilized against coalescence by quickly gelling or coating them $[103,157,158]$. It should be noted that bead coalescence is not always undesirable, since it may be utilized to create novel structures or textures within microgel suspensions. For instance, the biopolymer-rich particles can be allowed to grow to a particular desired size before they are cross-linked, or non-spherical microgel particles can be produced by shearing the biopolymer-rich particles before cross-linking [103]. 
Mathematical models have been developed to calculate the colloidal interactions between particles, and thus predict the stability of particle suspensions to aggregation [30, 159]. These models can be used to predict the influence of particular particle characteristics (such as dimensions, surface potential, surface hydrophobicity, and interfacial thickness) and solution conditions (such as $\mathrm{pH}$, ionic strength, and nonabsorbing biopolymers) on aggregation stability. The development of stable microgel suspensions often depends on identifying the dominant attractive and repulsion interactions operating in a particular system, and then developing effective strategies to control them.

\subsection{Rheology}

The rheological properties of microgel particles and their suspensions are important for a number of reasons. First, the rheology of the microgel particles themselves influences their functional attributes, such as stability and release properties. Second, microgel particles are incorporated into commercial products (such as foods, supplements, or pharmaceuticals) that are expected to have certain textural characteristics, such as a low viscosity liquid, a high viscosity cream, a semi-solid gel, or an elastic solid. Consequently, the final product should have the desired textural characteristics after the microgel particles are incorporated. Third, the processing of microgel suspensions often depends on their rheology as this influences their mixing characteristics and their ability to flow through pipes and into containers.

\subsubsection{Rheology of Individual Microgel Particles}

Microgel particles are typically semi-solid materials with both solid-like and liquidlike characteristics. The rheology of an individual microgel particle can be characterized by the relationship between the applied stress (force per unit area) and the resulting strain (normalized deformation) $[160,161]$. The initial slope of a plot of stress versus strain provides information about the elastic modulus, which is related to particle softness or hardness. Microgel particles may also be characterized by their breaking stress or strain. The breaking stress is related to the minimum force that can be applied to a particle before it fractures, whereas the breaking strain is related to the amount a particle can be deformed before it fractures. Microgel particles that fracture at small forces are fragile, 
whereas those that fracture at large forces are sturdy. Microgel particles that fracture at small strains are brittle, whereas those that fracture at large strains are rubbery. Knowledge of the rheology of individual microgel particles is important for certain applications because it determines their stability during storage and transport, as well as their tendency to breakdown when subjected to mechanical forces in the gastrointestinal tract (such as in the mouth, stomach, or small intestine). The rheology of microgel particles can be manipulated by controlling the type and concentration of biopolymer and cross-linking agents used $[160,162]$. Analytical approaches for measuring the rheology of individual microgel particles have been reviewed, and include methods such as deformation in shear flow, optical tweezers, micromanipulation, and atomic force microscopy $[161,163]$.

\subsubsection{Rheology of Microgel Suspensions}

When microgel particles are incorporated into a liquid (such as water) they typically increase the viscosity because they perturb the fluid flow profile leading to more energy dissipation due to friction [152]. The amount that they increase the viscosity by depends primarily on the effective volume fraction and interactions of the microgel particles [164166].

To a first approximation the viscosity of a dilute suspension of non-interacting microgel particles can be described by the modified Einstein equation:

$$
\eta=\eta_{1}\left(1+2.5 \phi_{\mathrm{eff}}\right)
$$

where

$$
\phi_{e f f}=\phi R_{V}=\frac{C R_{V}}{\rho_{B}}
$$

Here, $\eta_{1}$ is the viscosity of the pure solvent (usually water), $\phi$ is the volume fraction occupied by the biopolymer chains within the entire system, $R_{\mathrm{V}}$ is the volume ratio (volume of microgel particles/volume of biopolymer chains), $c$ is the biopolymer concentration within the entire system, and $\rho_{\mathrm{B}}$ is the density of the biopolymer chains (around $1600 \mathrm{~kg} \mathrm{~m}^{-3}$ ). The Einstein equation was originally derived to describe the shear viscosity of dilute suspensions of rigid spherical particles, but it provides a reasonable 
prediction of the viscosity of dilute microgel suspensions $\left(\phi_{\text {eff }}<5 \%\right)$. The above equations predict that the viscosity of a microgel suspension increases as the biopolymer concentration increases, but also as the volume ratio of the microgel particles increases. Thus microgels that are able to trap large amounts of solvent inside are much more effective at increasing the viscosity. The volume ratio is closely related to the swelling ratio $(Q)$ used in some viscosity models i.e., the mass of hydrated biopolymer over the mass of dry biopolymer [167]. Knowledge of the density of the microgel and water are needed to convert these two parameters: $R_{\mathrm{V}}=Q \rho_{\mathrm{M}} / \rho_{\mathrm{B}}$. Theoretical models based on statistical thermodynamics have been developed to relate polymer characteristics to the swelling ratio of both neutral and charged systems [18, 168, 169]

In many practical applications, the effective volume fraction of microgel particles is considerably higher than $5 \%$ and therefore the Einstein equation is no longer applicable because it does not take into account particle-particle interactions. In concentrated systems, the following semi-empirical equation has been developed to relate the viscosity of a microgel suspension to particle properties [167]:

$$
\eta=\eta_{1}\left(1-\frac{\phi_{e f f}}{\phi_{C}}\right)^{-2}
$$

In this equation, $\phi_{\mathrm{C}}$ is taken to be a critical packing parameter $(\approx 0.64)$, which is the concentration where the particles become randomly jammed closely together and the system gains some solid-like characteristics [166]. The value of $\phi_{C}$ can be calculated from the measured particle size distribution of a particle suspension using a suitable model $[167,170,171]$. Theoretical predictions have shown that the value of $\phi_{C}$ may vary from around 0.64 for a monodisperse system to 0.97 for a highly polydisperse system [170]. The above equation was originally developed for hard spheres, but experimental studies with microgel suspensions have shown that it is also applicable to soft spheres up to $\phi_{\mathrm{C}}[167]$. In other words, the deformability (elastic modulus) of the individual microgel particles does not appear to affect the rheological properties until the particles become tightly packed together.

Predictions made using the above equation indicate that the relative viscosity of microgel suspensions increases moderately when $\phi_{C}$ increases from about 0 to $40 \%$, but 
then increases steeply as the effective volume fraction of the microgel particles increases towards the critical packing parameter (Figure 13a). The biopolymer concentration where the viscosity first increases steeply decreases as the volume ratio (or swelling ratio) of the microgel particles increases (Figure 13b). This has important implications for some commercial applications since it means that relatively low levels of biopolymer could lead to a large increase in viscosity or even to gel-like characteristics if the microgel particles have very open structures [152]. Above $\phi_{\mathrm{C}}$, a microgel suspension exhibits viscoelastic and plastic behavior, with the values of the elastic modulus and yield stress increasing with increasing particle concentration, as shown in Figure 14 for nonfood grade microgel suspensions [172]. In this regime, the rheological properties of the microgel suspension also depend on the rheological properties of the individual microgel particles, with harder spheres giving higher elastic moduli and yield stresses [152].

The above equations assume that there are no strong attractive or repulsive interactions between the microgel particles [150]. Typically, there is an increase in suspension viscosity in the presence strong attractive interactions because the microgel particles become associated with each other (which increases their effective volume fraction). There is also an increase in suspension viscosity in the presence of strong repulsive interactions because this increases the effective volume occupied by the beads (which decreases the critical packing parameter).

\subsection{Mouthfeel}

The incorporation of microgel particles into commercial products intended for oral ingestion may influence their perceived mouthfeel $[39,173]$. When the particles have diameters below a critical size (around $50 \mu \mathrm{m}$ ) they cannot be detected as discrete particles and the product has a smooth or creamy mouthfeel, but when they are larger they are detected as discrete particles and the product has a rough or grainy mouthfeel. The critical size where particles are detected as discrete entities depends on their rheology and shape, as well as on the viscosity of the surrounding liquid [173-175]. Hard particles are more easily detected as individual entities within the mouth than soft particles $[173,174]$. Non-spherical particles are perceived more easily than spherical ones $[173,176]$. The presence of discrete particles in a suspension becomes easier to 
discern as the viscosity of the surrounding liquid decreases [173, 175]. The size, shape, and rheology of microgel particles may therefore be an important consideration when applying them in certain commercial products intended for oral ingestion.

\subsection{Retention and Release Properties}

Typically, a bioactive agent should be retained within the microgel particles under one set of environmental conditions, but then released when it is exposed to another set of environmental conditions (such as $\mathrm{pH}$, ionic strength, temperature, or enzyme activity). In addition, it is often important to control the release profile of the bioactive agents from the microgel particles.

Bioactive agents may be held within microgel particles through a number of mechanisms: (i) physical entrapment inside small pores; (ii) attractive physical interactions between the bioactive and the biopolymer network; (iii) covalent bonding of the bioactive to the biopolymer network. The bioactive agents can then be released from microgel particles through numerous physicochemical mechanisms, including diffusion, dissolution, weakening of molecular interactions, erosion, fragmentation, and swelling [133, 177] (Figure 15). The selection of specific retention and release mechanisms depends on the particular application. The physicochemical basis and mathematical description of different release mechanisms is described in detail elsewhere [7, 123, 124, 178], and so only a brief summary of each mechanism is given here.

\subsubsection{Diffusion}

A bioactive agent may be released from a microgel particle by simple molecular diffusion through the pores in the biopolymer network [179]. In the simplest case, the bioactive molecules are initially fully dissolved and evenly distributed throughout the particles, they are not strongly attracted or repelled by the biopolymer network, and the structure of the biopolymer network does not change throughout the diffusion process. Under these circumstances, the main factors influencing the rate of release of a bioactive agent from microgel particles due to diffusion are: bioactive properties (such as molecular dimensions); biopolymer network properties (such as pore size); solvent properties (such as viscosity); particle characteristics (such as size, shape, and structure); bioactive concentration gradient between the interior and exterior of the beads; and, fluid 
flow conditions. Typically, the rate of bioactive release increases as the molecular dimensions of the bioactive decreases, the pore size of the biopolymer network increases, and the microgel particle diameter decreases. In practice, the diffusion of bioactive molecules out of microgel particles may be more complicated than this simple situation. The bioactive agent may initially be present in an insoluble form that is unevenly dispersed throughout the microgel particles. The bioactive agents may interact strongly with the biopolymer network through attractive or repulsive interactions, such as electrostatic, hydrophobic, or hydrogen bonding (Figure 15b). The biopolymer network may change its structure throughout the release process, e.g., due to swelling, shrinking, erosion, or fragmentation (Figure 15a). These factors influence the release rate of the bioactive molecules, and should be taken into account when developing suitable mathematical models to predict release profiles. In practice, simple diffusion is unlikely to be a suitable release mechanism for most applications of microgel beads because the size of the bioactive molecules is much smaller than the pore size of the biopolymer network, and so the bioactive molecules are rapidly released.

The release of bioactive components from porous microgel particles can be estimated using the following equation [180]:

$$
\Phi=\frac{M(t)}{M(\infty)}=1-\frac{6}{\pi^{2}} \sum_{n=1}^{\infty} \frac{1}{n^{2}} \exp \left(-\frac{D_{g e l} n^{2} \pi^{2} t}{a^{2}}\right)
$$

Here, $\Phi$ is the fraction of the bioactive agents that have diffused out of the microgel beads by time $t, M(t)$ and $M(\infty)$ are the bioactive concentrations in the microgel particles at time $t$ and at equilibrium (infinite time), $n$ is an integer, $a$ is the radius of the microgel particles, and $D_{\text {gel }}$ is the diffusion coefficient of the bioactives through the biopolymer network within the particles (see later).

The hindered diffusion of bioactive agents through the polymer networks can be described by the following equation $[169,181]$ :

$$
D_{g e l}=D_{w} \exp \left(-\pi\left(\frac{r_{H}+r_{f}}{\xi+2 r_{f}}\right)\right)
$$


Here, $D_{\mathrm{w}}$ is the translational diffusion coefficient of the bioactive agents through pure water, $r_{\mathrm{H}}$ is the hydrodynamic radius of the bioactive agents, $r_{\mathrm{f}}$ is the cross-sectional radius of the biopolymer chains in the hydrogel network, and $\zeta$ is the diameter of the pores in the hydrogel network. The relevant structural parameters of calcium alginate particles have been reported in the literature: pore diameter $(\zeta)=4$ to $400 \mathrm{~nm}$; alginate chain radius $\left(r_{\mathrm{f}}\right)=0.83 \mathrm{~nm}$ [169]. To a first approximation, the translational diffusion coefficient of the bioactive components through pure water can be estimated using the expression:

$$
D_{\mathrm{w}}=k_{\mathrm{B}} T / 6 \pi \eta r_{\mathrm{H}}
$$

Here, $k_{\mathrm{B}}$ is Boltzmann's constant, $T$ is absolute temperature, and $\eta$ is the viscosity of the water. The above equations can be utilized to predict the influence of microgel particle properties (such as external dimensions and pore size) and bioactive characteristics (such as size) on the rate of bioactive release. Predictions of the influence of microgel particle dimensions on the release of bioactive agents are shown in Figure 16, assuming that the bioactive agents and pores had similar sizes $(r=50 \mathrm{~nm})$. As expected, the release rate decreased as the size of the microgel particles increased because the bioactive molecules had a longer distance to travel through the biopolymer network before being released. Microgel particles with different dimensions may therefore be used modulate the release profile of encapsulated bioactives.

The impact of pore size on the release of a bioactive agent $\left(r_{\mathrm{H}}=50 \mathrm{~nm}\right)$ from microgel particles $(r=100 \mu \mathrm{m})$ is shown in Figure 17a. These predictions show that as the pore size of the beads increases, the release rate of the encapsulated bioactives increases. The release rate is relatively slow when the bioactive agents have dimensions appreciably smaller than the pore size, but is relatively faster otherwise. Consequently, one would not expect microgel particles to be particularly effective at retaining relatively small bioactive agents. A more direct indication of the impact of microgel pore diameter on the release of bioactive molecules is shown in Figure 17b, which shows plots of $D_{\text {gel }} / D_{\mathrm{w}}$ versus pore radius. These calculations provide an indication of the ability of the biopolymer network to retard the movement of the bioactive molecules through the 
microgel particles. The predictions indicate that the bioactive agents must have dimensions appreciably larger than the pore diameter before their diffusion is appreciably retarded. Thus, densely packed microgel particles with very small pore sizes would be required to retain relatively small bioactive molecules through this mechanism. Conversely, bioactive components dissolved within relatively large oil droplets $(r=1000$ $\mathrm{nm}$ ) should be completely retained within microgel beads with typical pore sizes. An example of the ability to control the release of bioactive agents from microgel beads based on altering the pore size is shown in Figure 17c, where curcumin-loaded lipid droplets were encapsulated in alginate microgels prepared using different alginate concentrations [54]. A higher alginate concentration leads to small pore sizes, and slower droplet release.

The use of mathematical models to predict the release of bioactive components from microgel particles is particularly useful for designing delivery systems, as it enables the impact of specific factors, such as particle size, pore size, and bioactive size to be investigated in a systematic fashion [123, 124, 182, 183].

\subsubsection{Dissolution}

In some situations, the rate-limiting step determining the release of a bioactive agent from a microgel particle is the dissolution of one of its major constituents, which could be the bioactive agent itself or the microgel particle that contains it [183]. Many bioactive agents and microgel particles are insoluble under one set of conditions, but soluble under another set. Thus, a crystalline or amorphous bioactive agent can be dispersed into a microgel particle in the form of solid particles, where it is retained due to its relatively large size compared to the pores in the biopolymer network. However, when the environmental conditions are altered the solid particles dissolve, thereby releasing the bioactive molecules so that they can diffuse through the biopolymer network and be released from the particles. When the dissolution rate is much slower than the diffusion rate, then dissolution will control the overall release rate. This approach has recently been used in our laboratory to encapsulate a buffering agent (magnesium hydroxide) inside alginate microgels: the $\mathrm{Mg}(\mathrm{OH})_{2}$ is insoluble under neutral conditions, but dissolves and is released under acidic conditions. 
Alternatively, changes in the solubility of the microgel particles can be used to control the release of the encapsulated bioactive agents. The microgel particles may be fabricated from biopolymers that are insoluble under one set of conditions, but dissolve under another set. Consequently, the encapsulated bioactive agents are released once the biopolymer network dissolves [179]. In this case, the overall release rate of the bioactive is governed by the dissolution rate of the microgel particles, which depends on the nature of the internal biopolymer network (e.g., number and strength of cross-links), particle properties (e.g., size, shape and structure), and environmental conditions (e.g., dilution factor, solvent type, $\mathrm{pH}$, ionic strength, and temperature). Typically, dissolution occurs more rapidly when the microgel particle dimensions decrease because this increases the surface area exposed to the surrounding liquids. This approach is widely used in powdered forms of bioactive-loaded microgel particles formed by spray drying: the bioactive is trapped within a biopolymer network in a solid particle, but is released when the particles are added to water and the biopolymers dissolve [184].

\subsubsection{Molecular interactions}

A bioactive agent may be released from microgel particles due to an alteration in the strength of the interactions between the bioactive agents and the biopolymer network [86, 185]. In this case, the interior of the microgel particles is designed so that there is initially a relatively strong attraction between the bioactive agents and the biopolymer network, which prevents them from diffusing out of the particles (Figure 15b). However, when the microgel particles encounter specific environmental conditions (such as a particular $\mathrm{pH}$ range, salt level, or temperature), the attractive interactions are weakened, so the bioactive agent can then diffuse out of the microgel particles (provided it is sufficiently small compared to the pore size). Different kinds of molecular interaction can be used for this purpose, including electrostatic, hydrophobic, and hydrogen bonding, but electrostatic interactions are the most widely utilized. In this case, the bioactive agents are retained inside the microgel beads when they have an opposite charge to the biopolymer network, e.g., a positive bioactive and a negative biopolymer network. The bioactive agents are then released by altering the $\mathrm{pH}$ so that their charge becomes similar to that of the biopolymer network, e.g., a negative bioactive and negative biopolymer 
network. Alternatively, the bioactive agents can be released by increasing the ionic strength so as to weaken the attractive electrostatic interactions through electrostatic screening effects. It should be noted that the electrical characteristics of both the bioactive and biopolymer network may be different within the interior of a microgel particle than they are when simply dispersed in water, which has to be taken into account when designing a suitable release mechanism [29].

An example of this phenomenon is shown in Figure 18 for proteins (whey proteins) encapsulated within anionic microgel (alginate) particles [86]. At $\mathrm{pH}$ values below their isoelectric point, the proteins are positively charged whereas the biopolymer network is negatively charged, which leads to protein retention due to a strong electrostatic attraction. When the $\mathrm{pH}$ is adjusted so that it is above the isoelectric point, both the proteins and biopolymer network are negatively charged, which causes the proteins to be released. Similar results have been reported for protein-coated lipid droplets encapsulated in anionic microgels, but the pores in the biopolymer network have to be larger to release lipid droplets [142]. Consequently, this approach could be used to trigger the release of both hydrophilic and hydrophobic bioactives by appropriate design of the microgel particles.

\subsubsection{Erosion}

In this case, the bioactive agent is released from the microgel particles due to erosion of the biopolymer network when it encounters specific environmental conditions [179]. Typically, erosion occurs due to chemical or enzymatic cleavage of the molecules within the biopolymer network, which may occur primarily at the surfaces of the particles (surface erosion) or throughout the interior of the particles (bulk erosion). Initially, the bioactive agent is distributed throughout the microgel particles, but is released when the biopolymer network erodes (Figure 15a). In most commercial applications, the erosion of microgel particles occurs due to either chemical (e.g., strong acids or bases) or enzymatic (e.g., lipases, proteases, or amylases) hydrolysis of the biopolymer chains. The release profile of the bioactive agent is then governed by the rate at which surface or bulk erosion occurs, which depends on microgel properties (e.g., size, composition and structure) and environmental conditions (e.g., $\mathrm{pH}$ and enzyme activity). The overall release profile depends on the relative magnitude of the erosion and diffusion rates: when 
erosion occurs slower than diffusion the overall release is erosion-limited, but when diffusion occurs slower than erosion the overall release is diffusion-limited.

In microgel particles intended for oral ingestion, one of the most common mechanisms utilized for bioactive release is enzyme digestion of the biopolymer network. Microgel particles fabricated from proteins may be digested by proteases in the stomach or small intestine [186-189], whereas those fabricated from starches may be digested by amylases in the mouth, stomach, or small intestine [190, 191]. Conversely, erosion of microgels in the upper GIT can be inhibited by utilizing dietary fibers to fabricate them, since these are resistance the enzymes in the mouth, stomach, and small intestine [23, 192]. Knowledge of the enzyme hydrolysis of different biopolymers can therefore be utilized to fabricate microgel delivery systems with different retention and release characteristics for GIT applications (Figure 19) [35].

\subsubsection{Fragmentation}

In this case, the bioactive agent is released from the microgel particles when they become fragmented due to applied physical, chemical, or enzymatic processes (Figure 15a). The release rate of the bioactive agent then depends on the fracture properties of the particles, such as the breaking stress, and size and shape of the fragments formed. After particle fragmentation has occurred the bioactives may released by diffusion, dissolution or erosion processes, but the release rate will be faster because of the greater surface area and decreased particle dimensions. Fragmentation is an important factor to consider when creating microgel particles designed to release bioactive agents in response to specific mechanical forces, such as the mastication process that occurs in the mouth [80].

\subsubsection{Swelling}

In this case, the release of the bioactive agent is triggered by an increase in the pore size of the biopolymer network when the microgel particles absorb solvent from the surroundings and swell $[185,193,194]$. The bioactive agent is initially trapped within microgel particles that have pore sizes small enough to prevent their movement. However, the particles are designed to swell when they encounter a particular set of environmental conditions (such as $\mathrm{pH}$, ionic strength, or temperature), which causes an 
increase in pore size and release of the bioactive agent. The overall release rate depends on the relative magnitudes of the swelling rate and diffusion rates. One of the most common methods of promoting the swelling of microgel particles is based on the utilization of changes in electrostatic interactions. When the electrostatic interactions between biopolymer chains are attractive they can come into fairly close proximity, and so the pore size in the biopolymer network is relatively small. However, when the electrostatic interactions are repulsive the biopolymer chains attempt to get as far away from each other as possible, and so the pore size becomes relatively large, leading to bead swelling. Changes in the degree of swelling of microgels due to electrostatic interactions can therefore be used to create $\mathrm{pH}$ - or salt-induced triggered release of bioactive components [168, 195, 196].

\subsubsection{Designing release profiles}

The design of a microgel particle for a particular application relies on establishing the most appropriate release profile for the bioactive agent involved, e.g., burst, delayed, sustained, or triggered (Figure 20). Once this has been established then the composition and structure of the microgel particles can be designed to give this release profile based on one of the release mechanisms described earlier. For instance, a bioactive agent could be delivered to the colon by designing a microgel particle that remained intact and impermeable within the upper GIT (mouth, stomach, and small intestine), but that was eroded by enzymes released by colonic bacteria in the large intestine (Figure 19). Alternatively, an acid-labile bioactive agent could be delivered to the small intestine by creating a microgel particle that remained intact and impermeable in the mouth and stomach, but that swelled, dissociated or eroded in the small intestine due to a change in $\mathrm{pH}$ or enzyme activity

The release profile of a bioactive agent can be expressed as the change in its concentration at the required site of action as a function of time. Numerous parameters can be derived from the release profile, including the total area under the curve (AUC), the maximum bioactive concentration released $\left(C_{\max }\right)$, and the incubation time required to reach this maximum bioactive concentration $\left(t_{\max }\right)$ [197]. The composition and structure of microgel particles must be carefully controlled to obtain the desired release profile. The release profile may be achieved using a single type of microgel particle, or by using 
mixtures of microgel particles with different characteristics (e.g., sizes and compositions). Alternatively, microgel particles may be mixed with other types of delivery systems (such as nanoemulsions) to create specific release profiles [50].

Mathematical models are available to predict the release profile of bioactive components from microgel particles due to various release mechanisms, e.g., diffusion, dissolution, and erosion [198-203]. Information about the appropriate physicochemical attributes of the system is typically required to utilize these models, e.g., particle size distributions, particle shapes, pore sizes, diffusion coefficients, partition coefficients, concentration gradients, and fluid flow conditions. This information can sometimes be found in the published scientific literature, or it can often be measured for the specific system that is being utilized. The rational design of microgel particles for a particular release application typically depends on establishing the nature of the dominant release mechanism, then quantifying the major factors that impact the release rate, and using appropriate mathematical theories to describe these processes.

\subsection{Protection}

It is often assumed that when a bioactive component is encapsulated within a microgel particle it will be protected from its surrounding environment, but this is typically not the case. Microgel particles are highly porous and therefore it is relatively easy for small molecules (such as oxygen, enzymes, iron ions, hydrogen ions, or hydroxyl ions) to diffuse in and out of them (Section 5.5.1). Consequently, bioactive molecules trapped within the interior of a microgel particle may still be prone to chemical or enzyme degradation if small molecules in the surrounding solution can diffuse into them. For instance, acid-labile enzymes encapsulated within microgel particle are still prone to deactivation when they are exposed to gastric fluids, since the hydrogen ions can still rapidly diffuse through the biopolymer network. It may therefore be necessary to design the interior of the microgel particles to create a microenvironment that can protect any encapsulated substances. Some potential strategies are highlighted below:

- Antioxidants: Antioxidant components can be packed into the interior of microgel particles to inhibit the degradation of bioactive agents that are susceptible to oxidation, such as polyunsaturated fatty acids or carotenoids. 
- Buffers: Buffering agents can be incorporated into the microgel interior to create an internal $\mathrm{pH}$ that is different to the external $\mathrm{pH}$, thereby protecting bioactive agents that are susceptible to $\mathrm{pH}$-induced degradation.

- Chelating agents: Chelating agents (such as EDTA, organic acids, or some biopolymers) could be incorporated into a microgel particles to bind transition metals (such as iron or copper) than normally promote oxidation reactions.

- Colloids: Colloids (such as lipid, protein, polysaccharide, or mineral particles) could be trapped inside a microgel particle so as to change the diffusion path length of any detrimental substances that might diffuse into them.

\section{Applications of Microgel Particles}

In this section, a brief overview of some of the applications of microgel particles for the encapsulation, protection, and release of bioactive components is given. There have been a huge number of publications in this area recently, and so only a few representative examples are given to highlight the potential of microgel systems. Special emphasis is given to microgel particles that are formed from food-grade biopolymers (proteins and/or polysaccharides) because these are suitable for encapsulation of a variety of bioactive agents intended for oral ingestion.

\subsection{Pharmaceuticals}

Microgel particles have been investigated for their ability to encapsulate, protect, and release various types of pharmaceutical agents, including small molecules, peptides, proteins, DNA, stem cells, and bacteria [58, 204-206]. Alginate-based microgels are commonly used for these applications due to their ease of preparation and mild fabrication conditions [207]. This type of microgel particle has been used to encapsulate a wide range of pharmaceutical agents, including bioactive proteins and peptides [16, 207], bioactive lipids [208], and small molecule drugs [209, 210]. Other types of biopolymers have also been used to fabricate microgel particles suitable for utilization in the pharmaceutical industry, such as chitosan [211], cellulose [212, 213], pectin [214217], starch [218, 219], and carrageenan [27, 220]. Chitosan-based microgels have been fabricated for the encapsulation and controlled delivery of ibuprofen [221], $\alpha$-lipoic acid 
[222], insulin [223] and protein antigens [224]. Microgel particles fabricated from cellulose and its derivatives have been used to encapsulate a wide range of pharmaceutical agents [225]. Live cells (fibroblasts) have been encapsulated in gelatinbased microgels produced using microfluidics [226]. Enzyme- and pH-responsive microgel particles have been developed for the oral delivery of siRNA to target inflammation in the GIT [227]. A chemotherapy drug (5-Fluorouracil) has been encapsulated in pectin-coated chitosan-microgel particles, and was shown to be released under simulated GIT conditions [227]. It was claimed that these microgel particles may be useful for the treatment of colorectal tumors. Microgel particles formed from alginate and chitosan have been used to encapsulate an anti-ulcerative colitis drug (5aminosalicylic acid) so that it could pass through the upper GIT and be delivered to the colon [228]. As mentioned earlier, this is just a few examples of many thousands of studies that have been published in the literature.

\subsection{Nutraceuticals}

Microgel particles have also been widely investigated for their potential to encapsulate, protect, and release nutraceuticals. Calcium alginate microgels have been used to encapsulate $\beta$-carotene-enriched lipid droplets [229]. Encapsulation of the carotenoids into the microgels improved their stability to chemical degradation when stored at elevated temperatures and within the GIT. However, microgel encapsulation reduced the bioaccessibility of the carotenoids by inhibiting lipid digestion, which decreased the amount of carotenoids solubilized in the mixed micelle phase. Nevertheless, the carotenoids encapsulated in the microgel particles still had a relatively high bioaccessibility, and so these systems would be useful as delivery systems for this type of highly hydrophobic nutraceutical. Calcium alginate and potassium carrageen microgels have been used to encapsulate curcumin-enriched lipid droplets [49]. The carrageenan microgels had relatively fragile structures that were easily deformed and disrupted under simulated GIT conditions, thereby releasing the curcumin-loaded lipid droplets. Conversely, alginate microgels had a more robust structure that retained the lipid droplets throughout the GIT. The bioaccessibility of the curcumin was therefore considerably higher when encapsulated in carrageenan microgels than in the alginate ones. Consequently, different types of beads could be used to obtain different retention 
and release characteristics. The impact of alginate type, inulin addition, and bead dimensions on the encapsulation and release of bioactive polyphenols has recently been examined [230]. This study showed that microgel composition and structure could be optimized to obtain the desired retention and release properties. Polyphenols from a variety of plant sources have been encapsulated in calcium alginate/chitosan microgels [52]. The polyphenols were shown to maintain their antioxidant activity after encapsulation, which is important for their practical applications as nutraceuticals.

The ability of food-grade microgels to retain and release encapsulated proteins in response to a $\mathrm{pH}$ trigger was recently demonstrated [135]. A model globular protein (whey protein) was trapped within alginate microgels using $\mathrm{pH}$ conditions where there was an electrostatic attraction between the cationic protein and anionic alginate molecules $(\mathrm{pH}<\mathrm{pI})$. The proteins were then released when the $\mathrm{pH}$ was increased above the isoelectric point because of electrostatic repulsion between the anionic protein and anionic alginate molecules under these conditions (Figure 18). Calcium alginate microgels have also been shown to control the retention and release of protein-coated lipid droplets in response to $\mathrm{pH}$ changes by a similar mechanism [142]. This approach enables the $\mathrm{pH}$-triggered release of hydrophobic bioactive molecules from microgels.

Lipid droplets have been encapsulated within microgel particles so as to control lipid digestion in the small intestine $[48,83]$. Encapsulation in alginate microgels greatly decreased the rate and extent of lipid digestion under simulated small intestine conditions, which may be important for applications where lipophilic bioactives need to be delivered to the colon. The rate of lipid digestion decreased as the pore size of the biopolymer network inside the microgels decreased, which was attributed to inhibition of lipase diffusion. In addition, the lipid digestion rate decreased with increasing microgel particle diameter, which was attributed to the greater path length that the lipase molecules had to diffuse to reach the lipid droplets in the interior of the particles. Consequently, the lipid digestion profile can be tuned by using microgel particles with different characteristics. Alternatively, the lipid digestion rate can be controlled using mixed delivery systems, e.g., lipid-loaded microgels and lipid nanoparticles [50]. 


\subsection{Probiotics}

Probiotics are bacteria that are beneficial to human health when ingested orally [4, $231,232]$. It is postulated that these bacteria reach the large intestine and alter the balance of bacterial species in the colon. Consequently, there is interest in the oral delivery of specific probiotics to the human colon using supplements or functional foods. Indeed, there are many commercial supplement and food products that already contain probiotics. One of the major challenges to the incorporation of probiotics into these products is that they are living organisms that may lose their viability during storage or during passage through the GIT. In particular, many types of probiotics are highly susceptible to degradation when exposed to acids, enzymes, or bile salts in the stomach and small intestine. Effective delivery systems are therefore needed to encapsulate probiotics and protect them from degradation in foods and in the upper GIT, but then release them within the colon [233-235].

A number of studies have investigated the potential of food-grade microgels for the encapsulation, protection and release of probiotics. Pectin microgels have been shown to increase the viability of a probiotic (Lactobacillus rhamnosus) in both in vitro and in vivo studies [236]. Encapsulation may have created a beneficial microenvironment for the bacteria, or prevented components in the gastrointestinal fluids from interacting with them. Alginate microgels have recently been shown to improve the viability of another probiotic species (Bifidobacteria longum) [237, 238]. Cellulose microgels were recently shown to be more effective than alginate microgels at improving the retention and viability of a probiotic (Lactobacillus plantarum) [239].

\subsection{Enzymes}

Microgels can also be used to retain or control the activity of encapsulated enzymes. Encapsulation of lactase ( $\beta$-galactosidase) into $\kappa$-carrageenan-based microgels has been shown to increase its stability to changes in $\mathrm{pH}$ and temperature [56]. This effect was attributed to the difference in the microenvironment of the enzyme inside and outside the microgels, particularly the fact that there was a relatively high potassium concentration inside the biopolymer network, and this ion is known to be essential for proper lactase functioning. Lactase has also been encapsulated in biopolymer microgels consisting of a 
mixture of carboxymethyl cellulose (CMC) and alginate [240]. Incorporating CMC into the microgels was shown to increase the loading, stability, and activity of the enzyme. Studies have shown that lipase encapsulated within alginate microgels retains its activity, which is important for developing strategies that allow the repeated utilization of enzymes in industrial applications [241]. Recently, our laboratory has been interested in utilizing biopolymer microgels to protect enzymes from deactivation as they pass through the GIT. In particular, many enzymes are denatured and deactivated when exposed to the highly acidic gastric juices in the stomach. Conventional microgels are unsuitable for this purpose because small hydrogen ions $\left(\mathrm{H}^{+}\right)$easily diffuse through the biopolymer network and reduce the internal $\mathrm{pH}$ of the microgels. It was shown that the interior of microgels could be maintained close to neutral conditions by trapping a basic buffer (magnesium hydroxide) within them (Figure 21). This buffer is insoluble under neutral or alkaline conditions, but dissolves under acid conditions. Consequently, when hydrogen ions from the gastric fluids enter the microgels, the local $\mathrm{pH}$ is temporarily decreased, which causes some of the $\mathrm{MgOH}_{2}$ to dissolve, thereby releasing $\mathrm{OH}^{-}$ions that interact with the $\mathrm{H}^{+}$ions and so keeping the internal $\mathrm{pH}$ close to neutral. It was shown that these microgels could be used to retain the activity of both lipase and lactase under simulated gastric conditions.

\subsection{Flavors}

Microgel particles can be used to control the release profile of encapsulated flavors (taste and aroma molecules) to control the flavor profile of foods and other products. Alginate microgels have been utilized to encapsulate liquid smoke flavor [53]. The liquid smoke is retained inside the particles at low temperatures, but is released when they are heated above a certain temperature. Alginate microgels have also been used to encapsulate flavor oils, such as thyme oil, so as to improve their dispersibility and stability in foods [52]. Microgel particles of different sizes and compositions have been shown to be able to control the release of taste and aroma molecules under simulated oral conditions [80, 242]. The effectiveness of the microgels at controlling flavor release depended on the characteristics of the flavor molecules, such as their molecular weight and polarity. 


\section{Conclusions}

Microgel particles have considerable potential for the encapsulation, protection, and controlled delivery of bioactive agents, such as pharmaceuticals, supplements, and nutraceuticals. The size, shape, structure, composition, and electrical properties of microgel particles can be tailored so as to obtain specific functional attributes. Hydrophilic bioactives are typically directly mixed with the biopolymer solution used to form the microgel particles, whereas hydrophobic bioactives need to be trapped in hydrophobic carriers (such as micelles, oil droplets, or vesicles) first. The retention and release of the encapsulated bioactives depends on the pore size of the biopolymer network inside the microgel particles, as well as any attractive or repulsive interactions between the bioactives and biopolymers. Microgel particles can be fabricated entirely from food-grade ingredients using simple processing operations, and may therefore be suitable for large scale commercial application. The development of microgels as encapsulation systems for bioactive agents is a rapidly growing area and considerable advances are being made in their design, fabrication, characterization, and utilization for this purpose. For food and supplement applications, it will be important to identify systems that can be fabricated entirely from food-grade biopolymers using economically viable fabrication methods, and to ensure that these microgel particles maintain their functionality within actual commercial products and the human GIT.

\section{Acknowledgements}

This material was partly based upon work supported by the Cooperative State Research, Extension, Education Service, USDA, Massachusetts Agricultural Experiment Station (MAS00491) and USDA, NRI Grants (2013-03795).

\section{References}

[1] Ensign LM, Cone R, Hanes J. Oral drug delivery with polymeric nanoparticles: The gastrointestinal mucus barriers. Advanced Drug Delivery Reviews. 2012;64:557-70. 
[2] Mitragotri S, Burke PA, Langer R. Overcoming the challenges in administering biopharmaceuticals: formulation and delivery strategies. Nature Reviews Drug Discovery. 2014;13:655-72.

[3] Thanki K, Gangwal RP, Sangamwar AT, Jain S. Oral delivery of anticancer drugs:

Challenges and opportunities. Journal of Controlled Release. 2013;170:15-40.

[4] Cook MT, Tzortzis G, Charalampopoulos D, Khutoryanskiy VV. Microencapsulation of probiotics for gastrointestinal delivery. Journal of Controlled Release. 2012;162:56-67.

[5] Renukuntla J, Vadlapudi AD, Patel A, Boddu SHS, Mitra AK. Approaches for enhancing oral bioavailability of peptides and proteins. International Journal of Pharmaceutics. 2013;447:75-93.

[6] McClements DJ. Advances in fabrication of emulsions with enhanced functionality using structural design principles. Current Opinion in Colloid \& Interface Science. 2012;17:235-45.

[7] McClements DJ. Nanoparticle- and Microparticle-based Delivery Systems. First ed. Boca Raton, FL: CRC Press; 2015.

[8] Whitehurst RJaVO, M. Enzymes in Food Technology. Chichester, West Sussex, UK: John Wiley and Sons; 2010.

[9] Berton-Carabin CC, Ropers MH, Genot C. Lipid Oxidation in Oil-in-Water Emulsions: Involvement of the Interfacial Layer. Comprehensive Reviews in Food Science and Food Safety. 2014;13:945-77.

[10] Arab-Tehrany E, Jacquot M, Gaiani C, Imran M, Desobry S, Linder M. Beneficial effects and oxidative stability of omega-3 long-chain polyunsaturated fatty acids. Trends in Food Science \& Technology. 2012;25:24-33.

[11] Al-Sheraji SH, Ismail A, Manap MY, Mustafa S, Yusof RM, Hassan FA. Prebiotics as functional foods: A review. Journal of Functional Foods. 2013;5:1542-53.

[12] Corona-Hernandez RI, Alvarez-Parrilla E, Lizardi-Mendoza J, Islas-Rubio AR, de la Rosa LA, Wall-Medrano A. Structural Stability and Viability of Microencapsulated Probiotic Bacteria: A Review. Comprehensive Reviews in Food Science and Food Safety. 2013;12:614-28.

[13] Lima AC, Sher P, Mano JF. Production methodologies of polymeric and hydrogel particles for drug delivery applications. Expert Opinion on Drug Delivery. 2012;9:23148.

[14] Dickinson E. Microgels - An alternative colloidal ingredient for stabilization of food emulsions. Trends in Food Science \& Technology. 2015;43:178-88.

[15] Farjami T, Madadlou A. Fabrication methods of biopolymeric microgels and microgel-based hydrogels. Food Hydrocolloids. 2017;62:262-72.

[16] Liu AL, Garcia AJ. Methods for Generating Hydrogel Particles for Protein Delivery. Annals of Biomedical Engineering. 2016;44:1946-58.

[17] Torres O, Murray B, Sarkar A. Emulsion microgel particles: Novel encapsulation strategy for lipophilic molecules. Trends in Food Science \& Technology. 2016;55:98108.

[18] Saunders BR, Laajam N, Daly E, Teow S, Hu XH, Stepto R. Microgels: From responsive polymer colloids to biomaterials. Advances in Colloid and Interface Science. 2009;147-48:251-62.

[19] McClements DJ. Recent progress in hydrogel delivery systems for improving nutraceutical bioavailability. 2017;Food Hydrocolloids. 
[20] Matalanis A, Jones OG, McClements DJ. Structured biopolymer-based delivery systems for encapsulation, protection, and release of lipophilic compounds. Food Hydrocolloids. 2011;25:1865-80.

[21] Stephen AJ, Phillips GO, Williams PA. Food Polysaccharides and Their Applications. Second Edition ed. Boca Raton, FL.: CRC Press; 2006.

[22] Coviello T, Matricardi P, Marianecci C, Alhaique F. Polysaccharide hydrogels for modified release formulations. Journal of controlled release. 2007;119:5-24.

[23] Singh B, Bala R. Polysaccharide based hydrogels as controlled drug delivery system for GIT cancer. International Journal of Biological Macromolecules. 2014;65:524-33.

[24] Patel AR, Velikov KP. Zein as a source of functional colloidal nano- and microstructures. Current Opinion in Colloid \& Interface Science. 2014;19:450-8. [25] Zhong QX, Jin MF. Zein nanoparticles produced by liquid-liquid dispersion. Food Hydrocolloids. 2009;23:2380-7.

[26] George M, Abraham TE. Polyionic hydrocolloids for the intestinal delivery of protein drugs: alginate and chitosan - a review. Journal of Controlled Release. 2006;114:1-14.

[27] Li L, Ni R, Shao Y, Mao SR. Carrageenan and its applications in drug delivery. Carbohydrate Polymers. 2014;103:1-11.

[28] Turgeon SL, Schmitt C, Sanchez C. Protein-polysaccharide complexes and coacervates. Current Opinion in Colloid \& Interface Science. 2007;12:166-78.

[29] Longo GS, Szleifer I. Adsorption and protonation of peptides and proteins in $\mathrm{pH}$ responsive gels. Journal of Physics D-Applied Physics. 2016;49.

[30] Israelachvili J. Intermolecular and Surface Forces, Third Edition. Third Edition ed. London, UK: Academic Press; 2011.

[31] Srivastava R, Brown JQ, Zhu H, McShane MJ. Stable encapsulation of active enzyme by application of multilayer nanofilm coatings to alginate microspheres. Macromolecular bioscience. 2005;5:717-27.

[32] Norton JE, Espinosa YG, Watson RL, Spyropoulos F, Norton IT. Functional food microstructures for macronutrient release and delivery. Food \& Function. 2015;6:663-78. [33] Sensoy I. A Review on the Relationship Between Food Structure, Processing, and Bioavailability. Crit Rev Food Sci Nutr. 2014;54:902-9.

[34] Grundy MML, Edwards CH, Mackie AR, Gidley MJ, Butterworth PJ, Ellis PR. Reevaluation of the mechanisms of dietary fibre and implications for macronutrient bioaccessibility, digestion and postprandial metabolism. British Journal of Nutrition. 2016;116:816-33.

[35] Zhang Z, Zhang R, Chen L, Tong Q, McClements DJ. Designing hydrogel particles for controlled or targeted release of lipophilic bioactive agents in the gastrointestinal tract. European Polymer Journal. 2015;72:698-716.

[36] Joye IJ, McClements DJ. Biopolymer-based nanoparticles and microparticles: Fabrication, characterization, and application. Current Opinion in Colloid \& Interface Science. 2014.

[37] Shewan HM, Stokes JR. Review of techniques to manufacture micro-hydrogel particles for the food industry and their applications. Journal of Food Engineering. 2013;119:781-92. 
[38] Zhang ZP, Zhang RJ, Chen L, Tong QY, McClements DJ. Designing hydrogel particles for controlled or targeted release of lipophilic bioactive agents in the gastrointestinal tract. European Polymer Journal. 2015;72:698-716.

[39] Burey P, Bhandari BR, Howes T, Gidley MJ. Hydrocolloid gel particles: Formation, characterization, and application. Crit Rev Food Sci Nutr. 2008;48:361-77.

[40] Oxley JD. Spray cooling and spray chilling for food ingredient and nutraceutical encapsulation. In: Garti N, McClements DJ, (editors). Encapsulation Technologies and Delivery Systems for Food Ingredients and Nutraceuticals. Oxford, U.K.: Woodhead Publishing; 2012. Chapter Chapter 5. p. 110-30.

[41] Ching SH, Bansal N, Bhandari B. Physical stability of emulsion encapsulated in alginate microgel particles by the impinging aerosol technique. Food Research International. 2015;75:182-93.

[42] Zeeb B, Fischer L, Weiss J. Stabilization of food dispersions by enzymes. Food \& Function. 2014;5:198-213.

[43] Guo J, Zhang Y, Yang XQ. A novel enzyme cross-linked gelation method for preparing food globular protein-based transparent hydrogel. Food Hydrocolloids. 2012;26:277-85.

[44] Karim AA, Bhat R. Gelatin alternatives for the food industry: recent developments, challenges and prospects. Trends in Food Science \& Technology. 2008;19:644-56.

[45] Foegeding EA. Food biophysics of protein gels: A challenge of nano and macroscopic proportions. Food Biophysics. 2006;1:41-50.

[46] Chen LY, Remondetto GE, Subirade M. Food protein-based materials as nutraceutical delivery systems. Trends in Food Science \& Technology. 2006;17:272-83. [47] Oxley JD. Coextrusion for food ingredients and nutraceutical encapsulation: principles and technologies. In: Garti N, McClements DJ, (editors). Encapsulation Technologies and Delivery Systems for Food Ingredients and Nutraceuticals. Oxford, U.K.: Woodhead Publishing; 2012. Chapter Chapter 6. p. 131-49.

[48] Li Y, Hu M, Du Y, Xiao H, McClements DJ. Control of lipase digestibility of emulsified lipids by encapsulation within calcium alginate beads. Food Hydrocolloids. 2011;25:122-30.

[49] Zhang ZP, Zhang RJ, Zou LQ, Chen L, Ahmed Y, Al Bishri W, et al. Encapsulation of curcumin in polysaccharide-based hydrogel beads: Impact of bead type on lipid digestion and curcumin bioaccessibility. Food Hydrocolloids. 2016;58:160-70. [50] Zhang ZP, Zhang RJ, Zou LQ, McClements DJ. Tailoring lipid digestion profiles using combined delivery systems: mixtures of nanoemulsions and filled hydrogel beads. Rsc Advances. 2016;6:65631-7.

[51] Silva CM, Ribeiro AJ, Figueiredo IV, Goncalves AR, Veiga F. Alginate microspheres prepared by internal gelation: Development and effect on insulin stability. International Journal of Pharmaceutics. 2006;311:1-10.

[52] Stojanovic R, Belscak-Cvitanovic A, Manojlovic V, Komes D, Nedovic V, Bugarski B. Encapsulation of thyme (Thymus serpyllum L.) aqueous extract in calcium alginate beads. Journal of the Science of Food and Agriculture. 2012;92:685-96.

[53] Petzold G, Gianelli MP, Buguneo G, Celan R, Pavez C, Orellana P. Encapsulation of liquid smoke flavoring in ca-alginate and ca-alginate-chitosan beads. Journal of Food Science and Technology-Mysore. 2014;51:183-90. 
[54] Zeeb B, Saberi AH, Weiss J, McClements DJ. Formation and characterization of filled hydrogel beads based on calcium alginate: Factors influencing nanoemulsion retention and release. Food Hydrocolloids. 2015;50:27-36.

[55] Leong JY, Lam WH, Ho KW, Voo WP, Lee MFX, Lim HP, et al. Advances in fabricating spherical alginate hydrogels with controlled particle designs by ionotropic gelation as encapsulation systems. Particuology. 2016;24:44-60.

[56] Zhang Z, Zhang R, Chen L, McClements DJ. Encapsulation of lactase ( $\beta$ galactosidase) into $\kappa$-carrageenan-based hydrogel beads: Impact of environmental conditions on enzyme activity. Food chemistry. 2016;200:69-75.

[57] Fang Z, Bhandari B. Spray drying, freeze drying, and related processes for food ingredient and nutraceutical encapsulation. In: Garti N, McClements DJ, (editors).

Encapsulation Technologies and Delivery Systems for Food Ingredients and Nutraceuticals. Oxford, U.K.: Woodhead Publishing; 2012. Chapter Chapter 4. p. 73-108. [58] Strobel SA, Scher HB, Nitin N, Jeoh T. In situ cross-linking of alginate during spray-drying to microencapsulate lipids in powder. Food Hydrocolloids. 2016;58:141-9. [59] Jafari SM, Assadpoor E, He YH, Bhandari B. Encapsulation efficiency of food flavours and oils during spray drying. Drying Technology. 2008;26:816-35.

[60] Vega C, Roos YH. Invited review: Spray-dried dairy and dairy-like - emulsions compositional considerations. Journal of Dairy Science. 2006;89:383-401.

[61] Desmarais SM, Haagsman HP, Barron AE. Microfabricated devices for biomolecule encapsulation. Electrophoresis. 2012;33:2639-49.

[62] Helgeson ME, Chapin SC, Doyle PS. Hydrogel microparticles from lithographic processes: Novel materials for fundamental and applied colloid science. Current Opinion in Colloid \& Interface Science. 2011;16:106-17.

[63] Selimovic S, Oh J, Bae H, Dokmeci M, Khademhosseini A. Microscale Strategies for Generating Cell-Encapsulating Hydrogels. Polymers. 2012;4:1554-79.

[64] Bjornmalm M, Yan Y, Caruso F. Engineering and evaluating drug delivery particles in microfluidic devices. Journal of Controlled Release. 2014;190:139-49.

[65] Bazban-Shotorbani S, Dashtimoghadam E, Karkhaneh A, Hasani-Sadrabadi MM, Jacob KI. Microfluidic Directed Synthesis of Alginate Nanogels with Tunable Pore Size for Efficient Protein Delivery. Langmuir. 2016;32:4996-5003.

[66] Hati AG, Bassett DC, Ribe JM, Sikorski P, Weitz DA, Stokke BT. Versatile, cell and chip friendly method to gel alginate in microfluidic devices. Lab on a Chip. 2016;16:3718-27.

[67] Marquis M, Davy J, Cathala B, Fang A, Renard D. Microfluidics assisted generation of innovative polysaccharide hydrogel microparticles. Carbohydrate Polymers.

2015;116:189-99.

[68] Utech S, Prodanovic R, Mao AS, Ostafe R, Mooney DJ, Weitz DA. Microfluidic Generation of Monodisperse, Structurally Homogeneous Alginate Microgels for Cell Encapsulation and 3D Cell Culture. Advanced Healthcare Materials. 2015;4:1628-33. [69] Pravinata L, Akhtar M, Bentley PJ, Mahatnirunkul T, Murray BS. Preparation of alginate microgels in a simple one step process via the Leeds Jet Homogenizer. Food Hydrocolloids. 2016;61:77-84.

[70] Liu H, Wang C, Gao Q, Liu X, Tong Z. Fabrication of novel core-shell hybrid alginate hydrogel beads. International Journal of Pharmaceutics. 2008;351:104-12. 
[71] Sung M-R, Xiao H, Decker EA, McClements DJ. Fabrication, characterization and properties of filled hydrogel particles formed by the emulsion-template method. Journal of Food Engineering. 2015;155:16-21.

[72] Betz M, Kulozik U. Microencapsulation of bioactive bilberry anthocyanins by means of whey protein gels. In: Saravacos G, Taoukis P, Krokida M, Karathanos V, Lazarides H, Stoforos N, et al., (editors). 11th International Congress on Engineering and Food. Vol. 12011. p. 2047-56.

[73] Saberi AH, McClements DJ. Fabrication of protein nanoparticles and microparticles within water domains formed in surfactant-oil-water mixtures: Phase inversion temperature method. Food Hydrocolloids. 2015;51:441-8.

[74] Zhai P, Chen XB, Schreyer DJ. Preparation and characterization of alginate microspheres for sustained protein delivery within tissue scaffolds. Biofabrication. $2013 ; 5$.

[75] Bulut E, Sanli O. Optimization of Release Conditions of Alzheimer's Drug Donepezil Hydrochloride from Sodium Alginate/Sodium Carboxymethyl Cellulose Blend Microspheres. Journal of Macromolecular Science Part B-Physics. 2014;53:902-17. [76] Bulut E, Sanli O. Delivery of Alzheimer's Drug Donepezil Hydrochloride from Ionically Crosslinked Alginate Microspheres Prepared by Water-in-oil Emulsion Technique: Optimization of Release Conditions. Asian Journal of Chemistry. 2013;25:3993-4000.

[77] Bulut E, Dilek M. Development and characterization of $\mathrm{pH}$-sensitive locust bean gum-alginate microspheres for controlled release of ibuprofen. Journal of Drug Delivery Science and Technology. 2014;24:613-9.

[78] McGuigan AP, Bruzewicz DA, Glavan A, Butte M, Whitesides GM. Cell Encapsulation in Sub-mm Sized Gel Modules Using Replica Molding. Plos One. 2008;3. [79] Jung S, Yi H. Fabrication of Chitosan-Poly(ethylene glycol) Hybrid Hydrogel Microparticles via Replica Molding and Its Application toward Facile Conjugation of Biomolecules. Langmuir. 2012;28:17061-70.

[80] Malone ME, Appelqvist IAM. Gelled emulsion particles for the controlled release of lipophilic volatiles during eating. Journal of controlled release. 2003;90:227-41.

[81] Qiu C, Chen M, Yan H, Wu HK. Generation of uniformly sized alginate microparticles for cell encapsulation by using a soft-lithography approach. Advanced Materials. 2007;19:1603-+.

[82] Randall CL, Leong TG, Bassik N, Gracias DH. 3D lithographically fabricated nanoliter containers for drug delivery. Advanced Drug Delivery Reviews. 2007;59:154761.

[83] Li Y, McClements DJ. Controlling lipid digestion by encapsulation of proteinstabilized lipid droplets within alginate-chitosan complex coacervates. Food Hydrocolloids. 2011;25:1025-33.

[84] Schmitt C, Sanchez C, Desobry-Banon S, Hardy J. Structure and technofunctional properties of protein-polysaccharide complexes: A review. Crit Rev Food Sci Nutr. 1998;38:689-753.

[85] Wu BC, McClements DJ. Functional hydrogel microspheres: Parameters affecting electrostatic assembly of biopolymer particles fabricated from gelatin and pectin. Food Research International. 2015;72:231-40. 
[86] Zhang Z, Zhang R, Zou L, McClements DJ. Protein encapsulation in alginate hydrogel beads: Effect of $\mathrm{pH}$ on microgel stability, protein retention and protein release. Food Hydrocolloids. 2016;58:308-15.

[87] Matalanis A, McClements DJ. Factors Influencing the Formation and Stability of Filled Hydrogel Particles Fabricated by Protein/Polysaccharide Phase Separation and Enzymatic Cross-Linking. Food Biophysics. 2012;7:72-83.

[88] Ron N, Zimet P, Bargarum J, Livney YD. Beta-lactoglobulin-polysaccharide complexes as nanovehicles for hydrophobic nutraceuticals in non-fat foods and clear beverages. International Dairy Journal. 2010;20:686-93.

[89] Comunian TA, Thomazini M, Gouvea Alves AJ, de Matos Junior FE, de Carvalho Balieiro JC, Favaro-Trindade CS. Microencapsulation of ascorbic acid by complex coacervation: Protection and controlled release. Food Research International. 2013;52:373-9.

[90] Wang B, Vongsuiuut J, Adhikari B, Barrow CJ. Microencapsulation of tuna oil fortified with the multiple lipophilic ingredients vitamins A, D-3,D- E, K-2, curcumin and coenzyme Q(10). Journal of Functional Foods. 2015;19:893-901.

[91] Hosseini SMH, Emam-Djomeh Z, Sabatino P, Van der Meeren P. Nanocomplexes arising from protein-polysaccharide electrostatic interaction as a promising carrier for nutraceutical compounds. Food Hydrocolloids. 2015;50:16-26.

[92] Gomez-Estaca J, Comunian TA, Montero P, Ferro-Furtado R, Favaro-Trindade CS. Encapsulation of an astaxanthin-containing lipid extract from shrimp waste by complex coacervation using a novel gelatin-cashew gum complex. Food Hydrocolloids. 2016;61:155-62.

[93] Jain A, Thakur D, Ghoshal G, Katare OP, Shivhare US. Characterization of microcapsulated beta-carotene formed by complex coacervation using casein and gum tragacanth. International Journal of Biological Macromolecules. 2016;87:101-13. [94] Arroyo-Maya IJ, McClements DJ. Biopolymer nanoparticles as potential delivery systems for anthocyanins: Fabrication and properties. Food Research International. 2015;69:1-8.

[95] Ben Amara C, Eghbal N, Degraeve P, Gharsallaoui A. Using complex coacervation for lysozyme encapsulation by spray-drying. Journal of Food Engineering. 2016;183:507.

[96] Calderon-Oliver M, Pedroza-Islas R, Escalona-Buendia HB, Pedraza-Chaverri J, Ponce-Alquicira E. Comparative study of the microencapsulation by complex coacervation of nisin in combination with an avocado antioxidant extract. Food Hydrocolloids. 2017;62:49-57.

[97] Matalanis A, Decker EA, McClements DJ. Inhibition of lipid oxidation by encapsulation of emulsion droplets within hydrogel microspheres. Food Chemistry. 2012;132:766-72.

[98] Kaushik P, Dowling K, McKnight S, Barrow CJ, Adhikari B. Microencapsulation of flaxseed oil in flaxseed protein and flaxseed gum complex coacervates. Food Research International. 2016;86:1-8.

[99] Koupantsis T, Pavlidou E, Paraskevopoulou A. Flavour encapsulation in milk proteins - CMC coacervate-type complexes. Food Hydrocolloids. 2014;37:134-42. 
[100] Lv Y, Yang F, Li X, Zhang X, Abbas S. Formation of heat-resistant nanocapsules of jasmine essential oil via gelatin/gum arabic based complex coacervation. Food Hydrocolloids. 2014;35:305-14.

[101] Yang Z, Peng Z, Li J, Li S, Kong L, Li P, et al. Development and evaluation of novel flavour microcapsules containing vanilla oil using complex coacervation approach. Food Chemistry. 2014;145:272-7.

[102] Frith WJ. Mixed biopolymer aqueous solutions - phase behaviour and rheology. Advances in Colloid and Interface Science. 2010;161:48-60.

[103] Norton IT, Frith WJ. Microstructure design in mixed biopolymer composites. Food Hydrocolloids. 2001;15:543-53.

[104] Matalanis A, Lesmes U, Decker EA, McClements DJ. Fabrication and characterization of filled hydrogel particles based on sequential segregative and aggregative biopolymer phase separation. Food hydrocolloids. 2010;24:689-701. [105] Matalanis A, McClements DJ. Impact of Encapsulation Within Hydrogel Microspheres on Lipid Digestion: An In Vitro Study. Food Biophysics. 2012;7:145-54. [106] Matalanis A, McClements DJ. Hydrogel microspheres for encapsulation of lipophilic components: Optimization of fabrication \& performance. Food Hydrocolloids. 2013;31:15-25.

[107] Joye IJ, McClements DJ. Production of nanoparticles by anti-solvent precipitation for use in food systems. Trends in Food Science \& Technology. 2013;34:109-23. [108] Chan HK, Kwok PCL. Production methods for nanodrug particles using the bottom-up approach. Advanced Drug Delivery Reviews. 2011;63:406-16.

[109] Thorat AA, Dalvi SV. Liquid antisolvent precipitation and stabilization of nanoparticles of poorly water soluble drugs in aqueous suspensions: Recent developments and future perspective. Chemical Engineering Journal. 2012;181:1-34. [110] Chen J, Zheng J, McClements DJ, Xiao H. Tangeretin-loaded protein nanoparticles fabricated from zein/beta-lactoglobulin: Preparation, characterization, and functional performance. Food Chemistry. 2014;158:466-72.

[111] Davidov-Pardo G, Perez-Ciordia S, Marin-Arroyo MR, McClements DJ. Improving Resveratrol Bioaccessibility Using Biopolymer Nanoparticles and Complexes: Impact of Protein-Carbohydrate Maillard Conjugation. Journal of Agricultural and Food Chemistry. 2015;63:3915-23.

[112] Zou L, Zheng B, Zhang R, Zhang Z, Liu W, Liu C, et al. Food-grade nanoparticles for encapsulation, protection and delivery of curcumin: comparison of lipid, protein, and phospholipid nanoparticles under simulated gastrointestinal conditions. Rsc Advances. 2016;6:3126-36.

[113] de Paz E, Martin A, Bartolome A, Largo M, Jose Cocero M. Development of water-soluble beta-carotene formulations by high-temperature, high-pressure emulsification and antisolvent precipitation. Food Hydrocolloids. 2014;37:14-24. [114] Zou L, Zheng B, Zhang R, Zhang Z, Liu W, Liu C, et al. Enhancing the bioaccessibility of hydrophobic bioactive agents using mixed colloidal dispersions: Curcumin-loaded zein nanoparticles plus digestible lipid nanoparticles. Food Research International. 2016;81:74-82.

[115] Huang X, Huang X, Gong Y, Xiao H, McClements DJ, Hu K. Enhancement of curcumin water dispersibility and antioxidant activity using core-shell proteinpolysaccharide nanoparticles. Food Research International. 2016;87:1-9. 
[116] de Paz E, Martin A, Every H, Jose Cocero M. Production of water-soluble quercetin formulations by antisolvent precipitation and supercritical drying. Journal of Supercritical Fluids. 2015;104:281-90.

[117] Davidov-Pardo G, Joye IJ, McClements DJ. Encapsulation of resveratrol in biopolymer particles produced using liquid antisolvent precipitation. Part 1: Preparation and characterization. Food Hydrocolloids. 2015;45:309-16.

[118] Joye IJ, Davidov-Pardo G, McClements DJ. Encapsulation of resveratrol in biopolymer particles produced using liquid antisolvent precipitation. Part 2: Stability and functionality. Food Hydrocolloids. 2015;49:127-34.

[119] Wolf B, Frith WJ, Norton IT. Morphology control in disperse biopolymer mixtures with at least one gelling component. In: Williams PA, Phillips GO, (editors). Gums and Stabilisers for the Food Industry 112002. p. 112-9.

[120] Wolf B, Frith WJ, Norton IT. Influence of gelation on particle shape in sheared biopolymer blends. Journal of Rheology. 2001;45:1141-57.

[121] Garrec DA, Norton IT. Understanding fluid gel formation and properties. Journal of Food Engineering. 2012;112:175-82.

[122] Wolf B, Frith WJ, Singleton S, Tassieri M, Norton IT. Shear behaviour of biopolymer suspensions with spheroidal and cylindrical particles. Rheologica Acta. 2001;40:238-47.

[123] Siepmann J, Siepmann F. Mathematical modeling of drug delivery. International Journal of Pharmaceutics. 2008;364:328-43.

[124] Siepmann J, Siepmann F. Modeling of diffusion controlled drug delivery. Journal of Controlled Release. 2012;161:351-62.

[125] Phawaphuthanon N, Behnam S, Koo SY, Pan CH, Chung D. Characterization of core-shell calcium-alginate macrocapsules fabricated by electro-coextrusion.

International Journal of Biological Macromolecules. 2014;65:267-74.

[126] Li XY, Kong XY, Shi SA, Zheng XL, Guo G, Wei YQ, et al. Preparation of alginate coated chitosan microparticles for vaccine delivery. Bmc Biotechnology. 2008;8. [127] Taqieddin E, Amiji M. Enzyme immobilization in novel alginate-chitosan coreshell microcapsules. Biomaterials. 2004;25:1937-45.

[128] Zou LQ, Zhang ZP, Zhang RJ, Liu W, Liu CM, Xiao H, et al. Encapsulation of protein nanoparticles within alginate microparticles: Impact of $\mathrm{pH}$ and ionic strength on functional performance. Journal of Food Engineering. 2016;178:81-9.

[129] Schmitt C, Moitzi C, Bovay C, Rouvet M, Bovetto L, Donato L, et al. Internal structure and colloidal behaviour of covalent whey protein microgels obtained by heat treatment. Soft Matter. 2010;6:4876-84.

[130] McClements DJ. Theoretical prediction of emulsion color. Advances in Colloid and Interface Science. 2002;97:63-89.

[131] Lin CC, Metters AT. Hydrogels in controlled release formulations: Network design and mathematical modeling. Advanced Drug Delivery Reviews. 2006;58:1379-408.

[132] Amsden B. Solute diffusion within hydrogels. Mechanisms and models.

Macromolecules. 1998;31:8382-95.

[133] Peppas NA, Bures P, Leobandung W, Ichikawa H. Hydrogels in pharmaceutical formulations. European Journal of Pharmaceutics and Biopharmaceutics. 2000;50:27-46. 
[134] Zeeb B, Grossmann L, Weiss J. Accessibility of Transglutaminase to Induce Protein Crosslinking in Gelled Food Matrices - Impact of Membrane Structure. Food Biophysics. 2016;11:176-83.

[135] Zhang ZP, Zhang RJ, Zou LQ, McClements DJ. Protein encapsulation in alginate hydrogel beads: Effect of $\mathrm{pH}$ on microgel stability, protein retention and protein release. Food Hydrocolloids. 2016;58:308-15.

[136] Crcarevska MS, Dodov MG, Goracinova K. Chitosan coated Ca-alginate microparticles loaded with budesonide for delivery to the inflamed colonic mucosa. European Journal of Pharmaceutics and Biopharmaceutics. 2008;68:565-78.

[137] Choi CH, Wang HN, Lee H, Kim JH, Zhang LY, Mao A, et al. One-step generation of cell-laden microgels using double emulsion drops with a sacrificial ultra-thin oil shell.

Lab on a Chip. 2016;16:1549-55.

[138] Qian C, Decker EA, Xiao H, McClements DJ. Physical and chemical stability of beta-carotene-enriched nanoemulsions: Influence of $\mathrm{pH}$, ionic strength, temperature, and emulsifier type. Food Chemistry. 2012;132:1221-9.

[139] Heger M, van Golen RF, Broekgaarden M, Michel MC. The Molecular Basis for the Pharmacokinetics and Pharmacodynamics of Curcumin and Its Metabolites in Relation to Cancers. Pharmacological Reviews. 2014;66:222-307. [140] Adroher-Benitez I, Ahualli S, Martin-Molina A, Quesada-Perez M, Moncho-Jorda A. Role of Steric Interactions on the Ionic Permeation Inside Charged Microgels: Theory and Simulations. Macromolecules. 2015;48:4645-56.

[141] Zhang Z, Chen F, Zhang R, Deng Z, McClements DJ. Encapsulation of pancreatic lipase in hydrogel beads with self-regulating internal $\mathrm{pH}$ microenvironments: Retention of lipase activity after exposure to gastric conditions. Journal of Agricultural and Food Chemistry. 2017; In Press.

[142] Zeeb B, Saberi AH, Weiss J, McClements DJ. Retention and release of oil-in-water emulsions from filled hydrogel beads composed of calcium alginate: impact of emulsifier type and pH. Soft Matter. 2015;11:2228-36.

[143] Nuchi CD, Hernandez P, McClements DJ, Decker EA. Ability of lipid hydroperoxides to partition into surfactant micelles and alter lipid oxidation rates in emulsions. Journal of Agricultural and Food Chemistry. 2002;50:5445-9. [144] McClements DJ. Colloidal basis of emulsion color. Current Opinion in Colloid \& Interface Science. 2002;7:451-5. [145] Chung C, Degner B, McClements DJ. Development of Reduced-calorie foods: Microparticulated whey proteins as fat mimetics in semi-solid food emulsions. Food Research International. 2014;56:136-45.

[146] Chung C, McClements DJ. Controlling microstructure and physical properties of biopolymer hydrogel particles through modulation of electrostatic interactions. Journal of Food Engineering. 2015;158:13-21.

[147] Chung C, Degner B, McClements DJ. Reduced calorie emulsion-based foods: Protein microparticles and dietary fiber as fat replacers. Food Research International. 2014;64:664-76.

[148] Chung C, Degner B, McClements DJ. Physicochemical characteristics of mixed colloidal dispersions: Models for foods containing fat and starch. Food Hydrocolloids. 2013;30:281-91. 
[149] Jonassen H, Kjoniksen AL, Hiorth M. Stability of Chitosan Nanoparticles CrossLinked with Tripolyphosphate. Biomacromolecules. 2012;13:3747-56.

[150] McClements DJ. Food Emulsions: Principles, Practices, and Techniques. Third ed: CRC press; 2015.

[151] Hunter RJ. Foundations of Colloid Science. Oxford: Oxford University Press; 1989.

[152] Ching SH, Bansal N, Bhandari B. Rheology of emulsion-filled alginate microgel suspensions. Food Research International. 2016;80:50-60.

[153] Davidov-Pardo G, Joye IJ, Espinal-Ruiz M, McClements DJ. Effect of Maillard Conjugates on the Physical Stability of Zein Nanoparticles Prepared by Liquid

Antisolvent Coprecipitation. Journal of Agricultural and Food Chemistry. 2015;63:85108.

[154] Jones OG, Decker EA, McClements DJ. Comparison of protein-polysaccharide nanoparticle fabrication methods: Impact of biopolymer complexation before or after particle formation. Journal of Colloid and Interface Science. 2010;344:21-9.

[155] Gonzalez-Mozuelos P. Effective electrostatic interactions among charged thermoresponsive microgels immersed in a simple electrolyte. Journal of Chemical Physics. $2016 ; 144$.

[156] Patel AR, Bouwens ECM, Velikov KP. Sodium Caseinate Stabilized Zein Colloidal Particles. Journal of Agricultural and Food Chemistry. 2010;58:12497-503.

[157] Osborne JL, Farmer R, Woodhouse KA. Self-assembled elastin-like polypeptide particles. Acta Biomaterialia. 2008;4:49-57.

[158] Sanchez C, Mekhloufi G, Schmitt C, Renard D, Robert P, Lehr CM, et al. Selfassembly of beta-lactoglobulin and acacia gum in aqueous solvent: Structure and phaseordering kinetics. Langmuir. 2002;18:10323-33.

[159] McClements DJ. Food Emulsions: Principles, Practice, and Techniques. 2nd ed. Boca Raton: CRC Press; 2005.

[160] Lekka M, Sainz-Serp D, Kulik AJ, Wandrey C. Hydrogel microspheres: Influence of chemical composition on surface morphology, local elastic properties, and bulk mechanical characteristics. Langmuir. 2004;20:9968-77.

[161] Gray A, Egan S, Bakalis S, Zhang ZB. Determination of microcapsule physicochemical, structural, and mechanical properties. Particuology. 2016;24:32-43. [162] Voo WP, Ooi CW, Islam A, Tey BT, Chan ES. Calcium alginate hydrogel beads with high stiffness and extended dissolution behaviour. European Polymer Journal. 2016;75:343-53.

[163] Neubauer MP, Poehlmann M, Fery A. Microcapsule mechanics: From stability to function. Advances in Colloid and Interface Science. 2014;207:65-80.

[164] Genovese DB, Lozano JE, Rao MA. The rheology of colloidal and noncolloidal food dispersions. Journal of Food Science. 2007;72:R11-R20.

[165] Pal R. Rheology of simple and multiple emulsions. Current Opinion in Colloid \& Interface Science. 2011;16:41-60.

[166] Tadros TF. Fundamental principles of emulsion rheology and their applications. Colloids and Surfaces a-Physicochemical and Engineering Aspects. 1994;91:39-55. [167] Shewan HM, Stokes JR. Viscosity of soft spherical micro-hydrogel suspensions. Journal of Colloid and Interface Science. 2015;442:75-81. 
[168] Eichenbaum GM, Kiser PF, Dobrynin AV, Simon SA, Needham D. Investigation of the swelling response and loading of ionic microgels with drugs and proteins: The dependence on cross-link density. Macromolecules. 1999;32:4867-78.

[169] Chan AW, Neufeld RJ. Modeling the controllable pH-responsive swelling and pore size of networked alginate based biomaterials. Biomaterials. 2009;30:6119-29.

[170] Farr RS. Random close packing fractions of lognormal distributions of hard spheres. Powder Technology. 2013;245:28-34.

[171] Farr RS, Groot RD. Close packing density of polydisperse hard spheres. Journal of Chemical Physics. 2009;131.

[172] Pellet C, Cloitre M. The glass and jamming transitions of soft polyelectrolyte microgel suspensions. Soft Matter. 2016;12:3710-20.

[173] Engelen L, Van der Bilt A, Schipper M, Bosman F. Oral size perception of particles: Effect of size, type, viscosity and method. Journal of Texture Studies. 2005;36:373-86.

[174] Engelen L, de Wijk RA, van der Bilt A, Prinz JF, Janssen AM, Bosman F. Relating particles and texture perception. Physiol Behav. 2005;86:111-7.

[175] Imai E, Shimichi Y, Maruyama I, Inoue A, Ogawa S, Hatae K, et al. Perception of grittiness in an oil-in-water emulsion. Journal of Texture Studies. 1997;28:257-72.

[176] Imai E, Saito K, Hatakeyama M, Hatae K, Shimada A. Effect of physical properties of food particles on the degree of graininess perceived in the mouth. Journal of Texture Studies. 1999;30:59-88.

[177] Liechty WB, Kryscio DR, Slaughter BV, Peppas NA. Polymers for Drug Delivery Systems. In: Prausnitz JM, Doherty MF, Segalman MA, (editors). Annual Review of Chemical and Biomolecular Engineering, Vol 1. Vol. 1. Palo Alto: Annual Reviews; 2010. p. 149-73.

[178] Peppas NA, Huang Y, Torres-Lugo M, Ward JH, Zhang J. Physicochemical foundations and structural design of hydrogels in medicine and biology. Annual review of biomedical engineering. 2000;2:9-29.

[179] Baker RW. Controlled Release of Biologically Active Agents. New York, NY.: John Wiley \& Sons; 1987.

[180] Crank J. The Mathematics of Diffusion. Second ed. Oxford, U.K.: Oxford Science Publications; 1975.

[181] Zhang Y, Amsden BG. Application of an obstruction-scaling model to diffusion of vitamin B-12 and proteins in semidilute alginate solutions. Macromolecules. 2006;39:1073-8.

[182] Grassi M, Grassi G. Application of mathematical modeling in sustained release delivery systems. Expert Opinion on Drug Delivery. 2014;11:1299-321.

[183] Siepmann J, Siepmann F. Mathematical modeling of drug dissolution. International Journal of Pharmaceutics. 2013;453:12-24.

[184] Burey P, Bhandari BR, Howes T, Gidley MJ. Gel particles from spray-dried disordered polysaccharides. Carbohydrate Polymers. 2009;76:206-13.

[185] Malmsten M, Bysell H, Hansson P. Biomacromolecules in microgels -

Opportunities and challenges for drug delivery. Current Opinion in Colloid \& Interface Science. 2010;15:435-44. 
[186] Sarkar A, Murray B, Holmes M, Ettelaie R, Abdalla A, Yang XY. In vitro digestion of Pickering emulsions stabilized by soft whey protein microgel particles: influence of thermal treatment. Soft Matter. 2016;12:3558-69.

[187] Maltais A, Remondetto GE, Subirade M. Tabletted soy protein cold-set hydrogels as carriers of nutraceutical substances. Food Hydrocolloids. 2010;24:518-24. [188] Hebrard G, Hoffart V, Cardot JM, Subirade M, Beyssac E. Development and characterization of coated-microparticles based on whey protein/alginate using the Encapsulator device. Drug Development and Industrial Pharmacy. 2013;39:128-37. [189] Maltais A, Remondetto GE, Subirade M. Soy protein cold-set hydrogels as controlled delivery devices for nutraceutical compounds. Food Hydrocolloids. 2009;23:1647-53.

[190] Liu CZ, Jiang SS, Han ZJ, Xiong L, Sun QJ. In vitro digestion of nanoscale starch particles and evolution of thermal, morphological, and structural characteristics. Food Hydrocolloids. 2016;61:344-50.

[191] Balmayor ER, Baran TE, Unger M, Marques AP, Azevedo HS, Reis RL. Presence of starch enhances in vitro biodegradation and biocompatibility of a gentamicin delivery formulation. Journal of Biomedical Materials Research Part B-Applied Biomaterials. 2015;103:1610-20.

[192] Oehlke K, Adamiuk M, Behsnilian D, Graf V, Mayer-Miebach E, Walz E, et al. Potential bioavailability enhancement of bioactive compounds using food-grade engineered nanomaterials: a review of the existing evidence. Food \& Function. 2014;5:1341-59.

[193] Klinger D, Landfester K. Stimuli-responsive microgels for the loading and release of functional compounds: Fundamental concepts and applications. Polymer.

2012;53:5209-31.

[194] Quesada-Perez M, Maroto-Centeno JA, Forcada J, Hidalgo-Alvarez R. Gel swelling theories: the classical formalism and recent approaches. Soft Matter.

2011;7:10536-47.

[195] Yang X, Kim JC. Novel pH-sensitive microgels prepared using salt bridge. International Journal of Pharmaceutics. 2010;388:58-63.

[196] Alvarez-Lorenzo C, Blanco-Fernandez B, Puga AM, Concheiro A. Crosslinked ionic polysaccharides for stimuli-sensitive drug delivery. Advanced Drug Delivery Reviews. 2013;65:1148-71.

[197] Aguilera JM. Food microstructure affects the bioavailability of several nutrients. Journal of Food Science. 2006;72:R21-R32.

[198] Kaunisto E, Marucci M, Borgquist P, Axelsson A. Mechanistic modelling of drug release from polymer-coated and swelling and dissolving polymer matrix systems. International Journal of Pharmaceutics. 2011;418:54-77.

[199] Peppas NA. Historical perspective on advanced drug delivery: How engineering design and mathematical modeling helped the field mature. Advanced Drug Delivery Reviews. 2013;65:5-9. [200] Aguzzi C, Cerezo P, Salcedo I, Sanchez R, Viseras C. Mathematical models describing drug release from biopolymeric delivery systems. Materials Technology. 2010;25:205-11. 
[201] Sackett CK, Narasimhan B. Mathematical modeling of polymer erosion: Consequences for drug delivery. International Journal of Pharmaceutics. 2011;418:10414.

[202] Arifin DY, Lee LY, Wang C-H. Mathematical modeling and simulation of drug release from microspheres: Implications to drug delivery systems. Advanced drug delivery reviews. 2006;58:1274-325.

[203] Grassi M, Grassi G. Mathematical modelling and controlled drug delivery: matrix systems. Current drug delivery. 2005;2:97-116.

[204] Hamidi M, Azadi A, Rafiei P. Hydrogel nanoparticles in drug delivery. Advanced Drug Delivery Reviews. 2008;60:1638-49.

[205] Hoare TR, Kohane DS. Hydrogels in drug delivery: progress and challenges. Polymer. 2008;49:1993-2007.

[206] Bysell H, Mansson R, Hansson P, Malmsten M. Microgels and microcapsules in peptide and protein drug delivery. Advanced Drug Delivery Reviews. 2011;63:1172-85. [207] Gombotz WR, Wee SF. Protein release from alginate matrices. Advanced Drug Delivery Reviews. 2012;64:194-205.

[208] Marquis M, Alix V, Capron I, Cuenot S, Zykwinska A. Microfluidic Encapsulation of Pickering Oil Microdroplets into Alginate Microgels for Lipophilic Compound Delivery. Acs Biomaterials Science \& Engineering. 2016;2:535-43.

[209] Jain D, Bar-Shalom D. Alginate drug delivery systems: application in context of pharmaceutical and biomedical research. Drug Development and Industrial Pharmacy. 2014;40:1576-84.

[210] Al Dalaty A, Karam A, Najlah M, Alany RG, Khoder M. Effect of non-crosslinked calcium on characteristics, swelling behaviour, drug release and mucoadhesiveness of calcium alginate beads. Carbohydrate Polymers. 2016;140:163-70. [211] Agnihotri SA, Mallikarjuna NN, Aminabhavi TM. Recent advances on chitosanbased micro- and nanoparticles in drug delivery. Journal of Controlled Release. 2004;100:5-28.

[212] Chang CY, Zhang LN. Cellulose-based hydrogels: Present status and application prospects. Carbohydrate Polymers. 2011;84:40-53.

[213] Kamel S, Ali N, Jahangir K, Shah SM, El-Gendy AA. Pharmaceutical significance of cellulose: A review. Express Polymer Letters. 2008;2:758-78.

[214] Liu LS, Fishman ML, Kost J, Hicks KB. Pectin-based systems for colon-specific drug delivery via oral route. Biomaterials. 2003;24:3333-43.

[215] Liu LS, Fishman ML, Hicks KB. Pectin in controlled drug delivery - a review. Cellulose. 2007;14:15-24.

[216] Munarin F, Tanzi MC, Petrini P. Advances in biomedical applications of pectin gels. International Journal of Biological Macromolecules. 2012;51:681-9.

[217] Sriamornsak P. Application of pectin in oral drug delivery. Expert Opinion on Drug Delivery. 2011;8:1009-23.

[218] Ismail H, Irani M, Ahmad Z. Starch-Based Hydrogels: Present Status and Applications. International Journal of Polymeric Materials and Polymeric Biomaterials. 2013;62:411-20.

[219] Alvani K, Qi X, Tester RF. Use of carbohydrates, including dextrins, for oral delivery. Starch-Starke. 2011;63:424-31. 
[220] Liu JJ, Zhan XD, Wan JB, Wang YT, Wang CM. Review for carrageenan-based pharmaceutical biomaterials: Favourable physical features versus adverse biological effects. Carbohydrate Polymers. 2015;121:27-36.

[221] Ofokansi KC, Kenechukwu FC, Isah AB, Okigbo EL. Formulation and Evaluation of Glutaraldehyde-Crosslinked Chitosan Microparticles for the Delivery of Ibuprofen. Tropical Journal of Pharmaceutical Research. 2013;12:19-25.

[222] Milasinovic N, Calija B, Vidovic B, Sakac MC, Vujic Z, Knezevic-Jugovic Z. Sustained release of alpha-lipoic acid from chitosan microbeads synthetized by inverse emulsion method. Journal of the Taiwan Institute of Chemical Engineers. 2016;60:10612.

[223] Gu Z, Dang TT, Ma ML, Tang BC, Cheng H, Jiang S, et al. Glucose-Responsive Microgels Integrated with Enzyme Nanocapsules for Closed-Loop Insulin Delivery. Acs Nano. 2013;7:6758-66.

[224] Walke S, Srivastava G, Nikalje M, Doshi J, Kumar R, Ravetkar S, et al. Fabrication of chitosan microspheres using vanillin/TPP dual crosslinkers for protein antigens encapsulation. Carbohydrate Polymers. 2015;128:188-98.

[225] Li PP, Liu RG. Cellulose Gels and Microgels: Synthesis, Service, and Supramolecular Interactions. In: Seiffert S, (editor). Supramolecular Polymer Networks and Gels. Vol. 2682015. p. 209-51.

[226] Kim S, Oh J, Cha C. Enhancing the biocompatibility of microfluidics-assisted fabrication of cell-laden microgels with channel geometry. Colloids and Surfaces BBiointerfaces. 2016;147:1-8.

[227] Knipe JM, Strong LE, Peppas NA. Enzyme- and pH-Responsive

Microencapsulated Nanogels for Oral Delivery of siRNA to Induce TNF-alpha

Knockdown in the Intestine. Biomacromolecules. 2016;17:788-97.

[228] Chen C, Liu MZ, Lu SY, Gao CM, Chen JC. In Vitro Degradation and Drug-

Release Properties of Water-Soluble Chitosan Cross-Linked Oxidized Sodium Alginate

Core-Shell Microgels. Journal of Biomaterials Science-Polymer Edition. 2012;23:200724.

[229] Zhang ZP, Zhang RJ, McClements DJ. Encapsulation of beta-carotene in alginatebased hydrogel beads: Impact on physicochemical stability and bioaccessibility. Food Hydrocolloids. 2016;61:1-10.

[230] Cujic N, Trifkovic K, Bugarski B, Ibric S, Pljevljakusic D, Savikin K. Chokeberry (Aronia melanocarpa L.) extract loaded in alginate and alginate/inulin system. Industrial Crops and Products. 2016;86:120-31.

[231] Burgain J, Gaiani C, Linder M, Scher J. Encapsulation of probiotic living cells: From laboratory scale to industrial applications. Journal of Food Engineering. 2011;104:467-83.

[232] Rokka S, Rantamaki P. Protecting probiotic bacteria by microencapsulation: challenges for industrial applications. European Food Research and Technology. 2010;231:1-12.

[233] Huq T, Khan A, Khan RA, Riedl B, Lacroix M. Encapsulation of Probiotic Bacteria in Biopolymeric System. Crit Rev Food Sci Nutr. 2013;53:909-16.

[234] Martin MJ, Lara-Villoslada F, Ruiz MA, Morales ME. Microencapsulation of bacteria: A review of different technologies and their impact on the probiotic effects. Innovative Food Science \& Emerging Technologies. 2015;27:15-25. 
[235] Solanki HK, Pawar DD, Shah DA, Prajapati VD, Jani GK, Mulla AM, et al. Development of Microencapsulation Delivery System for Long-Term Preservation of Probiotics as Biotherapeutics Agent. Biomed Research International. 2013.

[236] Li R, Zhang YF, Polk DB, Tomasula PM, Yan F, Liu LS. Preserving viability of Lactobacillus rhamnosus GG in vitro and in vivo by a new encapsulation system. Journal of Controlled Release. 2016;230:79-87.

[237] Yeung TW, Ucok EF, Tiani KA, McClements DJ, Sela DA. Microencapsulation in Alginate and Chitosan Microgels to Enhance Viability of Bifidobacterium longum for Oral Delivery. Frontiers in Microbiology. 2016;7.

[238] Yeung TW, Arroyo-Maya IJ, McClements DJ, Sela DA. Microencapsulation of probiotics in hydrogel particles: enhancing Lactococcus lactis subsp cremoris LM0230 viability using calcium alginate beads. Food \& Function. 2016;7:1797-804.

[239] Li W, Luo XG, Song R, Zhu Y, Li B, Liu SL. Porous Cellulose Microgel Particle: A Fascinating Host for the Encapsulation, Protection, and Delivery of Lactobacillus plantarum. Journal of Agricultural and Food Chemistry. 2016;64:3430-6.

[240] Mai THA, Tran VN, Le VVM. Biochemical studies on the immobilized lactase in the combined alginate-carboxymethyl cellulose gel. Biochemical Engineering Journal. 2013;74:81-7.

[241] Zhang S, Shang WT, Yang XX, Zhang SJ, Zhang XG, Chen JW. Immobilization of Lipase using Alginate Hydrogel Beads and Enzymatic Evaluation in Hydrolysis of pNitrophenol Butyrate. Bulletin of the Korean Chemical Society. 2013;34:2741-6. [242] Malone ME, Appelqvist IAM, Norton IT. Oral behaviour of food hydrocolloids and emulsions. Part 2. Taste and aroma release. Food Hydrocolloids. 2003;17:775-84. 
Figure 1: In general, the fabrication of bioactive-loaded hydrogel beads can be considered to consist of two steps that may occur simultaneously or consecutively: particle formation and particle gelation. Hydrophilic bioactives are usually directly mixed with the aqueous biopolymer solution, whereas hydrophobic nutraceuticals have to be dispersed in oil droplets first.

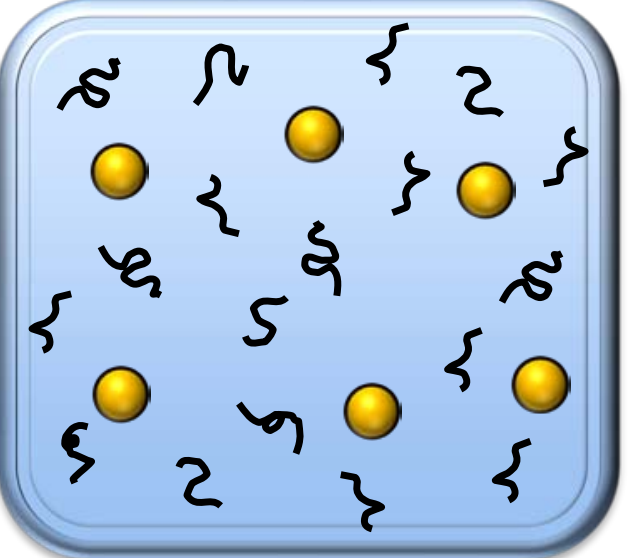

Particle Formation

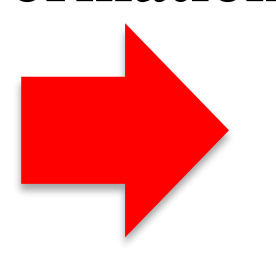

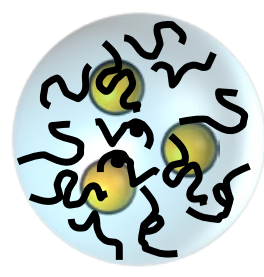

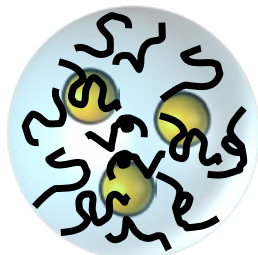

Solution of Bioactives \& Biopolymers s

\section{Particle} Gelation
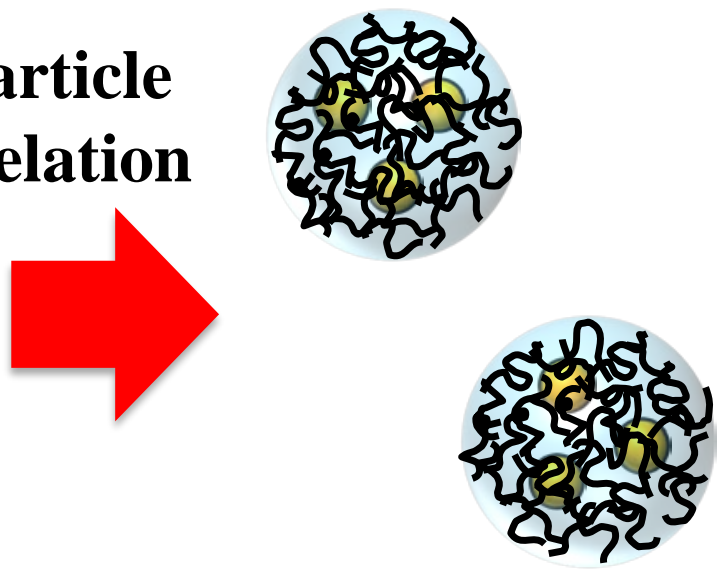

Bioactive-Loaded Hydrogel Beads 
Figure 2: Various kinds of different materials can be incorporated into microgels, including molecules, micelles, liquid droplets, solid particles, liposomes, biopolymers and structured particles.

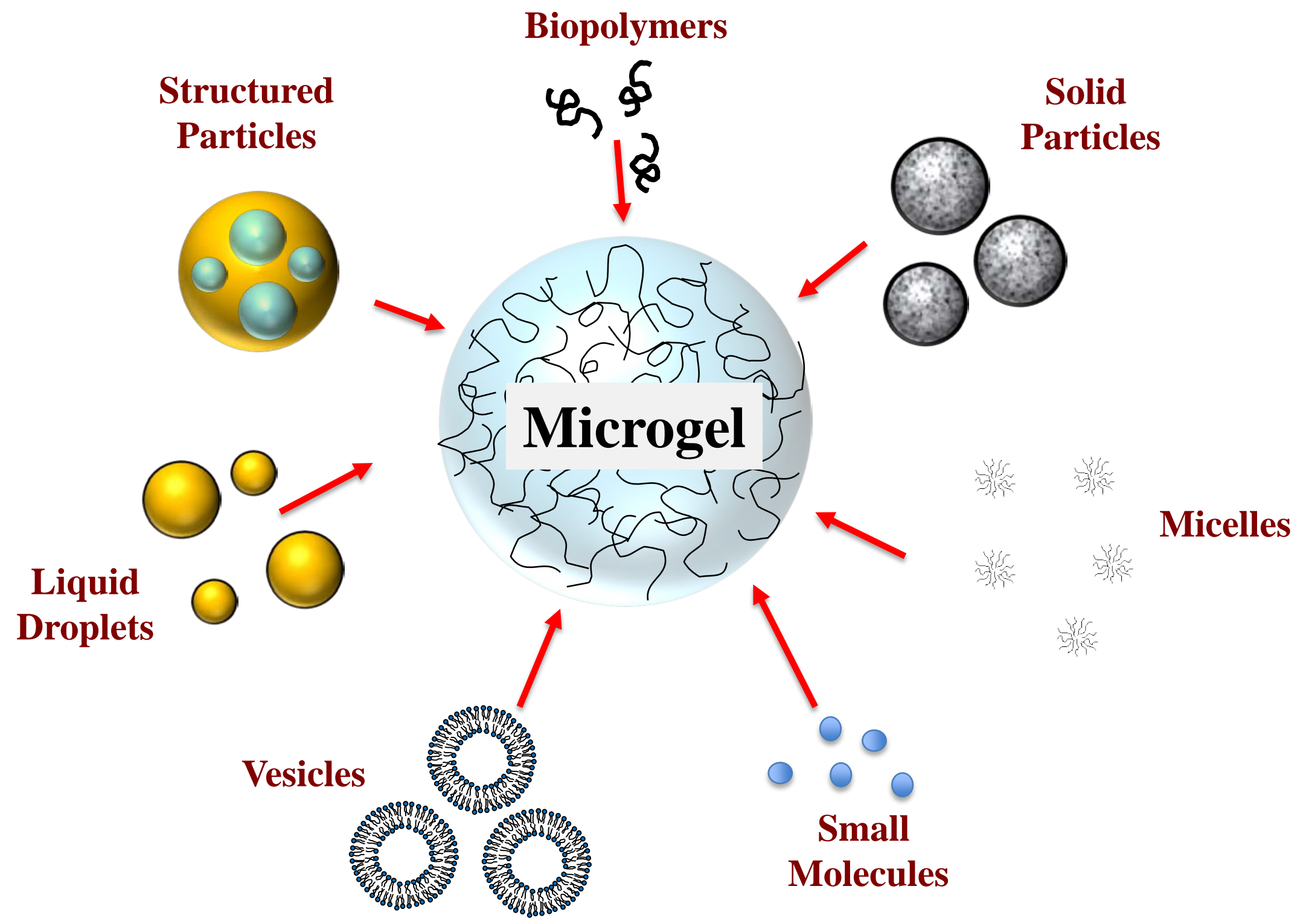


Figure 3: Example of a simple extrusion method that can be used to form bioactive-loaded hydrogel beads in the laboratory. A mixture of bioactives and biopolymers is injected into a gelling solution that initiates crosslinking of the biopolymer molecules.

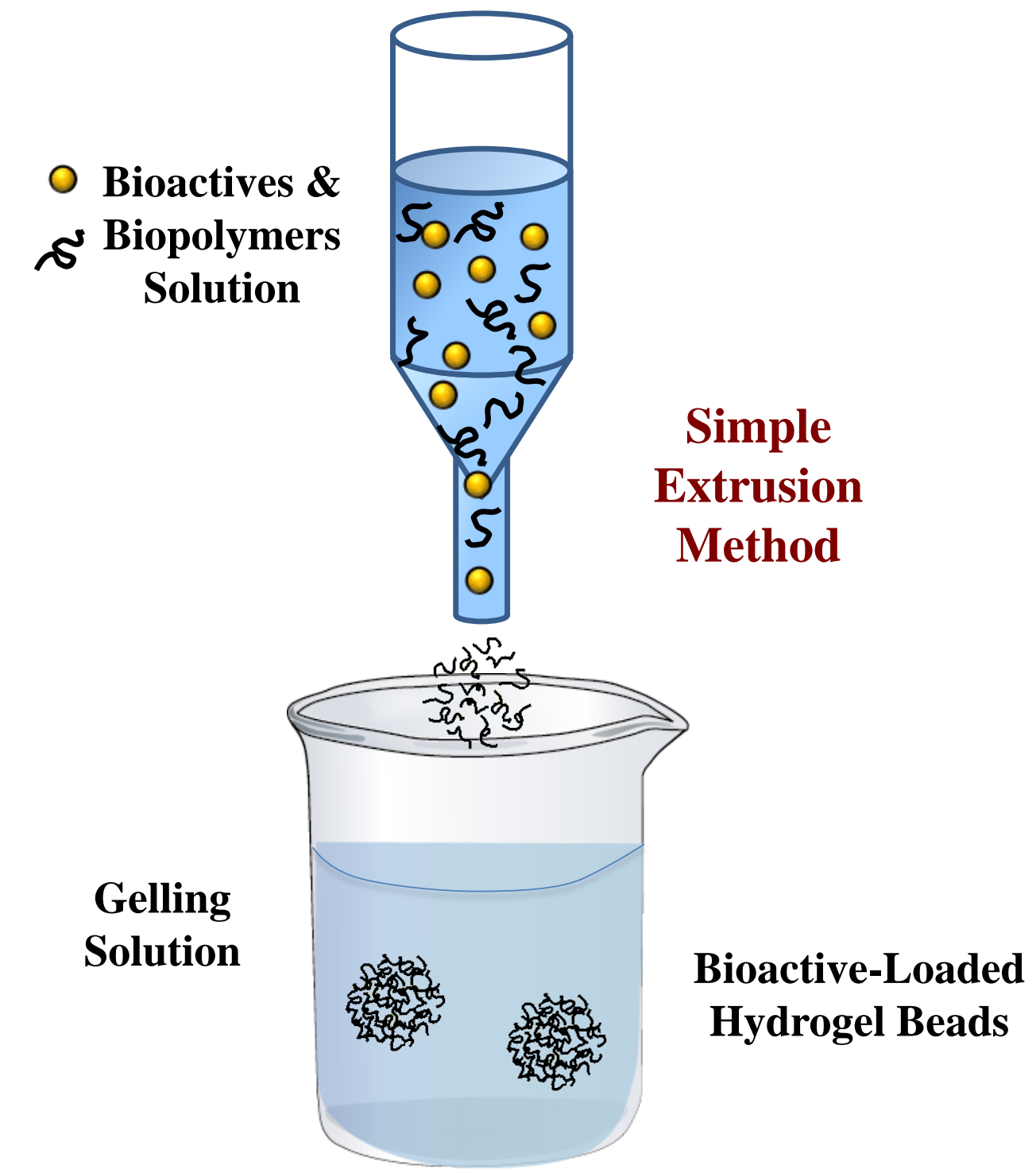


Figure 4: Example of a microfluidic method that can be used to form bioactive-loaded hydrogel beads. An aqueous solution containing bioactives and biopolymers is injected into a flowing oil phase that leads to particle formation. The environmental or solution conditions are then change to initiate cross-linking of the biopolymer molecules and cause particle gelation.

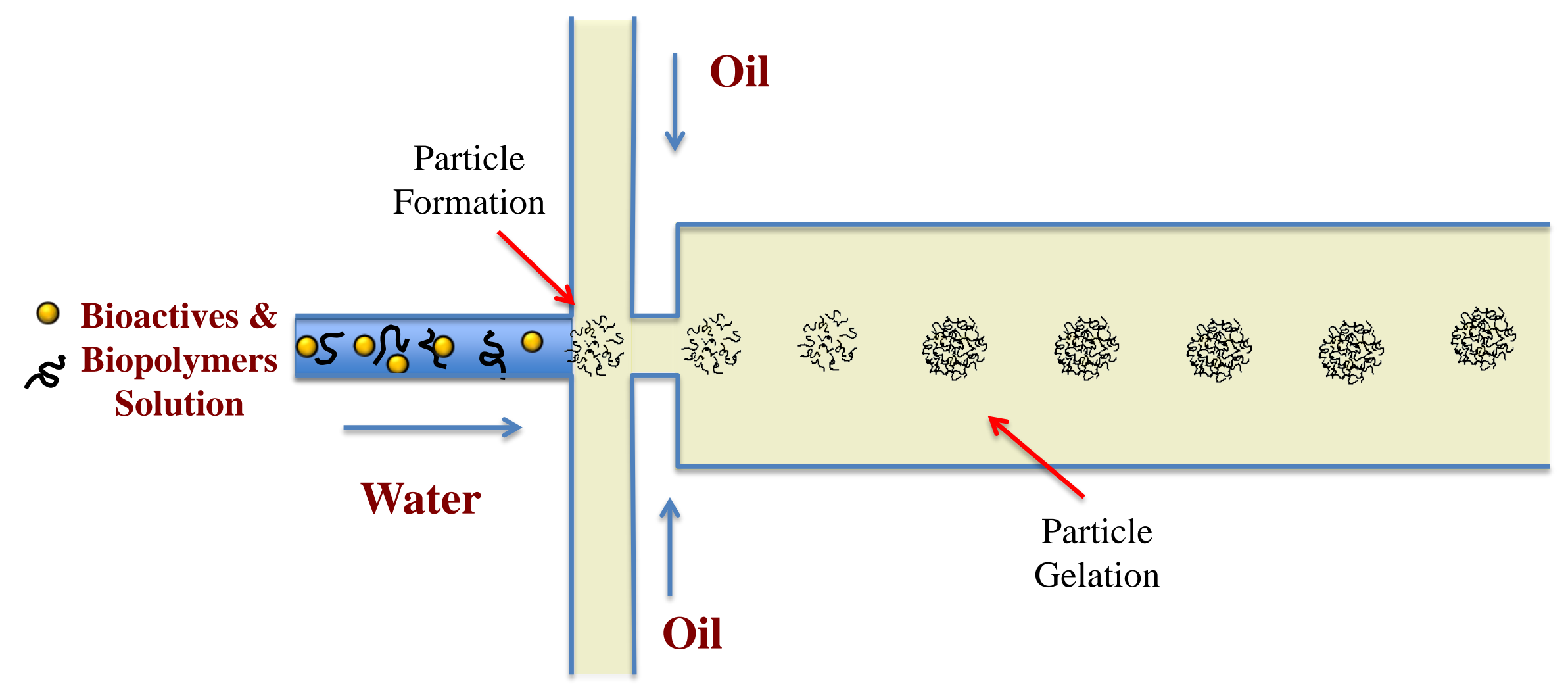




\section{Bioactives \&}

\section{Biopolymers}

\section{Solution}
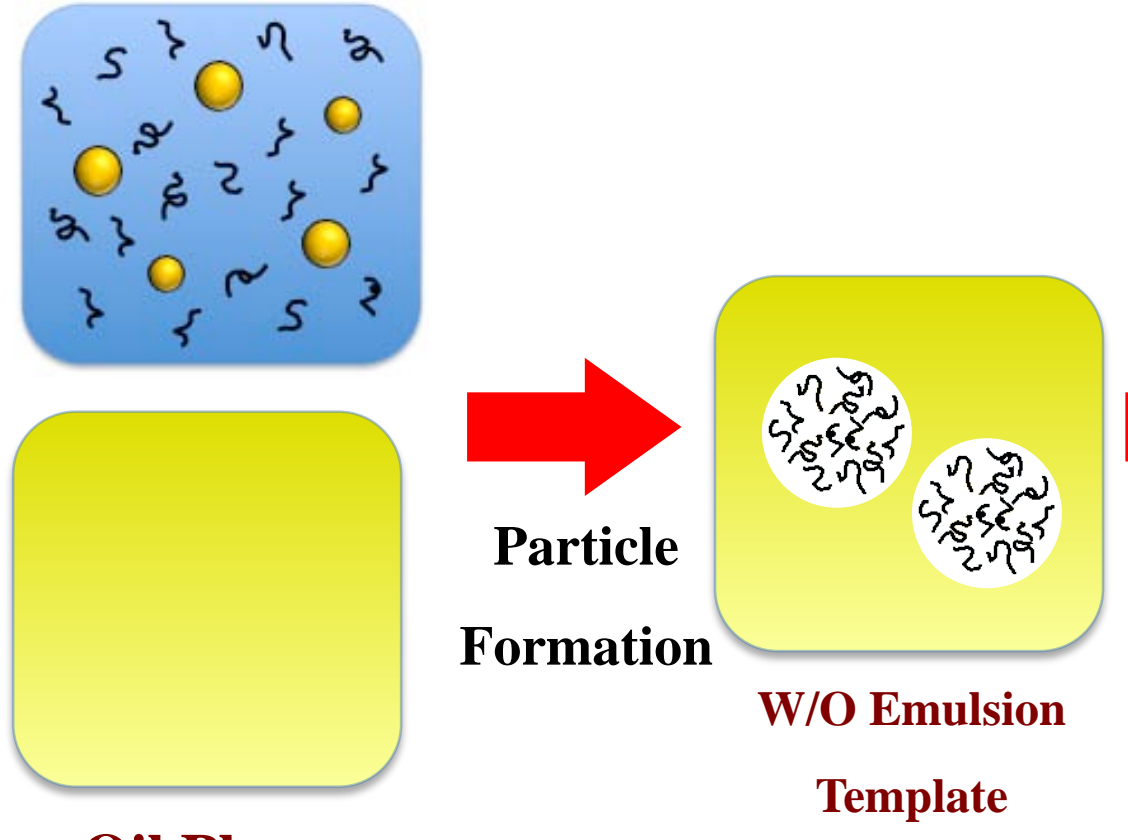

\section{Oil Phase}
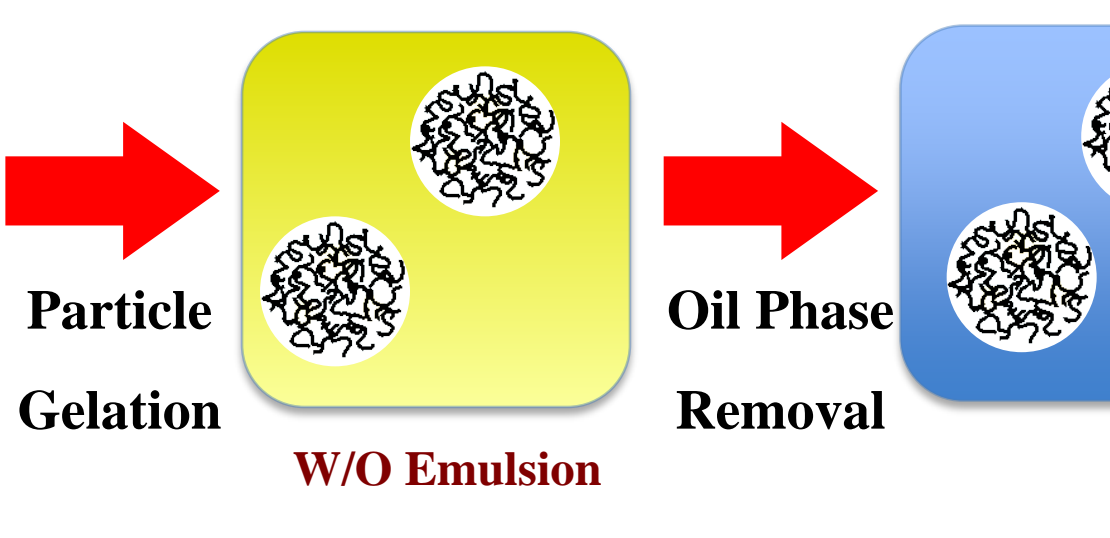

\section{Removal}

Template

\section{Microgels}




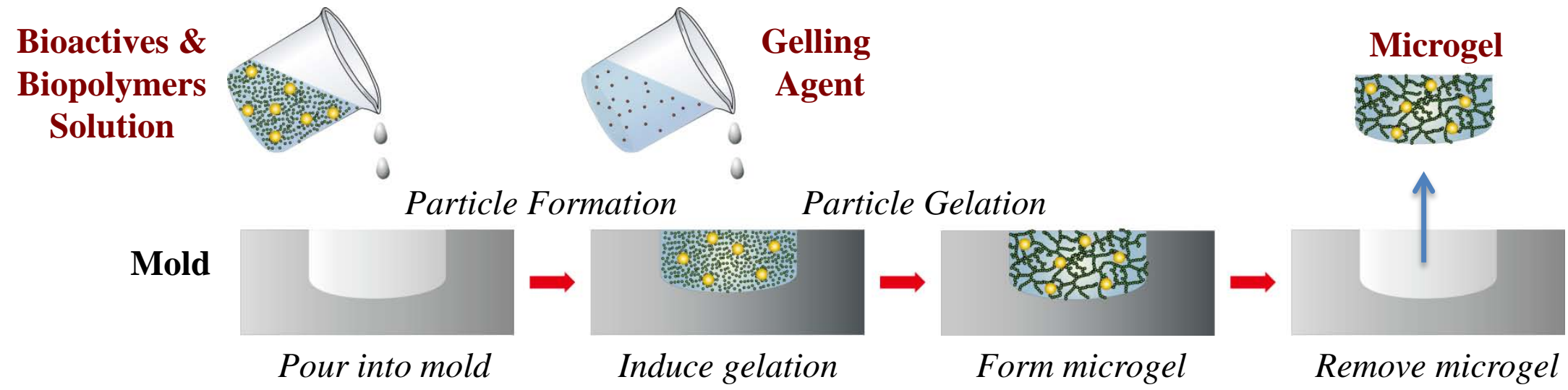

Figure 6. Schematic diagram of lithographic templating method for forming microgels. An aqueous solution containing bioactives and biopolymers is poured into a mold. The biopolymer molecules are then cross-linked by changing environmental conditions (such as temperature) or adding a gelling agent. The resulting microgels are then removed from the mold. 
Figure 7: Bioactives can be encapsulated within hydrogel beads that have different internal structures, such as homogeneous or heterogeneous (core-shell or dispersion).

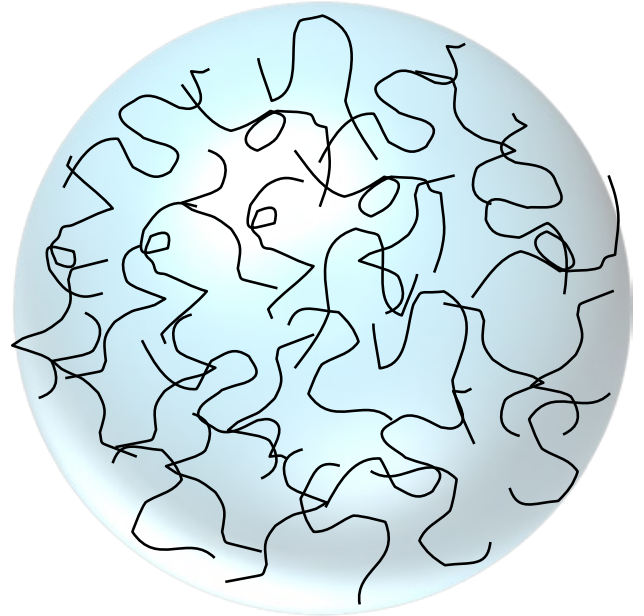

Homogeneous

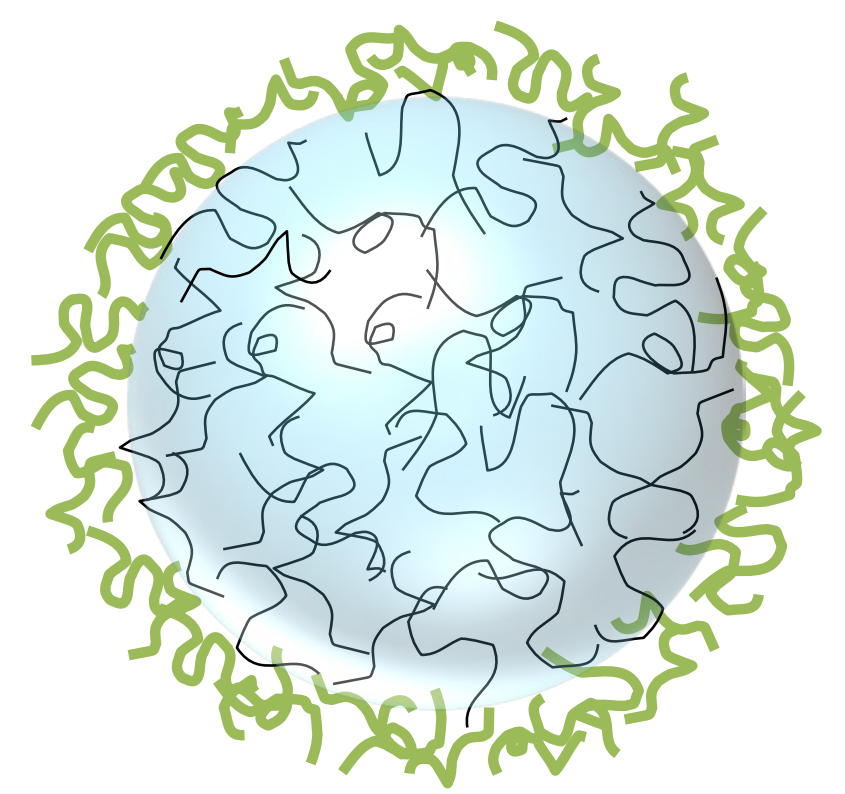

Core-Shell

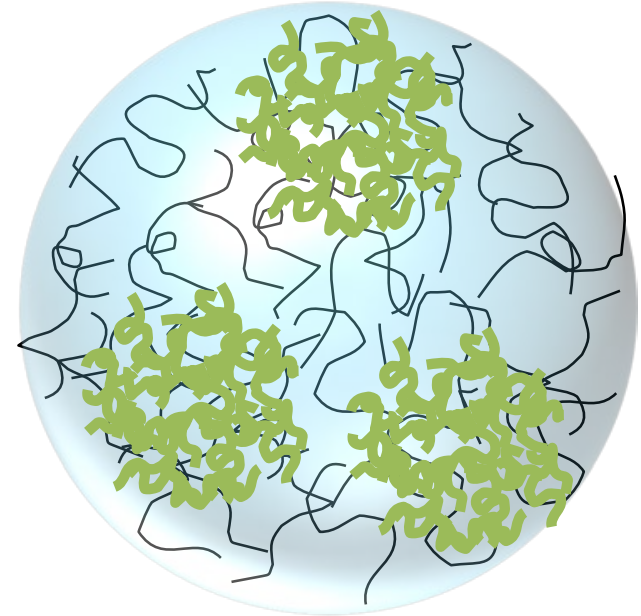

Dispersion

\section{Heterogeneous}


Figure 8: Bioactives will be retained when their dimensions are appreciably greater than the pore size of the biopolymer network inside microgels.

\section{Small Pores}

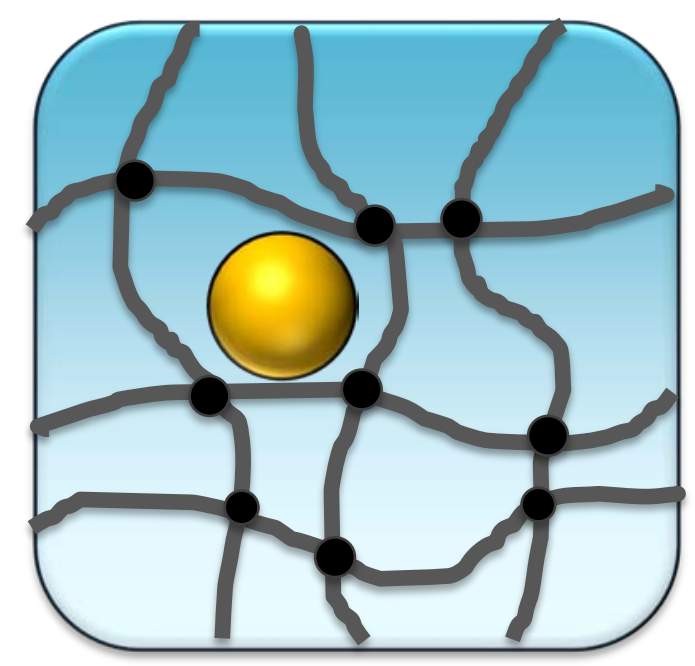

\section{Retention}

\section{Release}

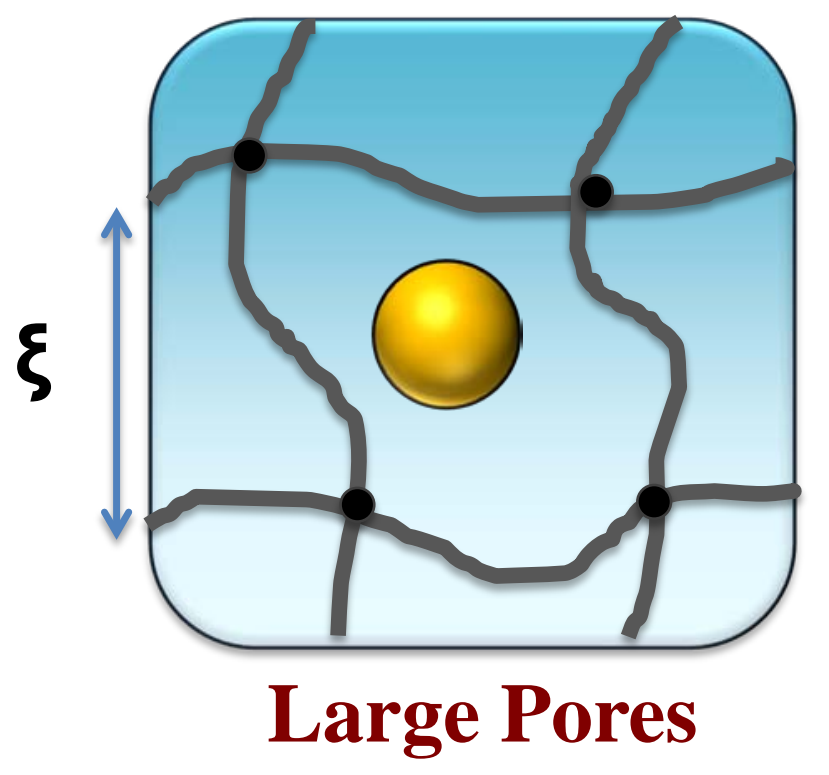

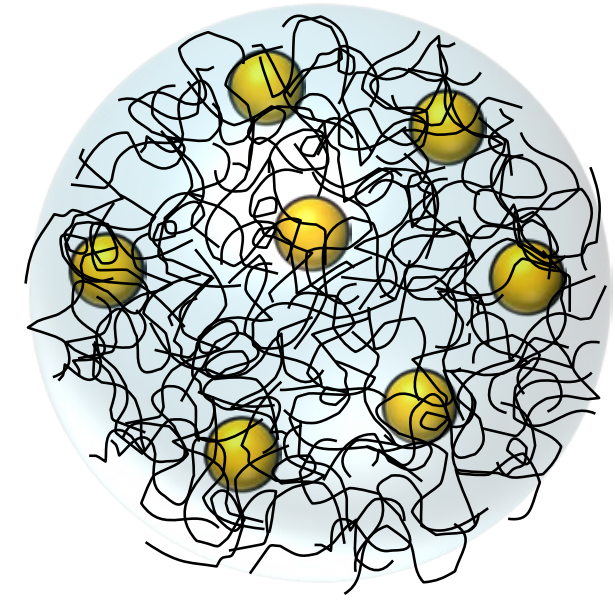

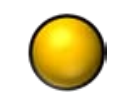

$\bigcirc$

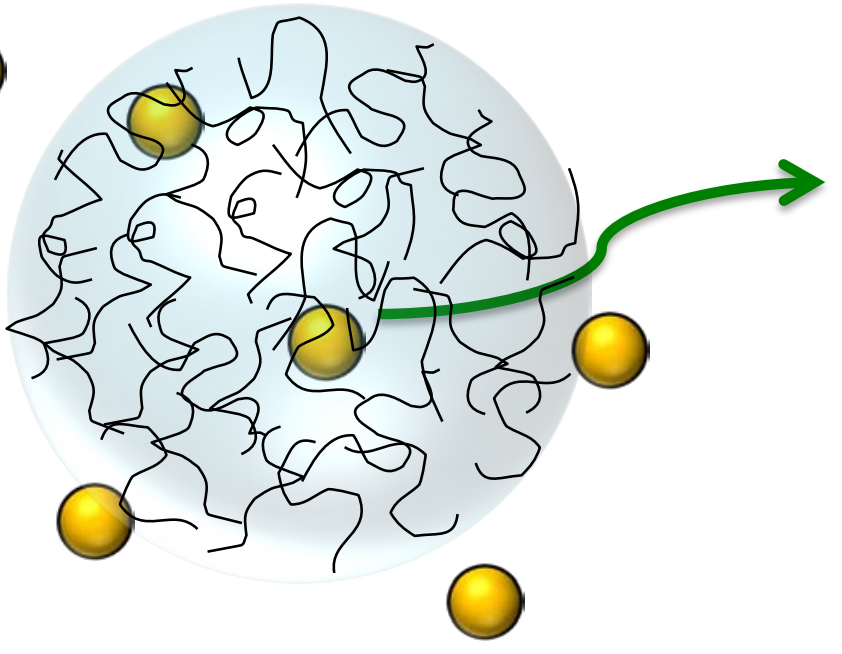


Figure 9: Schematic representation of the dependence of the lightness of microgel suspensions on the particle concentration, diameter, and relative refractive index.
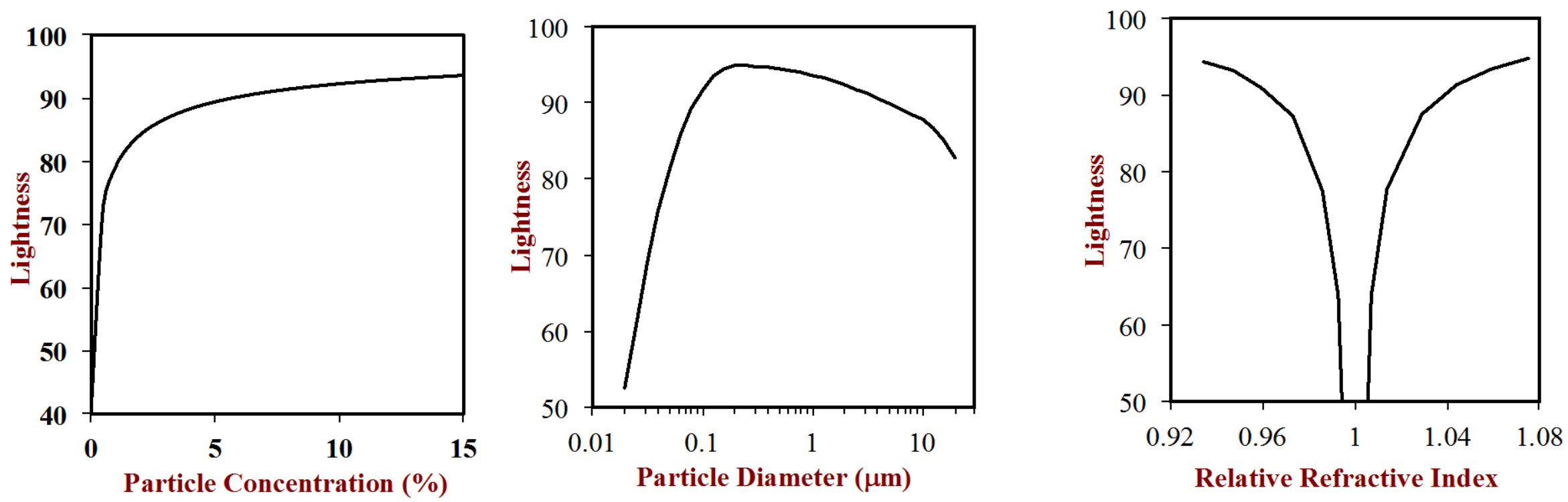
Figure 10: Gravitational sedimentation in microgel suspensions can be controlled by incorporating lipid droplets within the microgel particles to alter the density contrast. The red arrow indicates a sediment of microgel particles at the bottom of the test tube.

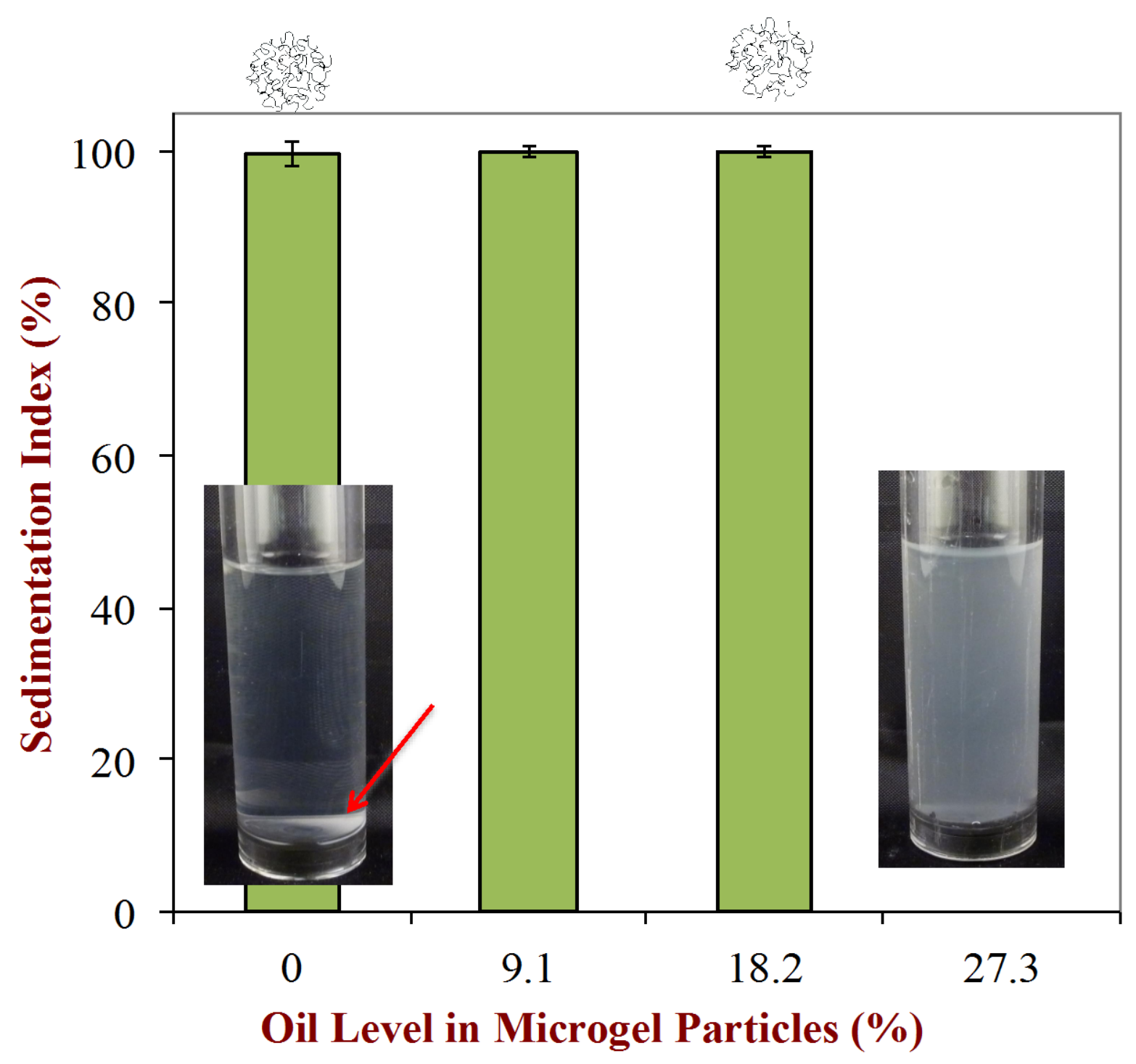


Figure 11: Predicted values of the van der Waals attraction, electrostatic repulsion, and steric repulsion between microgel beads ( $10 \mu \mathrm{m}$ radius)

\section{van der Waals attraction}

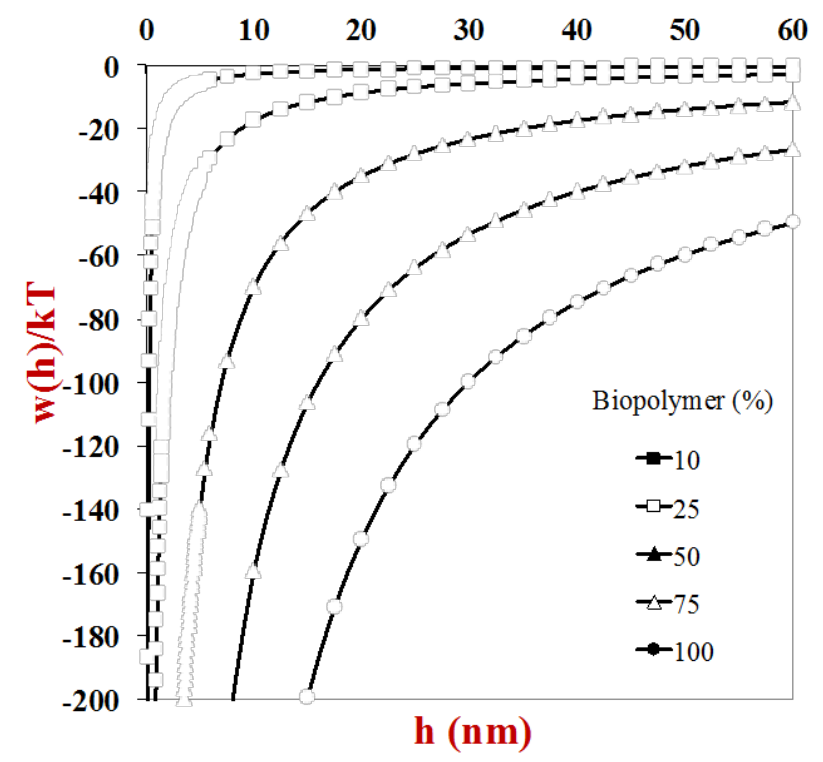

\section{Electrostatic repulsion}

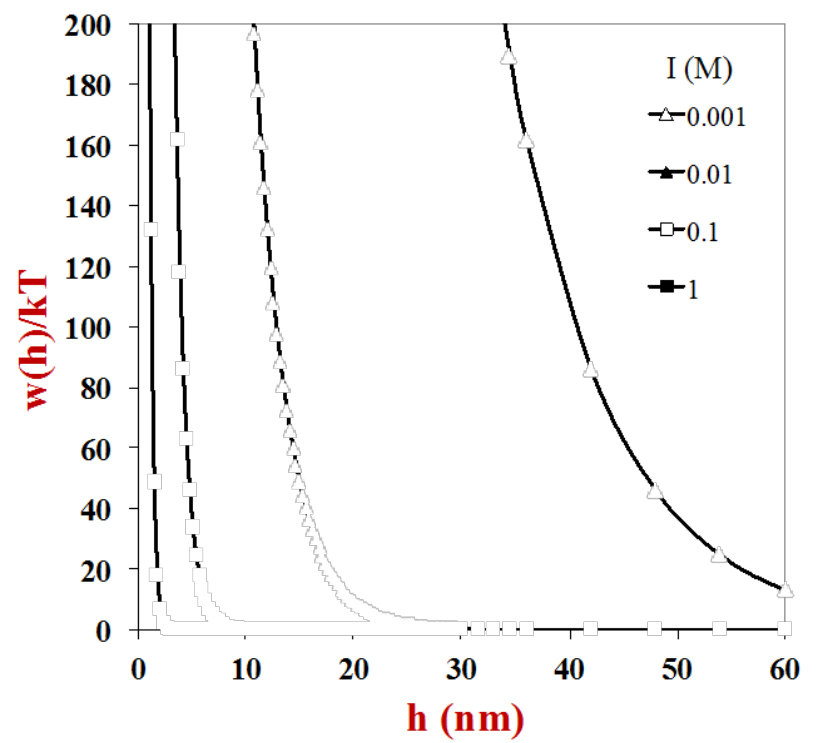

\section{Steric repulsion}

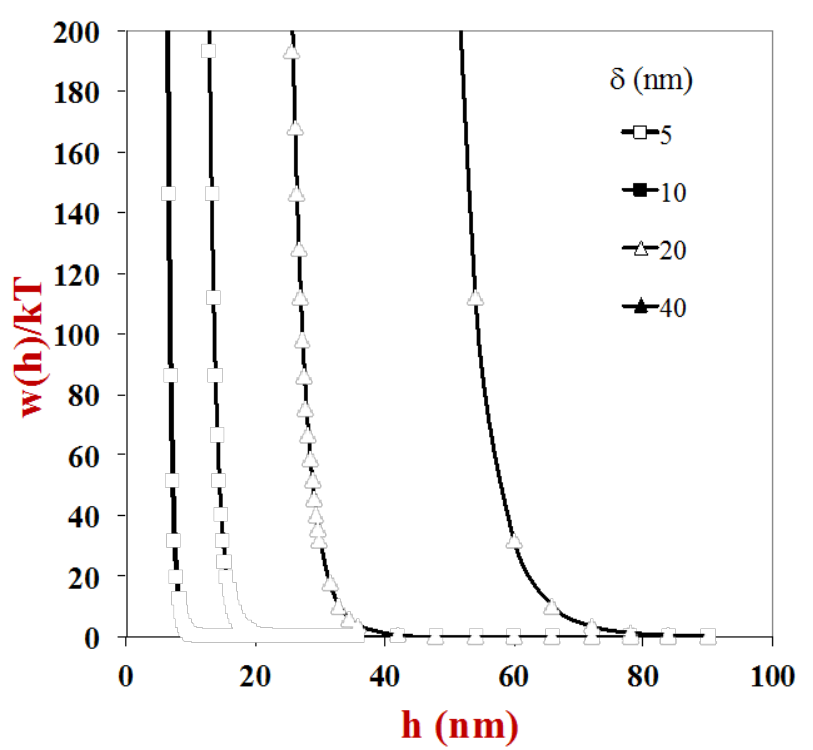


Figure 12: Predicted values of the van der Waals, electrostatic, steric and total interactions between microgel beads (10 $\mu$ m radius)

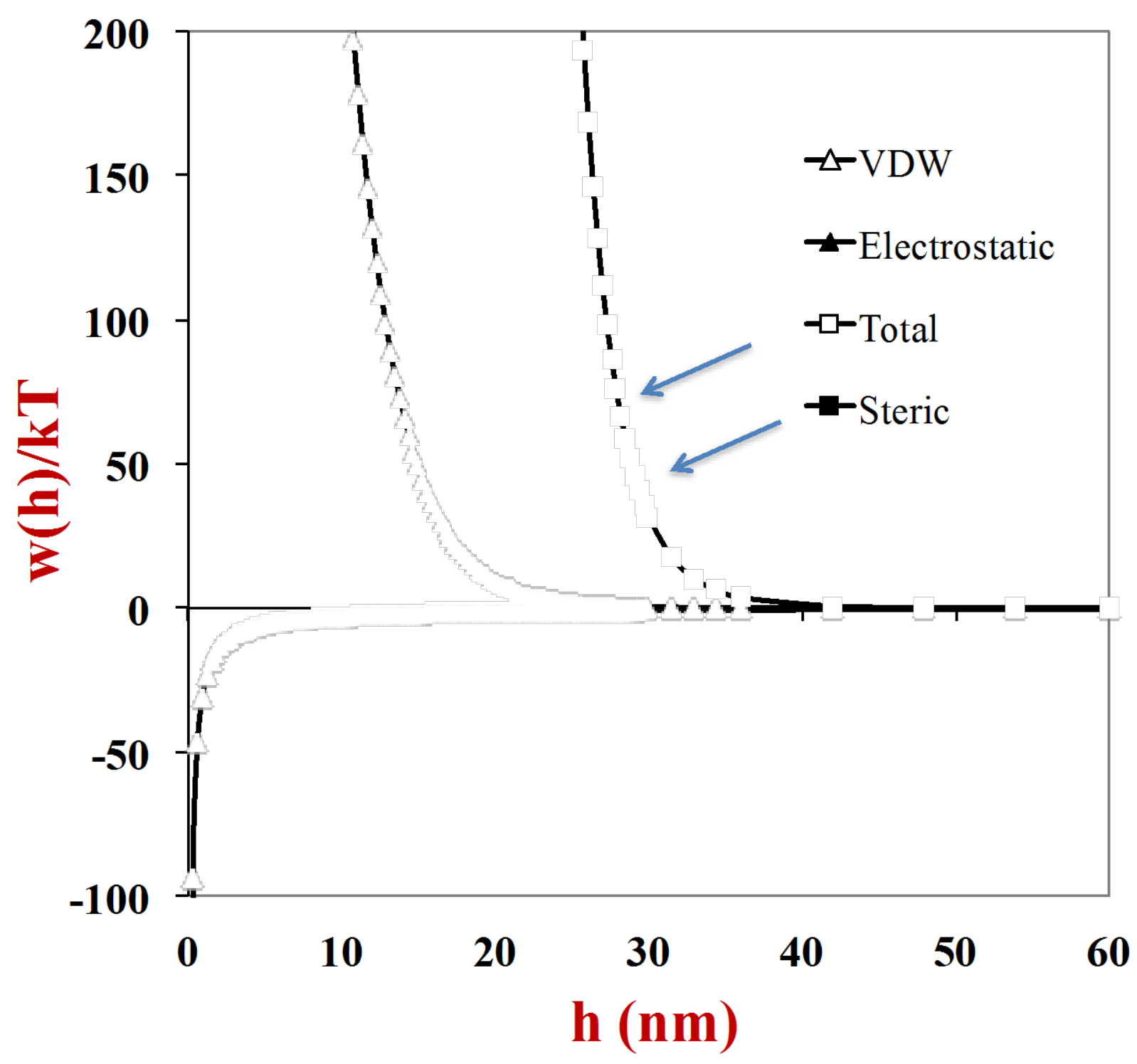


Figure 13: Dependence of the relative viscosity of microgel suspensions on the (a) effective volume fraction of microgel particles and (b) the total biopolymer concentration (for different volume ratios).

(a)

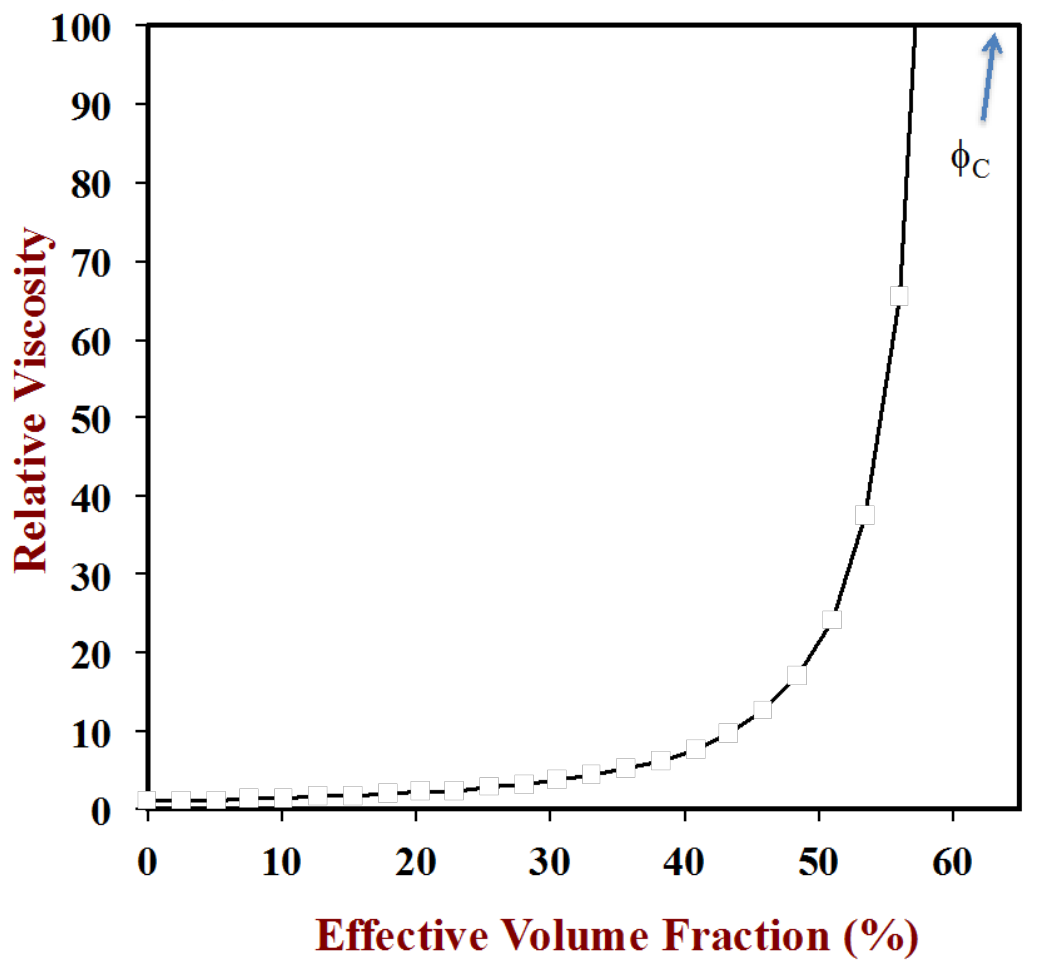

(b)

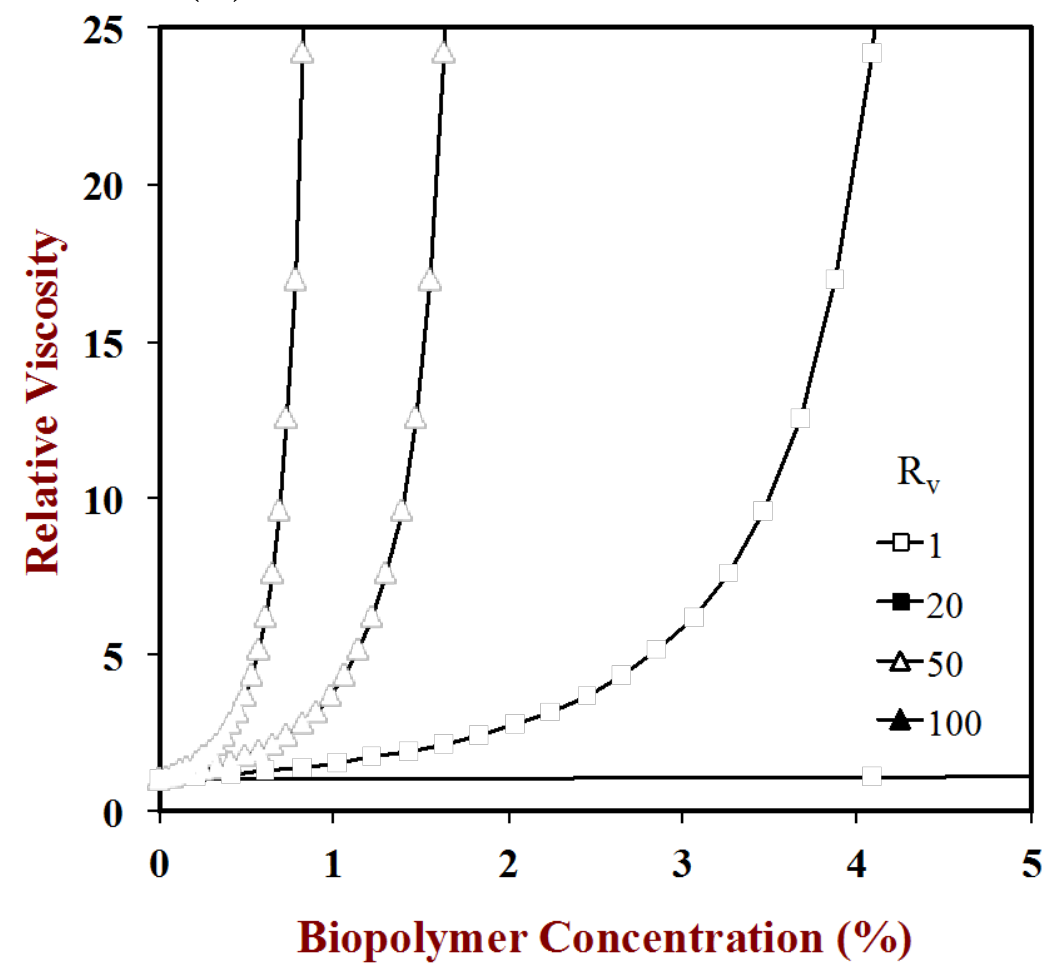


Figure 13: Dependence of the shear modulus and yield stress of non-food grade microgel suspensions on the amount of polyelectrolyte present. The modulus and yield stress increase with increasing polymer concentration above the critical packing concentration. Data from Pellet and Colitre (2016).

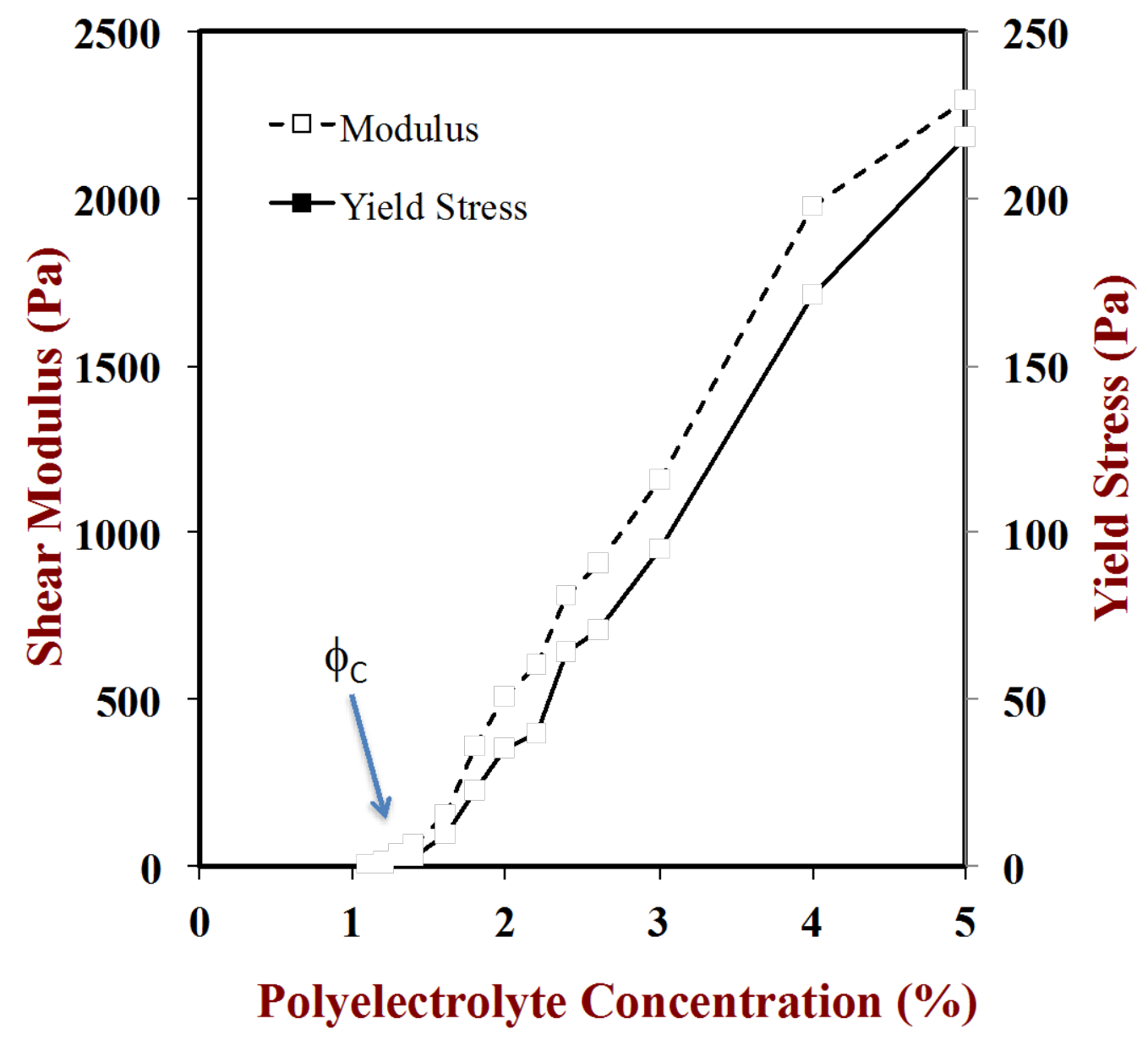


Figure 15a: Bioactive agents (molecules or particles) may be released from microgel particles through a number of physicochemical mechanisms, such as diffusion, swelling, or disintegration (erosion or fragmentation)

\section{Simple Diffusion}

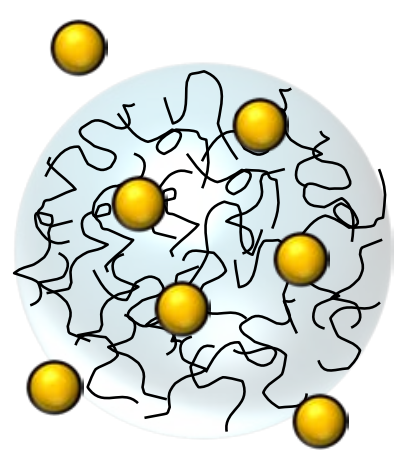

\section{Swelling}

\&

Diffusion

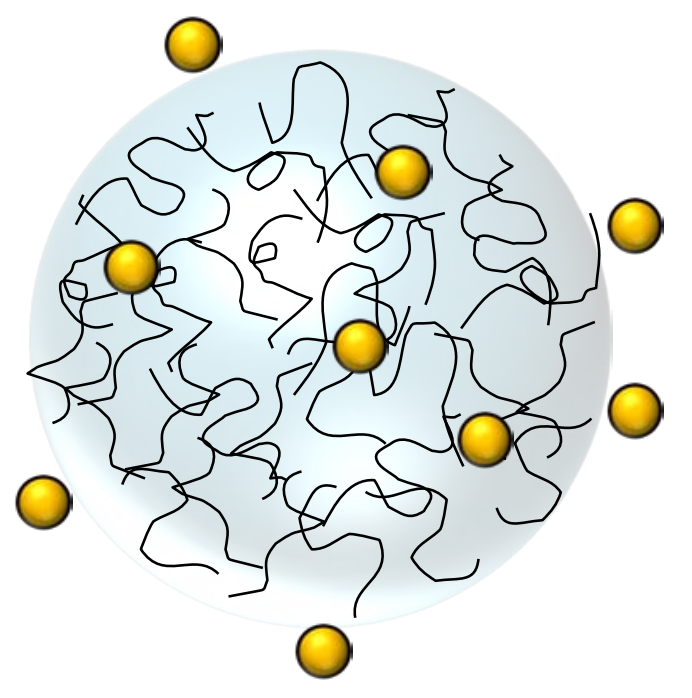

\section{Disintegration}

\&

Diffusion

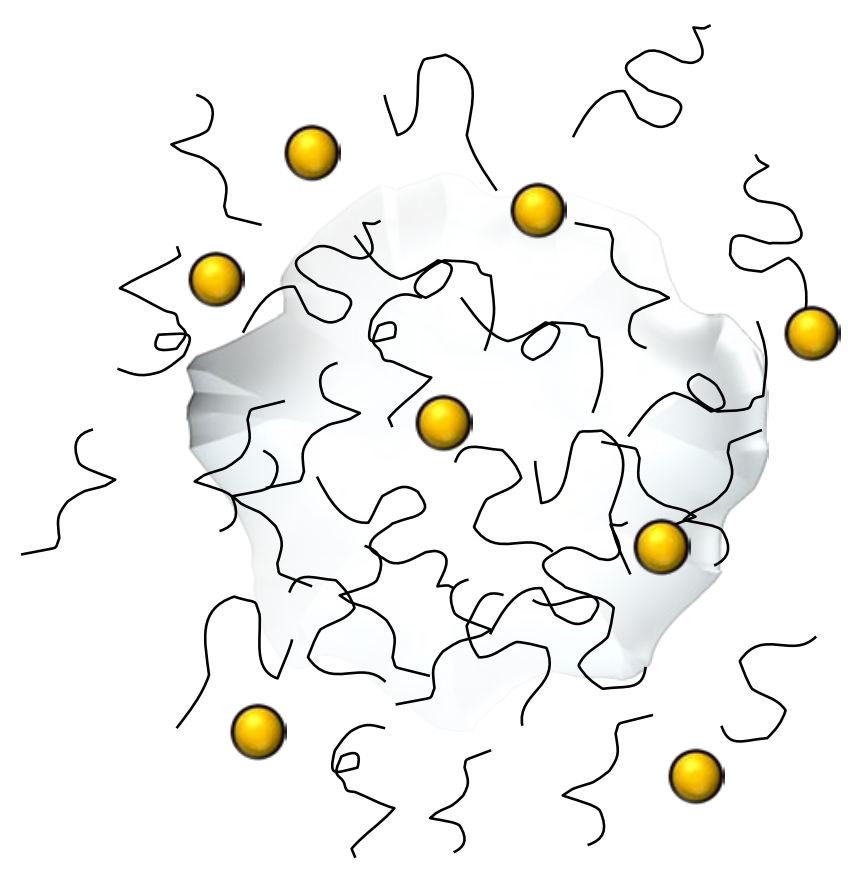


Figure 15b: Bioactive agents are retained when there is a strong enough attraction to the biopolymer network in the microgel particles, but released otherwise.
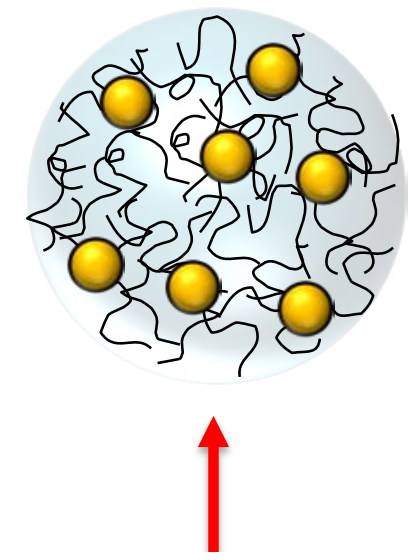

Retention

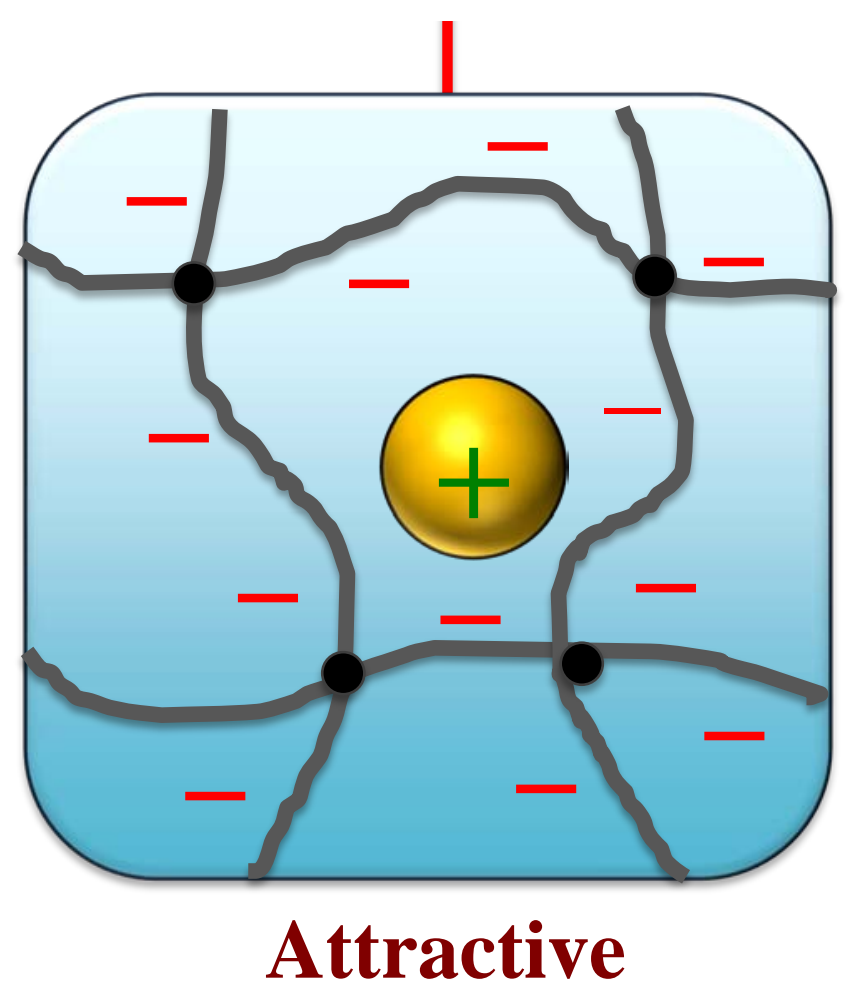

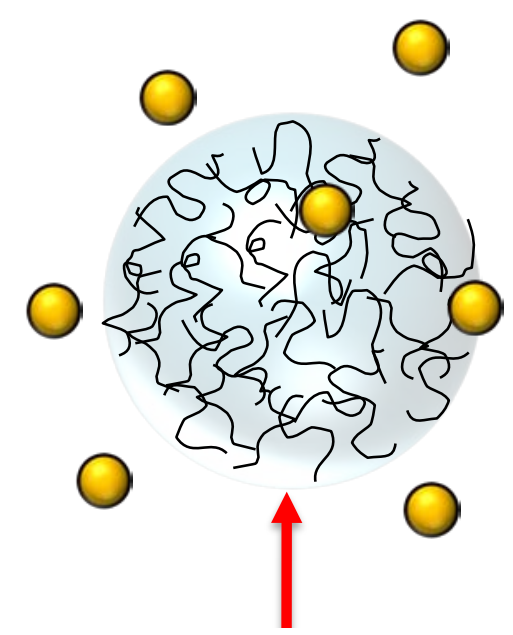

Release

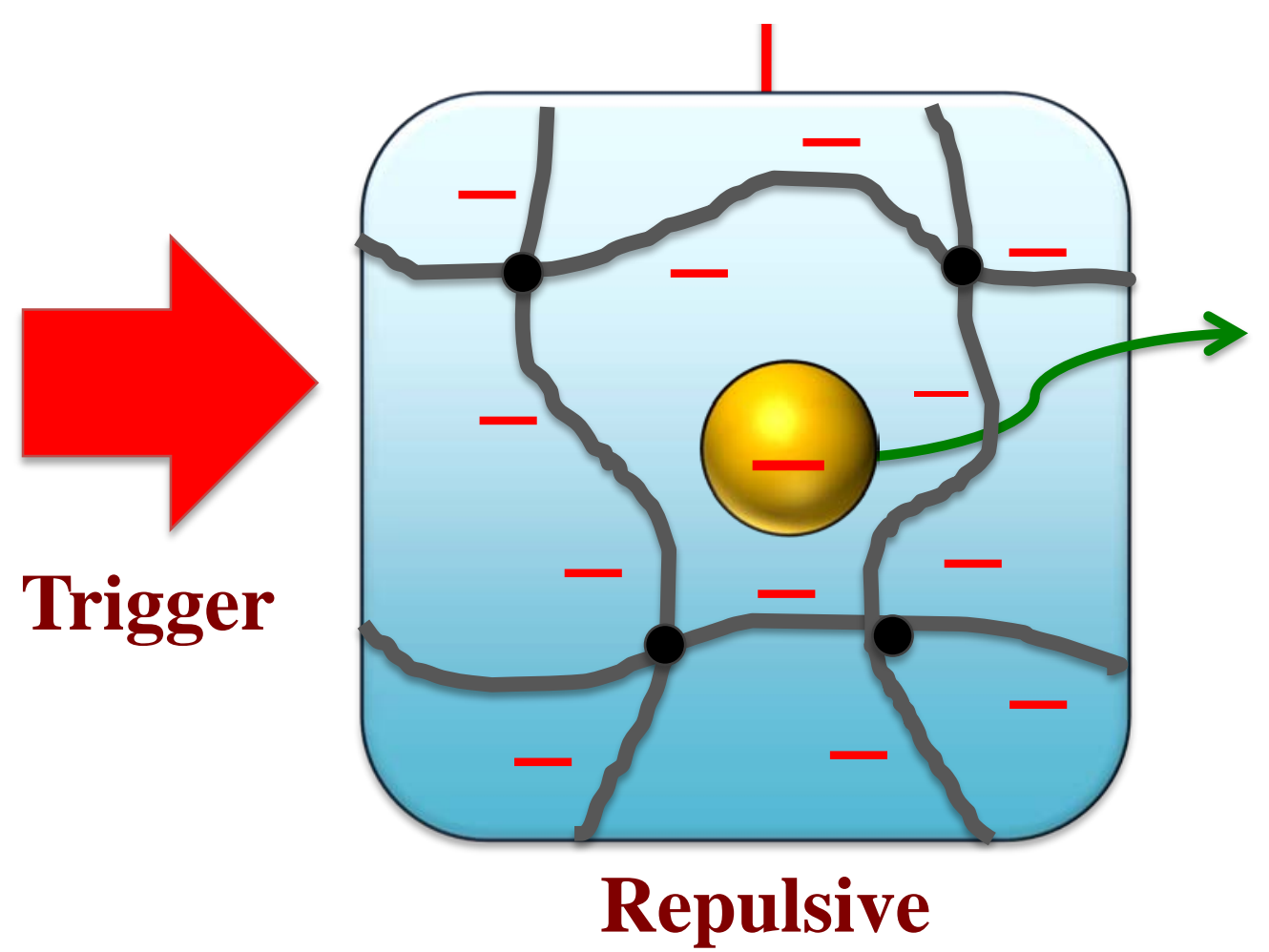


Figure 16: Predicted influence of the release kinetics of bioactive molecules from microgel particles due to simple diffusion. The release rate was calculated assuming a pore radius of $50 \mathrm{~nm}$ and a bioactive radius of 50 nm.

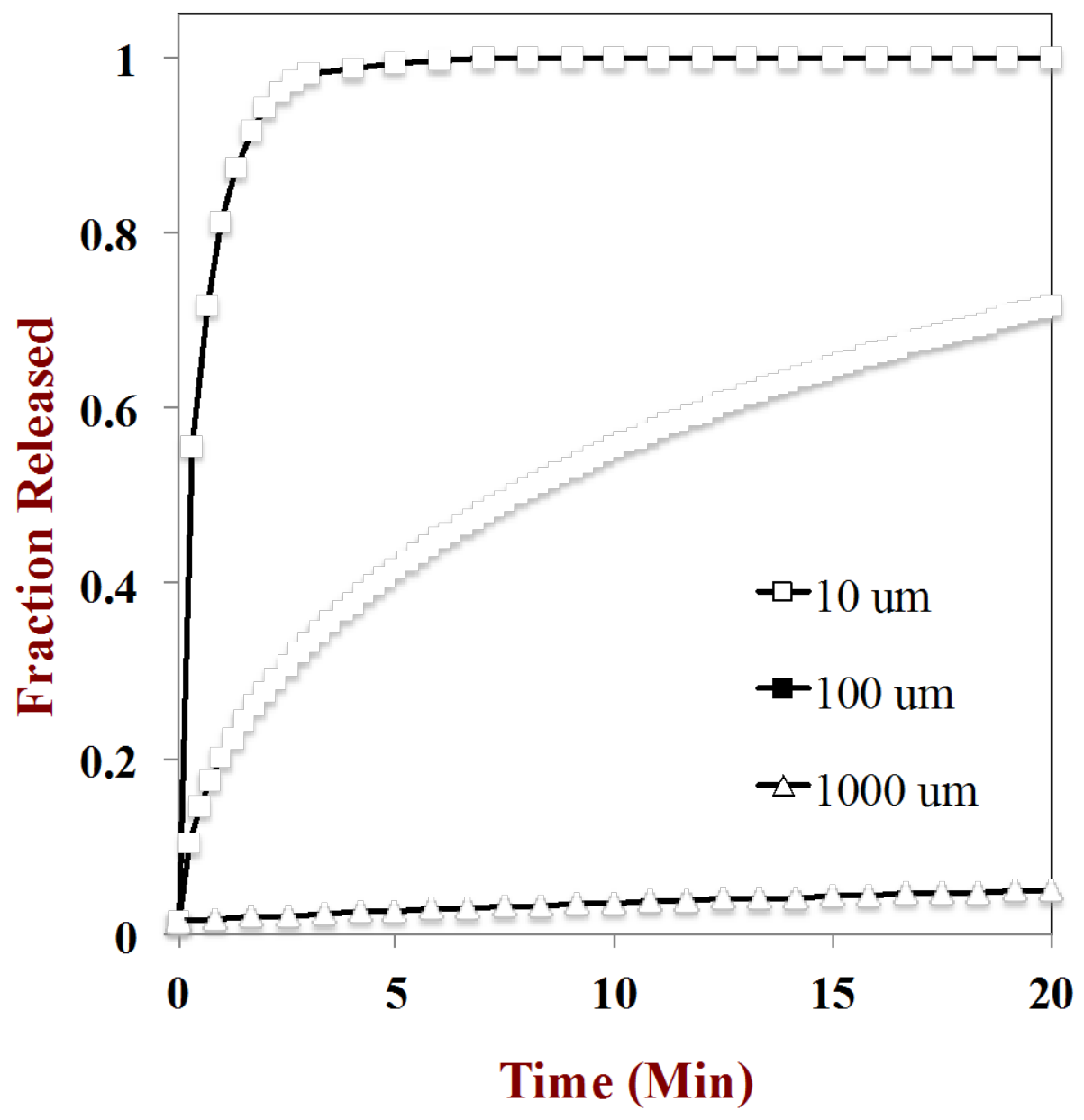


Figure 17a: Predicted influence of the release kinetics of bioactive molecules from microgel particles due to simple diffusion. The release rate was calculated assuming a bioactive radius of $50 \mathrm{~nm}$ and a microgel particle diameter of $100 \mu \mathrm{m}$.

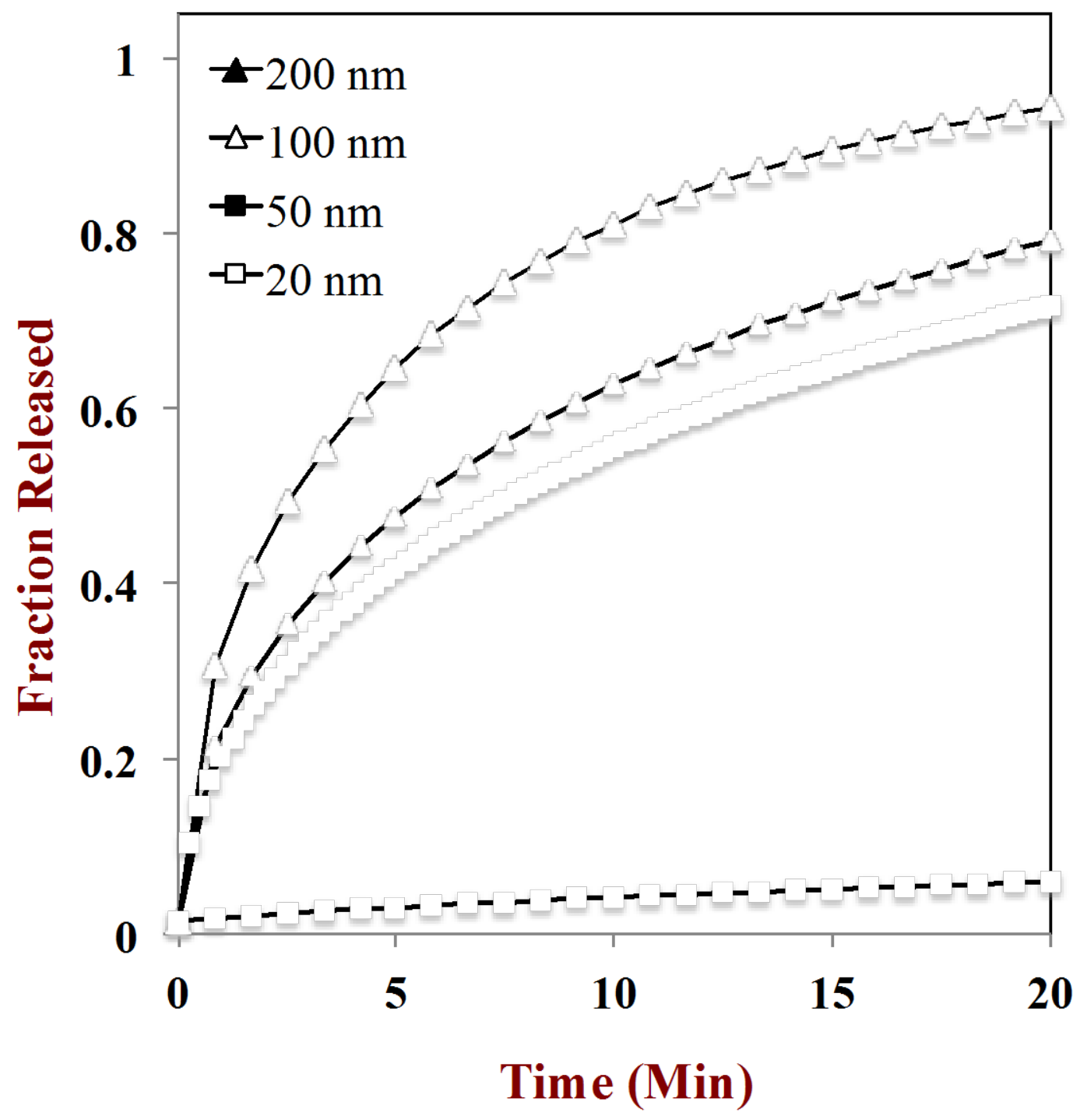


Figure 17b: Predicted impact of the pore diameter on relative diffusion coefficient of bioactive molecules through a biopolymer network with different pore diameters. The release rate was calculated for bioactive agents with different radii $(1,10,100$ and $1000 \mathrm{~nm})$.

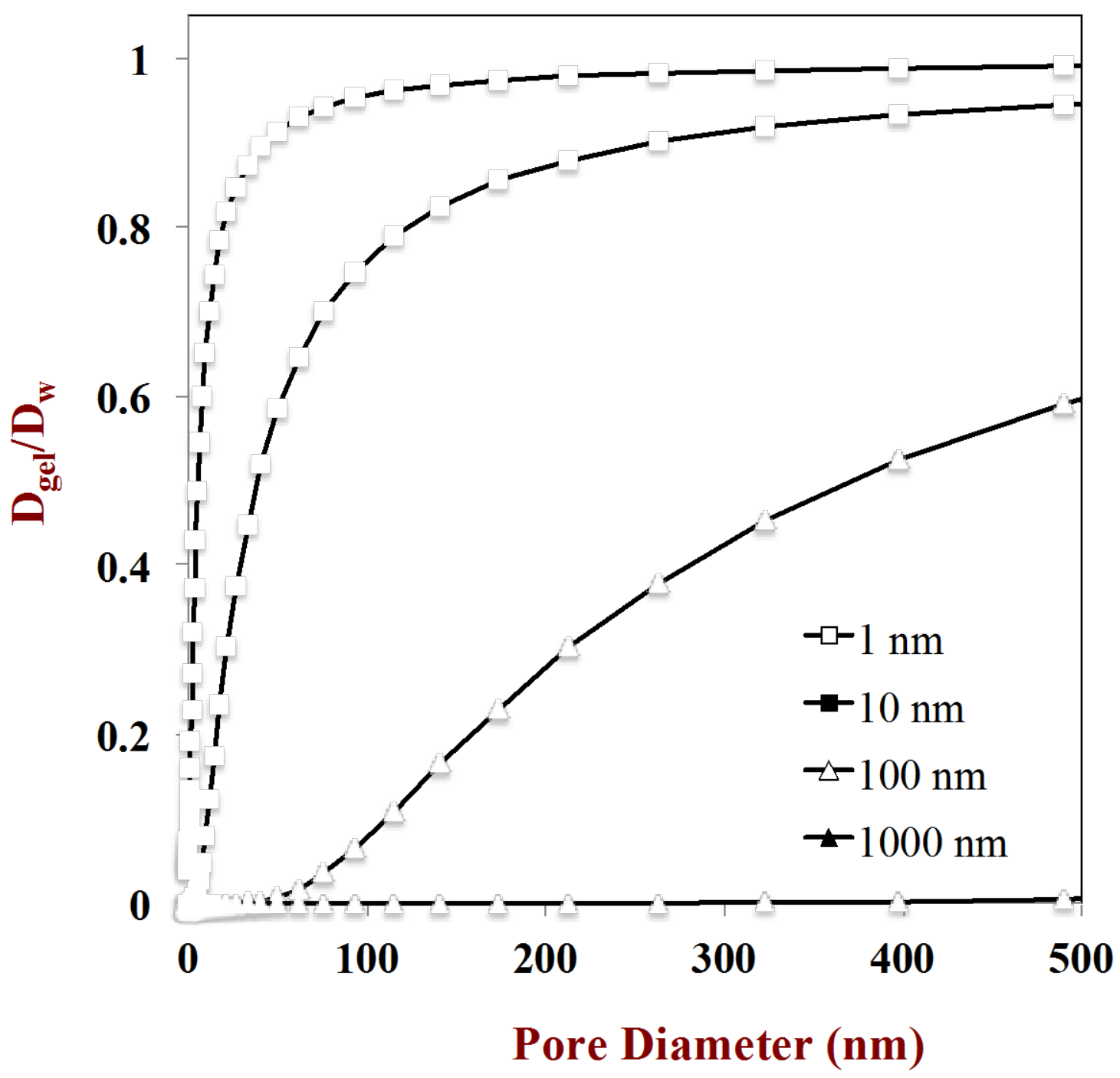


Figure 17c: The release of curcumin-loaded lipid droplets from alginate microgels (after 24 hour storage) decreased with increasing alginate concentration, which was attributed to a decrease in pore size (Zeeb et al 2015).

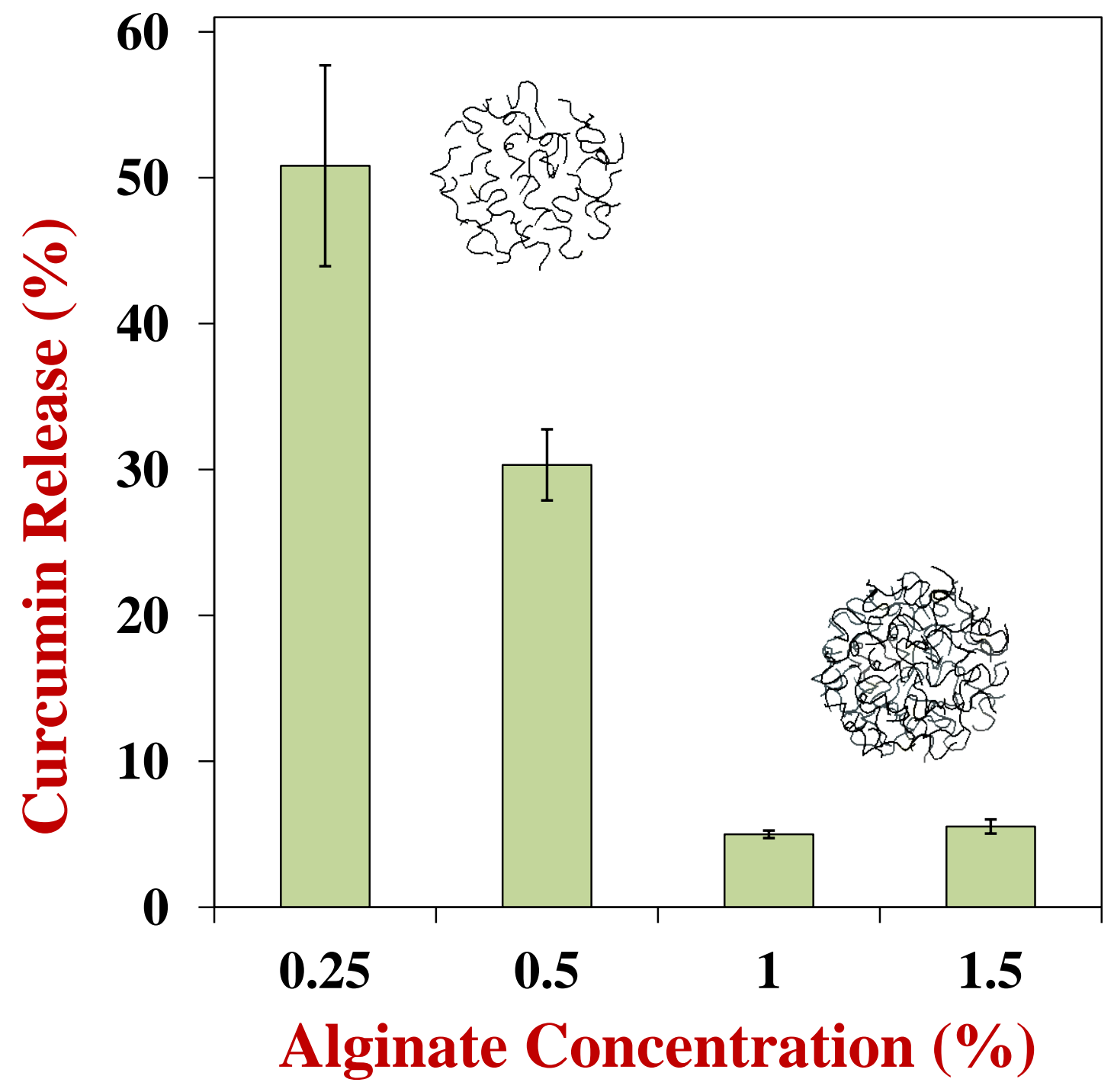


Figure 18: The impact of preparation $\mathrm{pH}$ on the retention of protein in calcium alginate beads (Zhang et al 2016). The amount of protein encapsulated decreased with increasing $\mathrm{pH}$ due to weakening of electrostatic attraction between the protein molecules and alginate in the hydrogel matrix (Zhang et al 2016).

(a)

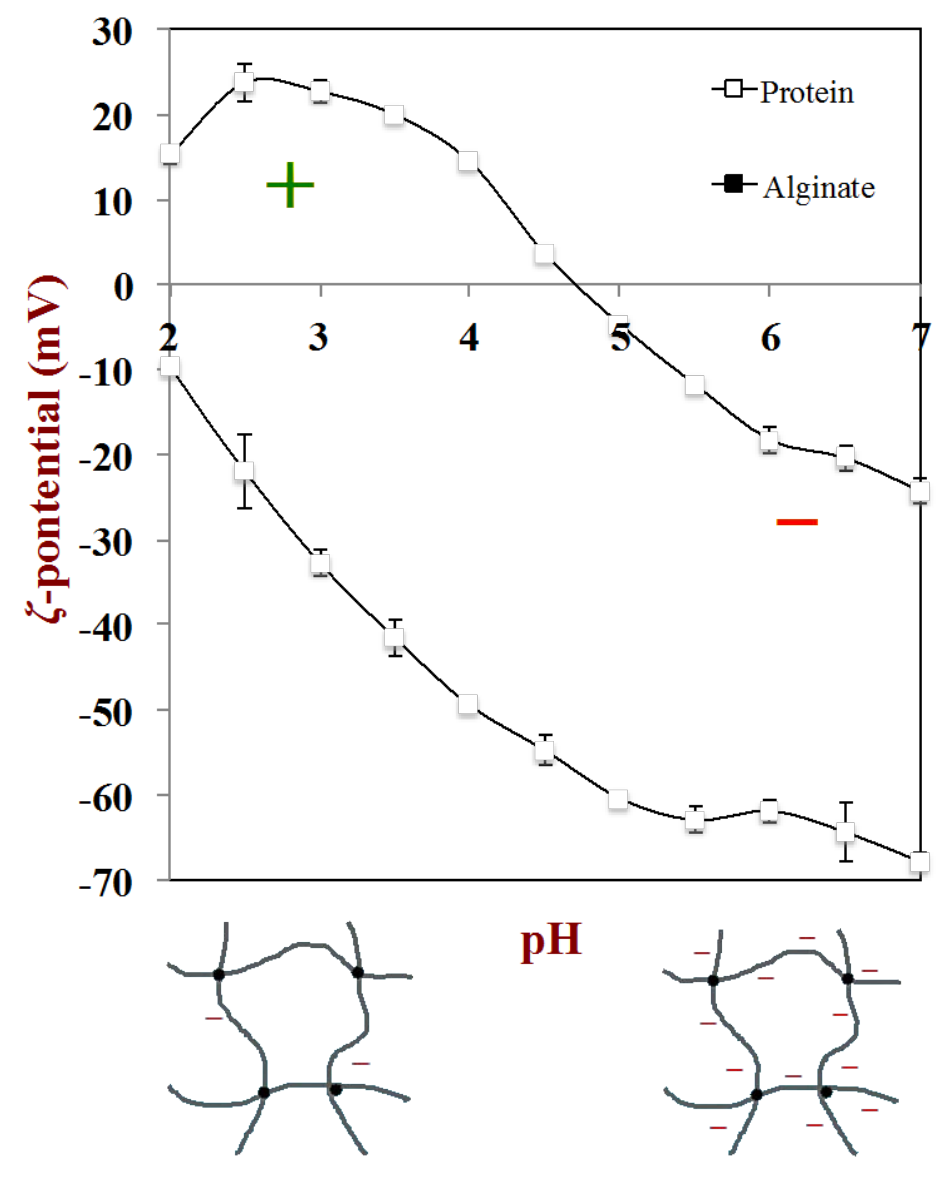

(b)

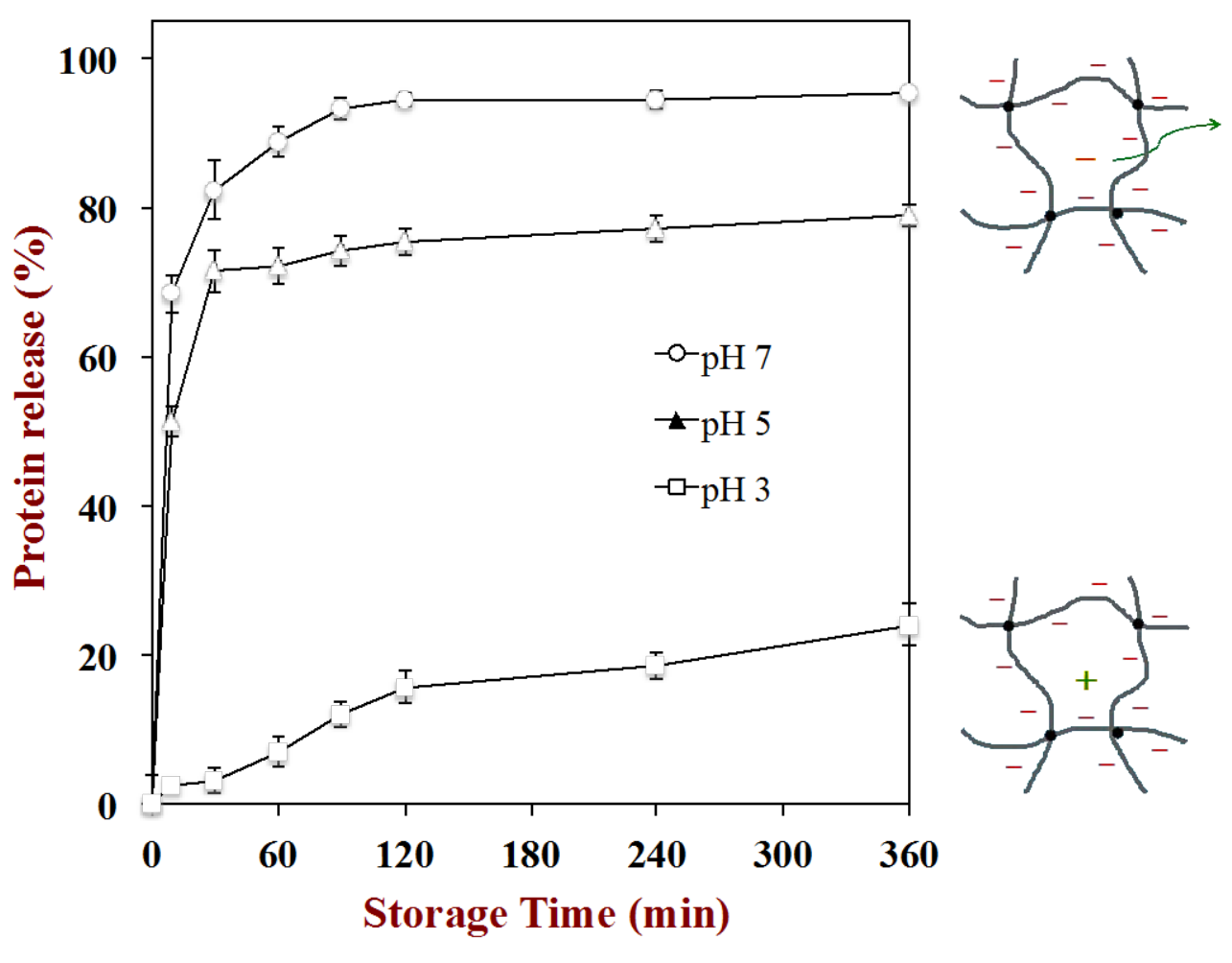


Figure 19: Bioactive agents can be released in different regions of the GIT by selecting biopolymers with different sensitivities to gastrointestinal conditions, such as $\mathrm{pH}$, ionic strength, or enzyme activity.

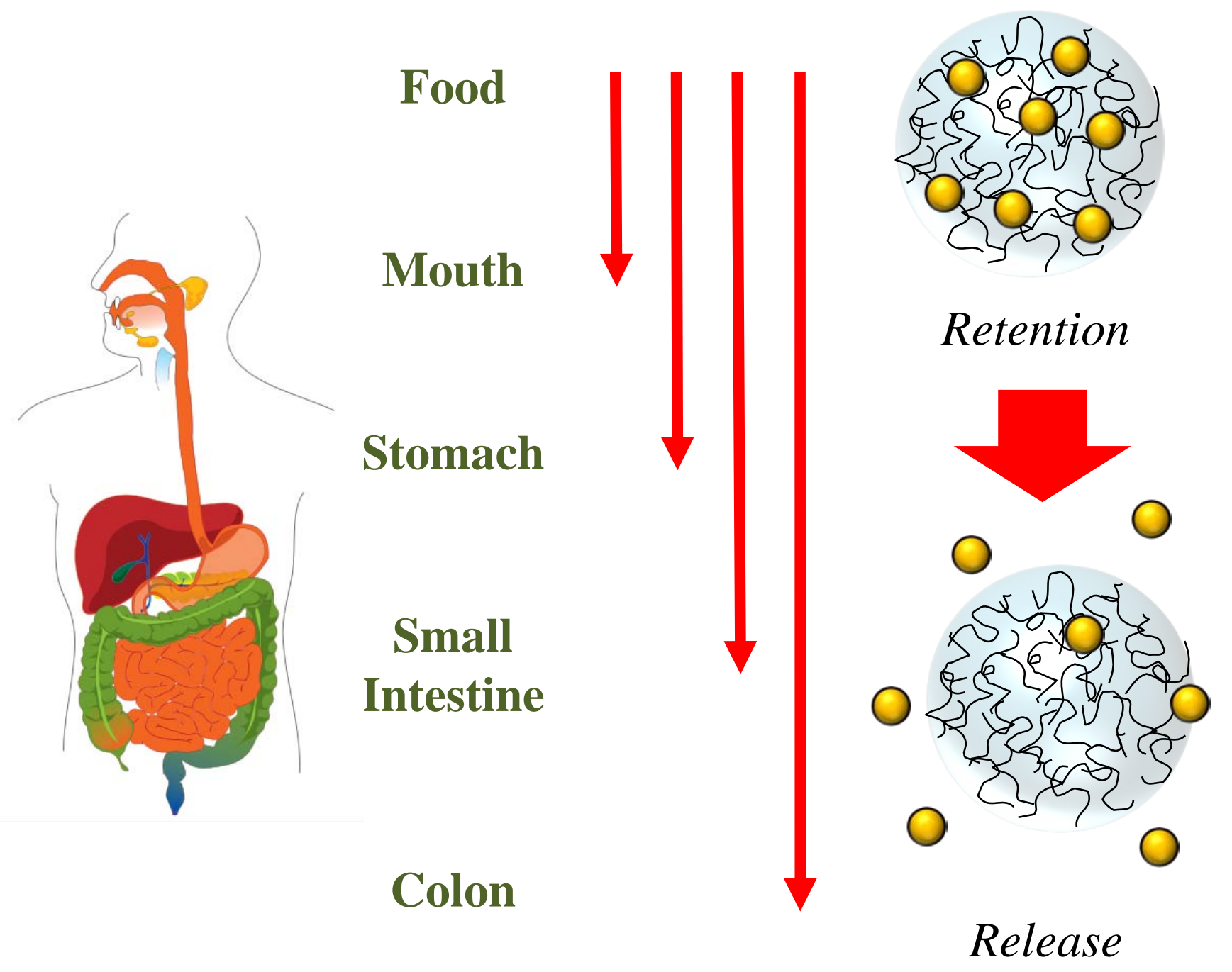


Figure 20: Schematic representation of some common types of release profile that might be utilized for bioactive agents encapsulated in microgel particles.

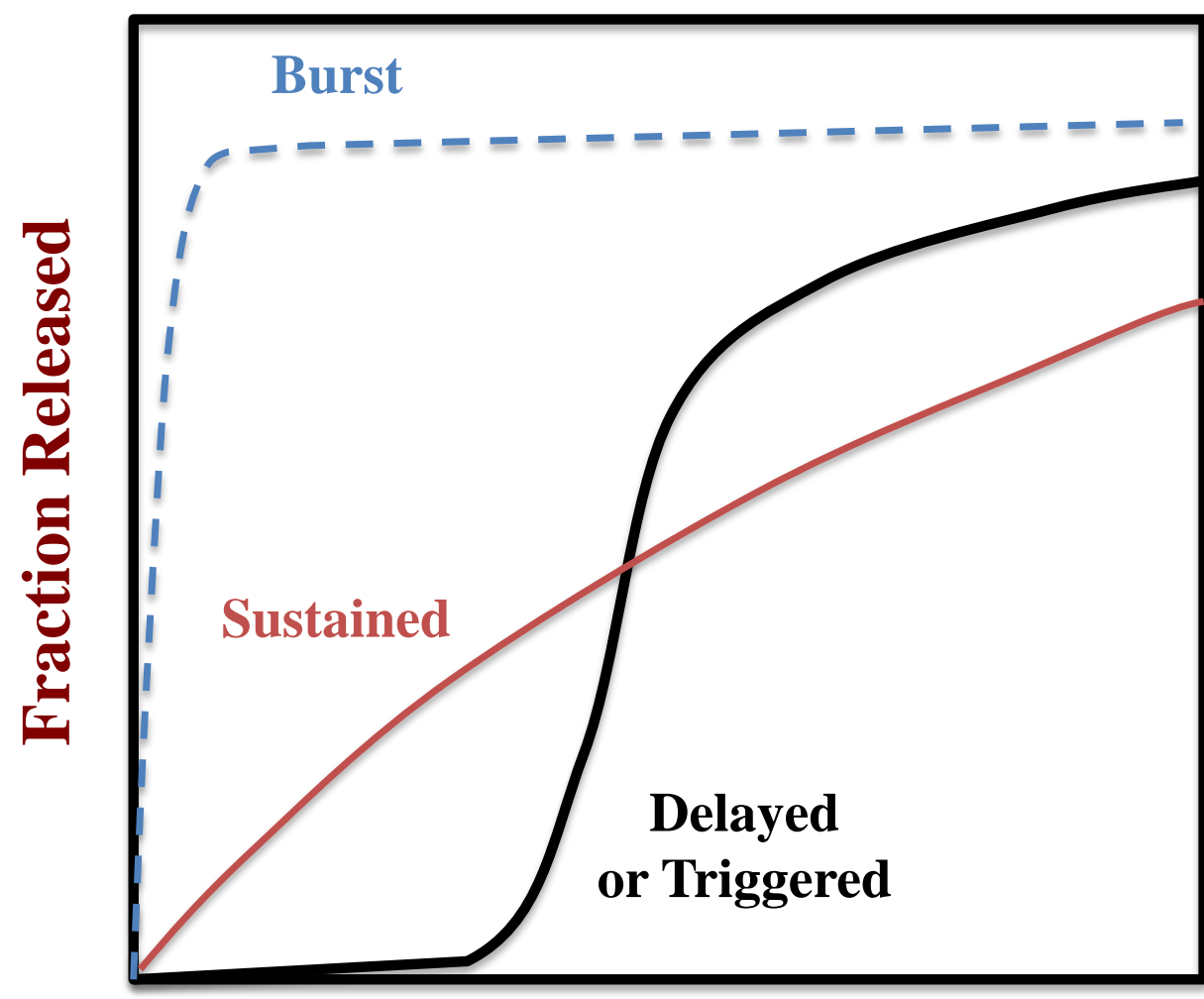

Release Time 
Figure 21: Fluorescence intensity inside alginate microgel particles (in the presence and absence of co-encapsulated magnesium hydroxide) after exposure to simulated gastric conditions. In the presence of buffer the $\mathrm{pH}$ inside the particles remains close to neutral (high intensity), while in the absence of buffer the $\mathrm{pH}$ is highly acidic (low intensity). 
Table 1a. Overview of some of the molecular characteristics of selected food-grade proteins that can be used to fabricate microgels. Here, $\mathrm{pI}$ is the isoelectric point and $T_{\mathrm{m}}$ is the thermal transition temperature. Key: A: Type A Gelatin; B: Type B Gelatin; S: S-Type Ovalbumin; 7S and 11S Soy Glycinin fractions.

\begin{tabular}{|c|c|c|c|c|c|}
\hline Name & Main Source & $\begin{array}{c}\text { Main } \\
\text { Structural } \\
\text { Type }\end{array}$ & pI & $\sim \mathbf{T}_{\mathbf{m}}\left({ }^{\circ} \mathbf{C}\right)$ & Solubility \\
\hline $\begin{array}{l}\text { Whey protein } \\
\text { ( } \beta \text {-lactoglobulin) }\end{array}$ & Milk & Globular & $\sim 5.0$ & $\sim 75$ & Water \\
\hline Caseins & Milk & Flexible & $\sim 4.6$ & $\sim 125-140$ & Water \\
\hline $\begin{array}{l}\text { Bovine Serum } \\
\text { Albumin }\end{array}$ & Milk/Blood & Globular & $\sim 4.7$ & $\sim 80$ & Water \\
\hline Lactoferrin & Milk & Globular & $\sim 8.0$ & $\sim 60 \& 90$ & Water \\
\hline Ovalbumin & Egg White & Globular & $\sim 4.6$ & $\sim 74 ; 82^{\mathrm{S}}$ & Water \\
\hline Lysozyme & Egg White & Globular & $\sim 11.0$ & $\sim 74$ & Water \\
\hline Phosvitin & Egg Yolk & Globular & $\sim 4.0$ & $\sim 80$ & Water \\
\hline Gelatin & $\begin{array}{c}\text { Animal or } \\
\text { Fish Collagen }\end{array}$ & Flexible & $\begin{array}{l}\sim 8^{\mathrm{A}} \\
\sim 5^{\mathrm{B}}\end{array}$ & $\begin{aligned} & \sim 5 \text { (fish) } \\
\sim & 40(\text { animal })\end{aligned}$ & Water \\
\hline Soy Glycinin & Soybean & Globular & $\sim 5.0$ & $\sim 67^{7 \mathrm{~S}} ; 87^{11 \mathrm{~S}}$ & Water \\
\hline Glutelin & Rice & Globular & $\sim 4.0$ & $\sim 82$ & Water \\
\hline Zein & Corn & Globular & $\sim 6$ & $\sim 90$ & $\begin{array}{l}\text { Organic } \\
\text { Solvent }\end{array}$ \\
\hline Gliadin & Wheat & Globular & $\sim 4.8$ & $\sim 47$ & $\begin{array}{l}\text { Organic } \\
\text { Solvent }\end{array}$ \\
\hline
\end{tabular}


Table 1b. Overview of some of the molecular characteristics of selected food-grade polysaccharides that can be used to fabricate microgels.

\begin{tabular}{|c|c|c|c|c|}
\hline Name & Source & $\begin{array}{c}\text { Main } \\
\text { Structure } \\
\text { Type }\end{array}$ & $\begin{array}{l}\text { Charge/Polar } \\
\text { Characteristics }\end{array}$ & Gelation \\
\hline Agar & Algal & Linear & $\begin{array}{l}\text { Neutral } \\
\text { Polar }\end{array}$ & $\begin{array}{l}\text { Cold-set } \\
\text { (reversible) }\end{array}$ \\
\hline Alginate & Algal & Linear & $\begin{array}{l}\text { Anionic } \\
\text { Polar }\end{array}$ & $\begin{array}{c}\mathrm{Ca}^{2+} \\
\text { cross-linking }\end{array}$ \\
\hline Carrageenan & Algal & Linear/Helical & $\begin{array}{l}\text { Anionic } \\
\text { Polar } \\
\end{array}$ & $\begin{array}{c}\text { Cold Set } \\
\left(\mathrm{K}^{+} \text {or } \mathrm{Ca}^{2+}\right)\end{array}$ \\
\hline Chitosan & $\begin{array}{l}\text { Crustaceans, } \\
\text { Invertebrates }\end{array}$ & Linear & $\begin{array}{l}\text { Cationic } \\
\text { Polar }\end{array}$ & $\begin{array}{l}\text { Polyphosphate } \\
\text { Cross-linking }\end{array}$ \\
\hline Gum Arabic & Acacia Sap & $\begin{array}{c}\text { Branched } \\
\text { Coil Domains } \\
\text { on Protein } \\
\text { Scaffold } \\
\end{array}$ & $\begin{array}{c}\text { Anionic } \\
\text { Polar } \\
\text { Amphiphilic }\end{array}$ & $\begin{array}{l}\text { Forms gels at high } \\
\text { concentration }\end{array}$ \\
\hline Inulin & $\begin{array}{l}\text { Plants or } \\
\text { Bacteria }\end{array}$ & $\begin{array}{c}\text { Linear with } \\
\text { occasional } \\
\text { branches }\end{array}$ & $\begin{array}{l}\text { Neutral } \\
\text { Polar }\end{array}$ & $\begin{array}{l}\text { Forms gels at high } \\
\text { concentration }\end{array}$ \\
\hline $\begin{array}{c}\text { Methyl } \\
\text { Cellulose }\end{array}$ & Wood Pulp & Linear & Neutral & $\begin{array}{c}\text { Heat-Set } \\
\text { (reversible) }\end{array}$ \\
\hline $\begin{array}{l}\text { Pectin } \\
\text { (Fruit) }\end{array}$ & $\begin{array}{l}\text { Plant Cell } \\
\text { Walls }\end{array}$ & $\begin{array}{c}\text { Highly } \\
\text { Branched } \\
\text { Coil } \\
\end{array}$ & $\begin{array}{l}\text { Anionic } \\
\text { Polar }\end{array}$ & $\begin{array}{l}\text { Sugar/Heat (HM); } \\
\qquad \mathrm{Ca}^{2+}(\mathrm{LM})\end{array}$ \\
\hline $\begin{array}{l}\text { Pectin } \\
\text { (Beet) }\end{array}$ & $\begin{array}{l}\text { Sugar Beet } \\
\text { Pulp }\end{array}$ & $\begin{array}{l}\text { Branched } \\
\text { Coil with } \\
\text { Protein }\end{array}$ & $\begin{array}{c}\text { Anionic } \\
\text { Polar } \\
\text { Amphiphilic }\end{array}$ & $\begin{array}{c}\text { Sugar/Heat }(\mathrm{HM}) \\
\mathrm{Ca}^{2+}(\mathrm{LM}) \\
\text { Laccase }\end{array}$ \\
\hline $\begin{array}{l}\text { Xanthan } \\
\text { Gum }\end{array}$ & Bacterial & $\begin{array}{l}\text { Linear/Helical } \\
\text { (High MW) }\end{array}$ & Anionic & $\begin{array}{l}\text { Forms gels at high } \\
\text { concentration }\end{array}$ \\
\hline
\end{tabular}

Note: Commercially available polysaccharide ingredients typically contain appreciably different molecular and functional properties; the listed information describes general characteristics for industrial usage. 
Table 2. Predicted sedimentation stability of microgel particles with different compositions (biopolymer levels) and sizes dispersed in water.

\begin{tabular}{|c|c|c|c|c|c|}
\hline & & \multicolumn{4}{|c|}{ Sedimentation Velocity (mm/day) } \\
\hline $\begin{array}{c}\text { Biopolymer } \\
\text { Level }(\mathrm{v} / \mathrm{v} \%)\end{array}$ & $\begin{array}{c}\text { Density } \\
\left(\mathrm{kg} / \mathrm{m}^{3}\right)\end{array}$ & $\mathrm{D}=0.1 \mu \mathrm{m}$ & $\mathrm{D}=1 \mu \mathrm{m}$ & $\mathrm{D}=10 \mu \mathrm{m}$ & $\mathrm{D}=100 \mu \mathrm{m}$ \\
\hline 1 & 1006 & 0.0026 & 0.26 & 26 & 2,600 \\
\hline 2 & 1012 & 0.0052 & 0.52 & 52 & 5,200 \\
\hline 5 & 1030 & 0.013 & 1.3 & 130 & 13,000 \\
\hline 10 & 1060 & 0.026 & 2.6 & 260 & 26,000 \\
\hline 20 & 1120 & 0.052 & 5.2 & 520 & 52,000 \\
\hline 50 & 1300 & 0.13 & 13 & 1300 & 130,000 \\
\hline 100 & 1600 & 0.26 & 26 & 2600 & 260,000 \\
\hline
\end{tabular}




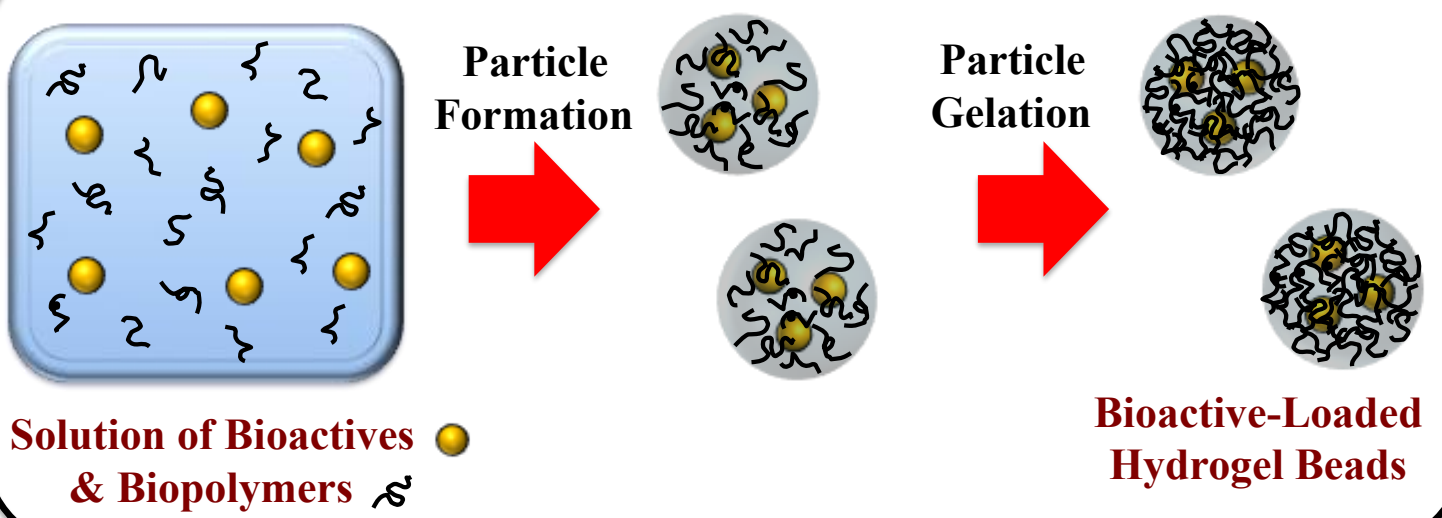

